\title{
Acknowledgment to Reviewers of Cancers in 2021
}

\author{
Cancers Editorial Office
}

Citation: Cancers Editorial Office. Acknowledgment to Reviewers of Cancers in 2021. Cancers 2022, 14, 734. https://doi.org/10.3390/

cancers14030734

Published: 30 January 2022

Publisher's Note: MDPI stays neutral with regard to jurisdictional claims in published maps and institutional affiliations.

\section{(i)}

Copyright: (C) 2022 by the author. Licensee MDPI, Basel, Switzerland. This article is an open access article distributed under the terms and conditions of the Creative Commons Attribution (CC BY) license (https://creativecommons.org/licenses/by/4.0/).

MDPI AG, St. Alban-Anlage 66, 4052 Basel, Switzerland

Rigorous peer-reviews are the basis of high-quality academic publishing. Thanks to the great efforts of our reviewers, Cancers was able to maintain its standards for the high quality of its published papers. Thanks to the contribution of our reviewers, in 2021, the median time to first decision was 18.5 days and the median time to publication was 40 days. The editors would like to extend their gratitude and recognition to the following reviewers for their precious time and dedication, regardless of whether the papers they reviewed were finally published:

Abal, Miguel

Abate, Giulia

Abbara, Ali

Abboud, Tammam

Abdović, Slaven

Abe, Tatsuya

Abed, Hasan

Abenavoli, Ludovico

Ablain, Julien

Aboubakar, Frank

Abraham, Dietmar

Abrantes, Ana Margarida

Abu Eid, Rasha

Abuhelwa, Ahmad

Abuja, Peter

Abusamra, Dina

Aceves, Carmen

Achreja, Abhinav

Acker, Güliz

Acquaviva, Claire

Acri, Giuseppe

Acs, Balazs

Adamczewski, Zbigniew

Adamczyk-Grochala, Jagoda

Adami, Guy R.

Adams, Brian D.

Adams, Tayloria

Adamska, Aleksandra

Adekoya, Timothy O.

Adhikari, Manish

Adighibe, Omanma

Aditya, Anusha

Adukauskienè, Dalia

Adunyah, Samuel E.
Afelik, Solomon

Afonso, Philippe

Afonso, Ricardo

Agarwal, Neeraj

Agarwal, Supreet

Agbarya, Abed

Ager, Ann

Agerbæk, Mette $\varnothing$.

Aggarwal, Vaishali

Agnieszka, Gizak

Agnoletto, Chiara

Agostinelli, Claudio

Agostini, Francesco

Agostini, Marco

Agresta, Ferdinando

Aguayo, Francisco

Agudo, Judith

Aguilar-Company, Juan

Aguilera, Nadine S.

Ahluwalia, Amrita

Ahluwalia, Pankaj

Ahmad, Ali

Ahmad, Fahim

Ahmad, Javed

Ahmed, Khalil

Ahmed, Zeeshan

Ahn, Kwang

Ahn, Seong Beom

Aigner, Achim

Aithal, Abhijit

Akagi, Kiwamu

Akam, Eman A.

Akarca, Ayse U.

Akiba, Jun 
Akingboye, Akinfemi

Akos, Nagy

Al Moustafa, Ala-Eddin

Al-Abdulla, Ruba

Alaggio, Rita

Alaimo, Alessandro

Alakel, Nael

Alarcón Fortepiani, María Lourdes

Alavi, Abass

Albano, Emanuele

Albers, Peter

Albert, Grinshpun

Albini, Adriana

Alcantara, Marion

Alcindor, Thierry

Aldieri, Elisabetta

Aldrighetti, Luca Antonio M.

Al-Dujaili, Emad

Aleem, Eiman

Aleman, Berthe M. P.

Alesi, Lauren R.

Alessandro, Tafuri

Alexander, H. Denis

Alexandraki, Krystallenia

Alexandre, Jérôme

Alexandre, Joachim

Alexandrova, Elena

Alexa-Stratulat, Teodora

Alfieri, Sergio

Al-Ghabkari, Abdulhameed

Algohary, Ahmad

Ali, Aiman

Ali, Syed Azmal

Alic, Lejla

Alifano, Marco

Alimandi, Maurizio

Alkhateeb, Abedalrhman

Allard-Chamard, Hugues

Allegretti, Matteo

Allegrucci, Cinzia

Allen-Petersen, Brittany

Allison, Simon J.

Allott, Louis

Allouche, Michèle

Al-Mubarak, Bashayer R.

Alonso-Colmenar, Luis M.

Alonso-Gordoa, Teresa

Alphonse, Martin Prince

Al-Qurayshi, Zaid

Alsaab, Hashem

Alsadeq, Ameera
Al-Sawaf, Othman

Alsbeih, Ghazi

Alsop, Kathryn

Althouse, Andrew

Altman, Brian J.

Altomare, Deborah A.

Altomonte, Jennifer

Alunni-Fabbroni, Marianna

Alvarado-Kristensson, Maria

Alvarez Cubero, María Jesús

Álvarez-Mercado, Ana I.

Álvarez-Sola, Gloria

Álvaro, Tomás

Alves Da Costa, Cristine

Alves, Francisco

Alves, Marco G.

Alzhrani, Rami M.

Amantini, Consuelo

AmaraL, Teresa

Amat, Ramon

Ambriovich Ristov, Andreja

Ambros, Peter

Ambrosini, Valentina

Ambrosino, Fabrizio

Ambs, Stefan

Amelio, Ivano

Amicone, Laura

Amir, Sabet

Ammendola, Michele

Ammirati, Mario

Amornyotin, Somchai

Amoruso, Giuseppe

Amos, Richard A.

Amouroux, Marine

Amrani, Abdelaziz

Amrutkar, Manoj

Amzar, Daniela

Anand, Kartik

Ananiev, Julian

Anastasiadou, Eleni

Anatskaya, Olga V.

Andersen, Thomas Levin

Anderson, Nigel J.

Anderson, Robin L.

Andersson, Jan

Andersson, Mattias K.

Andersson, Yvette

Ando, Katsuyoshi

Ando, Koji

Andón, Fernando Torres

André, Görgens 
André, Nathalie

Andrea, Levi

Andreani, Cristina

Andreassi, Maria

Andree, Kiki

Andreetti, Claudio

Andreev, Dmitry E.

Andreou, Dimosthenis

Andrés-León, Eduardo

Andreucci, Elena

Andreuzzi, Eva

Andrew, Angeline S.

Andrews, Miles

Andria, Gregorio

Andrieu, Nadine

Andritsch, Elisabeth

Androutsopoulos, Georgios

Anel, Alberto

Angalakurthi, Siva Krishna

Angelini, Francesco

Angelova, Mihaela

Angelucci, Cristiana

Angioni, Roberta

Angulo, Javier

Anikin, Vladimir

Anile, Marco

Anken, Ralf

Annabi, Borhane

Annunziata, Christina Messineo

Ansa, Benjamin

Ansarin, Mohssen

Ansems, Marleen

Anthony, Donald

Antić, Željko

Antica, Mariastefania

Antón, Antonio

Antonelli, Alessandro

Antonelli, Roberta

Antonuzzo, Lorenzo

Anvar, Zahra

Apostolidis, Leonidas

Appetecchia, Marialuisa

Appolloni, Irene

Aquilanti, Elisa A.

Aragaki, Masato

Aragon-Ching, Jeanny B.

Arai, Ayako

Arai, Yasuaki

Aranda, Fernando

Arasanz Esteban, Hugo

Araujo, Arturo
Araújo-Gomes, Nuno

Arbajian, Elsa

Arbitrio, Mariamena

Arcovito, Alessandro

Ardizzoni, Andrea

Arechederra, María

Aregger, Michael

Arendowski, Adrian

Arendt, Lisa M.

Arezzo, Alberto

Argentiero, Antonella

Arguelles, Sandro

Arians, Nathalie

Aricò, Eleonora

Ariel, Amiram

Arif, Tasleem

Ariga, Katsuhiko

Arima, Yoshimi

Armant, Olivier

Armengol, Carolina

Armengot-Carbó, Miquel

Armocida, Daniele

Armstrong, Amy E.

Armstrong, Nicola

Arnaud, Jacquel

Arndt, Volker

Arnold, Dirk

Arosio, Daniela

Arribas, Joaquín

Arruga, Francesca

Arsenijevic, Tatjana

Artemaki, Pinelopi I.

Artico, Marco

Arul, Michael

Arús, Carles

Arvigo, Marica

Aryal, Uma K.

Asa, Sylvia L.

Asadpour, Vahid

Asghar, Muhammad Yasir

Asher, Nethanel

Ashihara, Eishi

Ashkarran, Aliakbar

Ashton, John

Ashton, Nicholas

Aspatwar, Ashok

Assaf, Chalid

Assié, Jean-Baptiste

Assy, Nimer

Asti, Mattia

Astigarraga, Itziar 
Astl, Jaromir

Atkins, Mardelle

Atrash, Shebli

Attarbaschi, Andishe

Atteritano, Marco

Attilio, Parisi

Attique, Muhammad

Attwa, Mohamed W.

$\mathrm{Au}$, Lewis

Auberger, Patrick

Audet-Walsh, Étienne

Audrius, Dulskas

Augusto, Danillo Gardenal

Aujayeb, Avinash

Auliac, Jean Bernard

Aureli, Anna

Aurer, Igor

Aurrand-Lions, Michel

Austin, Shane

Au-Yeung, George H.

Auzmendi-Iriarte, Jaione

Avanaki, Kamran

Avanzo, Michele

Avgeris, Margaritis

Avila, Matias

Avnet, Sofia

Avni, Dorit

Avni, Dror

Avolio, Alfonso W.

Avouac, Jerome

Awada, Gil

Ayuso, José María

Azevedo, Maria Manuel

Aziz, Khaled

Azizian, Azadeh

Azuma, Mizuki

Baba, Tsukasa

Babaian, Artem

Baburina, Yulia

Bacher, Ulrike

Bachet, Jean-Baptiste

Bacigalupo, Andrea

Bäcker, Daniel

Badal, Simone

Badescu, Minerva Codruta

Badiola, Iker

Baffy, Gyorgy

Bagby, Stefan

Bagnasco, Francesca

Bahcecioglu, Gokhan

Bahmad, Hisham
Bai, Li-Ping

Baig, Mohammad Hassan

Baik, Ku Youn

Bailey-Whyte, Maeve Louise

Bailleul, Justine

Baindara, Piyush

Baines, Joel D.

Baisi, Alessandro

Bajor, Malgorzata

Baker, Darren J.

Bakht, Martin

Balaban, Daniel Vasile

Balana, Carmen

Balasubramanian, Brinda

Balayssac, David

Balázs, Margit

Baldanzi, Gianluca

Baldari, Sergio

Balentova, Sona

Balestri, Francesco

Balgoma, David

Ballerini, Patrizia

Balogh, Andrea

Baltanás, Fernando C.

Bamdad, Cynthia

Bandapalli, Obul Reddy

Banday, Rouf

Bando, Etsuro

Banerjee, Debabrata

Baniahmad, Aria

Banik, Anna

Banito, Ana

Bano, Shazia

Bañuls-Roca, José

Bao, Ande

Bar, Eli E.

Barabutis, Nektarios

Baratta, Roberto

Barauskas, Giedrius

Barb, Adam

Barbagallo, Cristina

Barbara, Breznik

Barberio, Manuel

Barbu, Sorin T.

Barbui, Tiziano

Bardella, Chiara

Barderas, Rodrigo

Bar-Haim, Erez

Barisic, Karmela

Baritaki, Stavroula

Barker, Holly E. 
Barlier, Anne

Barnaba, Vincenzo

Barnas, Edyta

Barnoud, Thibaut

Barok, Márk

Barone, Biagio

Barr, Martin

Barragán, Isabel

Barreiro, Esther

Barrero, María José

Barresi, Valeria

Barresi, Vincenza

Barret, Maximilien

Barrott, Jared J.

Barsyte-Lovejoy, Dalia

Bartholomä, Mark Daniel

Bartl, Thomas

Bartlett, Edmund K.

Bartlett, Nancy L.

Bartoletti, Michele

Bartosinska, Joanna

Bartosz, Grzegorz

Bartůňková, Jiřina

Bartzsch, Stefan

Barui, Sugata

Barwe, Sonali P.

Barwick, Benjamin G.

Basadonna, Giacomo

Basak, Debasish

Basbous, Jihane

Baschant, Ulrike

Baselet, Bjorn

Basolo, Fulvio

Bassani-Sternberg, Michal

Basset-Séguin, Nicole

Bassi, Pierfrancesco Francesco

Basu, Reetobrata

Batalik, Ladislav

Bathula, Chandra Sekhar

Batie, Michael

Batina, Nikola

Batish, Mona

Battistelli, Cecilia

Bauchet, Luc

Bauckneht, Matteo

Baucum, Anthony J.

Bauer, Jessica

Bäuerle, Alexander

Baujat, Bertrand

Bauman, Glenn

Baumann, Tycho
Baumert, Brigitta G.

Bautista, Francisco

Baxevanis, Constantin N.

Baxter, Eva

Bayascas, Jose R.

Bayliss, Richard

Bazzichetto, Chiara

Beato, Miguel

Beauregard, Jean-Mathieu

Beaven, Anne W.

Bechmann, Nicole

Beck, Benjamin

Beck, Marcus

Beck, William T.

Becker, Aline P.

Becker, Jürgen

Bednarsch, Jan

Bedogni, Barbara

Beebe, Stephen

Beenakker, Jan-Willem

Beer, David G.

Beer, Meinrad

Begnaud, Abbie L.

Behling, Felix

Behrens, Kira

Beig, Niha

Beinat, Corinne

Bekiaris, Vasileios

Bekkers, Ruud L. M.

Bélanger, Kasandra

Belfiore, Antonino

Belickova, Monika

Belka, Claus

Bellamri, Madjda

Belli, Maria Luisa

Belli, Mauro

Bellini, Maria Irene

Bello, Ibrahim Olajide

Belmonte-Beitia, Juan

Belting, Mattias

Benajiba, Lina

Ben-Chetrit, Nir

Bendas, Gerd

Ben-David, Yaacov

Benelli, Roberto

Benesch, Matthew

Benevene, Paula

Benistant, Christine

Bennani-Baïti, Barbara

Bennardo, Luigi

Bennouna, Jaafar 
Bensadoun, René-Jean

Ben-Salem, Salma

Bentel, Jacqueline M.

Bentivegna, Angela

Benzaquen, Jonathan

Bera, Amit

Berardi, Anna Concetta

Berasain, Carmen

Berdel, Wolfgang E.

Berdyshev, Evgeny V.

Berentsen, Sigbjørn

Beretta, Giovanni L.

Berezovski, Maxim V.

Bergamini, Carlo

Bergamini, Christian

Bergandi, Loredana

Bergantini, Laura

Berger, Adam C.

Berger, Claire

Berger, Massimo

Berkowitz, Michaela

Bernardi, Simona

Bernardini, Giovanni

Bernhardt, Peter

Bernstein, Lori J.

Berrino, Liberato

Berselli, Mattia

Berthold, Frank

Berthon, Annabel S.

Bertolini, Francesco

Bertoni, Francesco

Bertozzi, Serena

Berveiller, Paul

Berzins, Stuart

Besic, Nikola

Besse, Lenka

Besson, Caroline

Betancourt, Lazaro

Betés, Maite Teresa

Bette, Michael

Betz-Stablein, Brigid

Beuriat, Pierre-Aurélien

Bevilacqua, Alessandro

Bewersdorf, Jan Philipp

Beylot-Barry, Marie

Beyreuther, Elke

Bezu, Lucillia

Bhagirath, Divya

Bharati, Sanjay

Bhardwaj, Rajesh

Bhardwaj, Vikas
Bhargava, Rhea

Bhat, Ambarish P.

Bhat, Kamakoti

Bhat, Raksha

Bhattacharya, Abhisek

Bhattacharya, Rajat

Bhattacharya, Santanu

Bhattacharya, Somanon

Bhattacharyya, Sohinee

Bhawal, Ujjal Kumar

Bhowmick, Neil A.

Biamonte, Flavia

Bianchi, Alberto

Bianchi, Lorenzo

Bianchi, Marzia

Bianchi, Serena

Bianchini, Laurence

Bianchini, Rodolfo

Bianco, Maria Rita

Bianco, Roberto

Bianconi, Fortunato

Biasoli, Irene

Biassoni, Roberto

Bibault, Jean-Emmanuel

Bibikova, Olga

Bidoli, Ettore

Biedermann, David

Bielawska-Pohl, Aleksandra

Bielawski, Kornel

Bielinsky, Anja Katrin

Bieńkowski, Michał

Biersack, Bernhard

Biewald, Eva

Biffo, Stefano

Biffoni, Marco

Bigley, Austin B.

Bijlsma, Maarten F.

Bikas, Athanasios

Bilbao, Daniel

Bilger, Andrea

Bill, Marius

Bilodeau, Marc

Bilski, Jan

Binotto, Gianni

Biondi, Antonio Giuseppe

Birkeland, Andrew C.

Birkó, Zsuzsanna

Bishayee, Anupam

Bishehsari, Faraz

Bishnupuri, Kumar S.

Bismar, Tarek 
Bistulfi, Gaia

Bitzer, Michael

Björkblom, Benny

Blache, Philippe

Black, Joshua C.

Blackburn, Jessica

Blandino, Giovanni

Blank, Alan T.

Blank, Susanne

Blank, Volker

Blanquart, Christophe

Blattner, Christine

Blay, Jaen-Yves

Bleckmann, Annalen

Bleeker, Maaike C. G.

Blenda, Anna

Bloch, Wilhelm

Boada, Aram

Boakye, Daniel

Boaventura, Paula

Bobba, Kondapa Naidu

Bobiński, Marcin

Boccalatte, Francesco

Bochtler, Tilmann

Boehm, Katja

Bogacka, Iwona

Bogani, Giorgio

Boggio, Elena

Bogucki, Jacek

Bohmer, Dirk

Boileau, Etienne

Boilève, Alice

Boinska, Joanna

Boix, Loreto

Boj, Sylvia F.

Bojarska-Junak, Agnieszka A.

Bojunga, Joerg

Bolam, Kate A.

Boldrini, Luca

Bolm, Louisa

Bolomsky, Arnold

Bommareddy, Praveen

Bommireddy, Ramireddy

Boms, Stefanie

Bomsztyk, Karol

Bonavita, Eduardo

Bonetti, Andrea

Bonferoni, Maria Cristina

Bongiovanni, Alberto

Bongiovanni, Massimo

Bonifacio, Massimiliano
Bonin, Serena

Bonino, Ferruccio

Bonnet, Pierre-Antoine

Bonnici, Vincenzo

Bono, Hidemasa

Bonora, Elena

Boo, Yong Chool

Boon, Siaw

Boppana, Nithin B.

Borcherding, Dana C.

Borcherding, Nicholas

Borg, David

Borgia, Jeffrey A.

Borgna, Francesca

Borisenkov, Mikhail F.

Borkowetz, Angelika

Borkowska, Edyta Marta

Bornfeld, Norbert

Bornhaeuser, Martin

Borrelli, Paola

Børset, Magne

Borson-Chazot, Françoise

Borstnar, Simona

Bortner, Carl D.

Borzi, Cristina

Borzio, Mauro

Boschert, Verena

Bose, Debojit

Bosi, Alberto

Bosnjak, Masa

Bost, Frédéric

Bostan, Marinela

Boström, Jan Patrick

Bôta, Attila

Botella, Luisa María

Botta, Cirino

Bottalico, Lucrezia

Botticelli, Andrea

Botton, Thomas

Bouchal, Pavel

Boucheix, Claude

Boudreau, Howard E.

Bougen-Zhukov, Nicola

Bouleuc, Carole

Bouley, Renee

Bouley, Richard

Boulkroun, Sheerazed

Boulter, Luke

Bourdon, Emmanuel

Bourdon, Jean-Christophe

Bousbaa, Hassan 
Bousquet, Corinne

Bousquet, Guilhem

Boussios, Stergios

Bouvier, Anne-Marie

Bouwman, Peter

Bove, Aldo

Bovell, Liselle

Bowen, Deborah

Bowman, Caitlyn

Boyer, Thomas

Boyle, Glen M.

Bozec, Alexandre

Bożena, Leszczyńska-Gorzelak

Bozic, Ivana

Bozic, Josko

Braathen, Lasse

Brabek, Jan

Bracht, Jillian Wilhelmina Paulina

Brady, Adrian

Brägelmann, Johannes

Braicu, Cornelia

Brakenhielm, Ebba

Brambilla, Dario

Branca, Jacopo Junio Valerio

Brancaccio, Mariarita

Brancati, Nadia

Brandal, Petter

Brandi, Nicolò

Brandt, Burkhard

Branzei, Dana

Brasher, Penelope M. A.

Brassart-Pasco, Sylvie

Bratashov, Daniil N.

Brattsand, Maria

Braun, Felix

Braun, Thorsten

Brauner, Edoardo

Braunstein, Evan M.

Brechbuhl, Heather

Brégier, Frédérique

Breier, Georg

Bremer, Edwin

Brenet, Fabienne

Brennan, Donal

Brennan, Greg

Brenner, J. Chad

Brenner, John Chad

Bresciani, Roberto

Bresson-Bepoldin, Laurence

Brézillon, Stéphane

Bria, Emilio
Brichkina, Anna

Brieger, Angela

Brindley, David

Brioli, Annamaria

Brito, Catarina

Broccoli, Alessandro

Brockmeyer, Phillipp

Brodska, Barbora

Brody, Jonathan R.

Broekgaarden, Mans

Broggi, Giuseppe

Broggini, Massimo

Bronte, Fabrizio

Brooke, Greg

Brooks, Allen

Brosens, Erwin

Brossart, Peter

Brosseau, Jean-Philippe

Brouchet, Anne

Brounais-Le-Royer, Bénédicte

Brown, James

Brown, Jeffrey $W$.

Brown, Justin

Brown, Nelson

Brown, Ronald B.

Brozovic, Anamaria

Brozzetti, Stefania

Brugnoli, Federica

Brummer, Tilman

Bruni, Laia

Brunner, Markus

Brunner, Maximilian

Brunner, Thomas

Brünnert, Daniela

Bruno, Antonino

Bruno, Federico

Bruzzaniti, Placido

Bruzzese, Francesca

Bryant, Helen

Buccisano, Francesco

Buchler, Tomas

Buck, Michael D.

Buckle, Ashley

Buechert, Martin

Buffi, Nicolòmaria

Bugada, Dario

Bugalho, Maria João

Buijs, Jeroen T.

Bujanda, Luis

Bujko, Mateusz

Bukkuri, Anuraag 
Bulat, Tanja

Bulfone, Giampiera

Bulin, Anne-Laure

Bunce, Chris M.

Bundschuh, Ralph A.

Bunevičius, Adomas

Bunin, Greta R.

Buoncervello, Maria

Burchell, Joy

Burckhardt, Christoph

Burdach, Stefan

Burdak-Rothkamm, Susanne

Burden, Roberta

Bure, Irina V.

Burek, Malgorzata

Burger, Michael C.

Burgett, Anthony W. G.

Bürglin, Thomas R.

Burgos-Aceves, Mario Alberto

Burkard, Mark E.

Burnell, Matthew

Burra, Patrizia

Burster, Timo

Buscail, Louis

Busch, Jonas

Busch, Maike

Busetto, Gian Maria

Buske, Christian

Busse, Antonia

Büsselberg, Dietrich

Bussolati, Ovidio

Bustoros, Mark

Buszewska-Forajta, Magdalena

Butler, Eboneé

Büttner, Reinhard

Butturini, Elena

Buzdin, Anton

Buzza, Mark

Bychkov, Andrey

Bye, Alexander P.

Byrne, Hugh

Byung Chang, Kim

Caballero-Villarraso, Javier

Cabañas, Carlos

Cackowski, Frank

Cadamuro, Massimiliano

Cadilha, Bruno L.

Cadossi, Ruggero

Cadoux-Hudson, Thomas A. D.

Cadum, Ennio

Caers, Jo
Caetano, Joana

Caffo, Maria

Cafri, Gal

Cagini, Lucio

Cagnazzo, Celeste

Cagnin, Stefano

Cai, Chunyu

Cai, Lisheng

Caimi, Paolo

Cairrão, Elisa

Calcabrini, Annarica

Caligo, Maria Adelaide

Calò, Valentina

Calogero, Antonella

Caltabiano, Rosario

Calvaruso, Marco

Calvisi, Diego Francesco

Calvo, Gabriel F.

Camacho-Arroyo, Ignacio

Cambien, Béatrice

Cameron, Donald

Cameselle-Teijeiro, Jose Manuel

Cammarata, Francesco

Campa, Carlo Cosimo

Campagna, Roberto

Campagne, Olivia

Campagnola, Paul J.

Campana, Davide

Campanella, Fabio

Campbell, Kerry

Campione, Severo

Campolo, Federica

Campolo, Michela

Campos, Camila D. Madeira

Camps, Jordi

Canbay, Ali

Canbay, Emel

Canberk, Sule

Candoni, Anna

Canela, Enric I.

Canesin, Giacomo

Canevari, Silvana

Cannarozzo, Giovanni

Cantin, André M.

Cantini, Giulia

Canu, Letizia

Cañueto, Javier

Canzona, Mollie

Cao, Kim

Cao, Yihai

Capaccio, Pasquale 
Capece, Daria

Capeloa, Tania

Capiod, Thierry

Capobianco, Giampiero

Capoluongo, Ettore

Caponio, Vito Carlo Alberto

Cappariello, Alfredo

Cappelletti, Vera

Cappello, Paola

Capuano, Alessandra

Capula, Mjriam

Carai, Andrea

Caramalho, Iris

Caramujo, Maria José

Caratozzolo, Mariano Francesco

Carbognin, Luisa

Carbonaro, Luca A.

Carbone, Antonino

Carbone, Daniela

Carcereri De Prati, Alessandra

Carchman, Evie $\mathrm{H}$.

Cardile, Venera

Cardillo, Giuseppe

Cardobi, Nicolò

Carè, Alessandra

Care, Andrew

Carey, Renee

Carlberg, Carsten

Carleo, Alfonso

Carlisi, Daniela

Carloni, Riccardo

Carlos, Ana Rita

Carmen, Mejía

Carneiro, Patrícia

Carnesecchi, Julie

Caro, Carlos

Carotenuto, Pietro

Carpizo, Darren R.

Carr, Robert A.

Carrà, Giovanna

Carrano, Anna

Carraro, Fabio

Carrer, Alessandro

Carrera, Pilar

Carreras, Joaquim

Carrier, France

Carriero, Alessandro

Carruthers, Ross

Carter, Wayne

Caruana, Ignazio

Carullo, Gabriele
Caruntu, Ana

Caruntu, Constantin

Carus, Andreas

Caruso, Gerardo

Carvalho, Edmund

Carvalho, Joana

Casadio, Paolo

Casado, Pedro

Casalou, Cristina

Casanova Acebes, María

Casanova, Emilio

Casares, Noelia

Casas, Josefina

Cascini, Giuseppe Lucio

Cascio, Sandra

Casey, Jennifer

Casimiro, Sandra

Cassano, Enrico

Cassim, Shamir

Castelli, Vanessa

Castelo-Branco, Pedro

Castillo-Tong, Dan Cacsire

Castro, Flávia

Castro-Vega, Luis Jaime

Catchpoole, Daniel

Catela Ivkovic, Tina

Catic, Andre

Catino, Annamaria

Cattrini, Carlo

Cauli, Omar

Caux, Christophe

Cava, Claudia

Cavaco, Daniela

Cavalieri, Stefano

Cavallari, Eleonora

Cavalloni, Giuliana

Cavazzoni, Andrea

Cavic, Milena

Cavo, Alessia

Cayrefourcq, Laure

Cazacu, Irina

Caziuc, Alexandra

Cazzola, Emiliano

Cea, Michele

Cecinati, Valerio

Celentano, Egidio

Cenariu, Mihai

Cenni, Vittoria

Cepeda, Santiago

Ceppi, Lorenzo

Ceraline, Jocelyn 
Cerchia, Laura

Cerchione, Claudio

Cerdán, María-Esperanza

Cernuda, Rafael

Cervera, José

Cervigón, Raquel

Cerwenka, Herwig

Cescon, Matteo

Cesetti, Tiziana

Chabay, Paola

Chaber, Radosław

Chabot, Benoit

Chacko, Jenu Varghese

Chahwan, Richard

Chakrabarti, Jayati

Chakraborty, Abhishek A.

Chakraborty, Anirban

Chakraborty, Sayan

Chakroborty, Debanjan

Challis, Benjamin G.

Chan, Jason

Chan, Jason Y. K.

Chan, Karen Kar Loen

Chan, Keefe T.

Chan, Sheng-Chieh

Chan, Waikin

Chandra, Janin

Chandrasekaran, Akshaya

Chang, Ching-Jin

Chang, Eunju

Chang, Hsueh-Wei

Chang, Jer-Hao

Chang, Julia Yu-Fong

Chang, Meichi

Chang, Nan-Shan

Chang, Shu-Chun

Chang, Wei-Lun

Chang, Wei-Min

Chang, Wen Tsan

Chang, Wen-Wei

Chang, Yu Chan

Chapman, Paul B.

Chardes, Thierry

Charli-Joseph, Yann Vincent

Charriere, Guillaume

Chatain, Nicolas

Chatelut, Étienne

Chatterjee, Aniruddha

Chatterjee, Nimrat

Chatterjee, Sampurna

Chattopadhyay, Sajal K.
Chauvie, Stephane

Chaves, Susana Rodrigues

Cheema, Umber

Cheifetz, Rona

Chelakkot, Chaithanya

Chelikani, Prashen

Chellaiah, Meena

Chemello, Liliana

Chen, Bang-Bin

Chen, Chang-Han

Chen, Chaoling

Chen, Chien-Chin

Chen, Chih-Shan Jason

Chen, Chi-Long

Chen, Chung-Hsin

Chen, Chun-Han

Chen, Chun-Liang

Chen, Dexi

Chen, Georgia Zhuo

Chen, Guan

Chen, Hanying

Chen, Hao

Chen, Hongwei

Chen, Hongwu

Chen, $\mathrm{Hu}$

Chen, Huarong

Chen, Jinbiao

Chen, Jing

Chen, Jui-Chieh

Chen, Ke-Cheng

Chen, Kuang-Chi

Chen, Liching

Chen, Lihua

Chen, Longwen

Chen, Mei-Chuan

Chen, Ming

Chen, Mingsheng

Chen, Mingyi

Chen, Pai-Sheng

Chen, Rong-Jane

Chen, San-Yuan

Chen, Sean Chun Chang

Chen, Shaw-Ji

Chen, Sheng-Hung

Chen, Shihlung

Chen, Shin-Cheh

Chen, Shukun

Chen, Si-Tong

Chen, Weiqiang

Chen, Yann-Jang

Chen, Yifan 
Chen, Yih Yuan

Chen, Yih-Fung

Chen, Yuchih

Chen, Yu-Chih

Chen, Zeyu

Chen, Zhe-sheng

Chen, Zigui

Cheng, Alfred Sze-Lok

Cheng, Chia-Hsiung

Cheng, Chunchia

Cheng, Chunming

Cheng, Henrich

Cheng, Hsutang

Cheng, Hung-Chi

Cheng, Jing-Jy

Cheng, Kun

Cheng, Ming-Huei

Cheng, Tsu-Yao

Cheong, Jit Kong

Chepelev, Leonid

Cherdyntseva, Nadezhda V.

Cherkasova, Olga

Chernyshev, Vasiliy

Cherukula, Kondareddy

Chessa, Luciana

Cheung, Kwok-Leung

Cheung, Martin

Chevalier, François

Chhabra, Gagan

Chi, Hsiang-Cheng

Chi, Kwan Hwa

Chia, Jean-San

Chiang, J. M.

Chiang, Naijung

Chiang, Weichung

Chiang, Ying-Cheng

Chiang, Yi-Ting

Chiara, María-Dolores

Chiarion-Sileni, Vanna

Chiavassa, Sophie

Chiba, Hideyuki

Chicano-Gálvez, Eduardo

Chiche, Johanna

Chichiarelli, Silvia

Chiefari, Eusebio

Chien, Chih-Yen

Chien, Chun-Ru

Chihara, Dai

Chimenz, Roberto

Chinen, Ludmilla

Chintala, Sreenivasulu
Chiocchetti, Annalisa

Chiofalo, Maria Grazia

Chirgwin, John M.

Chitale, Shalaka

Chitcholtan, Kenny

Chithrani, Devika

Chittiboina, Prashant

Chiu, Ching-Feng

Chiu, Hua-Sheng

Chiu, Ingming

Chiu, Norman H. L.

Chiuri, Vincenzo Emanuele

Cho, Anna

Cho, Choifong

Cho, Jae Hee

Cho, Jeonghee

Cho, May

Cho, Shih-Feng

Cho, Young-Youn

Chodick, Gabriel

Choi, Changhoon

Choi, Hyo Geun

Choi, Jiyeon

Choi, Min-chul

Choi, Siu Wai

Choi, Young

Chong, Geoff

Chong, Irene Yu-Shing

Chong, Yosep

Chou, Angela S.

Chou, Cheng-Yang

Chou, Chih-Hung

Chou, Chih-Ming

Chou, Wen-Chien

Choudhury, Parichoy Pal

Chrétien, Anne-Sophie

Christen, Thomas

Christian, Selinger

Christiansen, Hans

Christie, Michael

Christmann, Markus

Christopher, LaRocca

Christopoulos, Petros

Chrzanowska, Alicja

$\mathrm{Chu}$, Cheng-Ying

$\mathrm{Chu}$, Ching-Liang

$\mathrm{Chu}$, Wai Kit

$\mathrm{Chu}$, Xiangping

Chuang, Shih-Sung

Chuang, Show-Mei

Chuang, Wan-Long 
Chulián, Salvador

Chun, Sejong

Chun, Yang-Sook

Chung, Byung Min

Chung, Chi-Li

Chung, Ching-Hu

Chung, Christine

Chung, Felicia Fei-Lei

Chung, Jae Hoon

Chung, Joon-Yong

Chuntao, Zhao

Chuntova, Pavlina D.

Churg, Andrew

Churov, Alexey

Chutipongtanate, Somchai

Ciardo, Silvana

Ciarimboli, Giuliano

Ciccarelli, Renata

Cicchi, Stefano

Ciccolini, Joseph

Cicènas, Saulius

Cicero, Arrigo

Cichorek, Miroslawa

Cidre-Aranaz, Florencia

Čierniková, Soňa

Cieslar, Grzegorz

Çiftçi, Halil İbrahim

Ciminale, Vincenzo

Cimini, Annamaria

Cimmino, Amelia

Cimmino, Flora

Cingolani, Antonella

Cinque, Alessandra

Cinque, Benedetta

Cioce, Mario

Cione, Erika

Cipak Gasparovic, Ana

Cipak, Lubos

Cipolletti, Manuela

Ciribilli, Yari

Ciriolo, Maria

Ciszewski, Wojciech Michał

Citro, Simona

Cittadini, Giuseppe

Citterio, Davide

Cives, Mauro

Civita, Prospero

Clark, Dana Farengo

Clark, Geoffrey J.

Clark, John R.

Clemons, Nicholas
Clifford, Rachael E.

Clynes, David

Cocco, Stefania

Cochonneau, Denis

Cochrane, Dawn R.

Cocozza, Sergio

Cocuzza, Salvatore

Cocuzza, Salvatore Giuseppe

Codacci-Pisanelli, Giovanni

Coffey, Kelly

Cogliati, Bruno

Cognasse, Fabrice

Cohen, Idan

Cohen, Marie

Cohen-Gogo, Sarah

Cokan, Kaja Blagotinšek

Colaço, Bruno Jorge

Colavito, Sierra A.

Cole, Laura

Cole, Trevor R. P.

Colecchia, Maurizio

Colell, Anna

Coletti, Dario

Collavin, Licio

Collawn, Sherry S.

Collins, Christopher M.

Collins, Denis

Collura, Ada

Colnot, Sabine

Colombo, Michela

Colomo, Lluís

Colonna, Giovanni

Colquhoun, Steven D.

Conceição, Natércia

Conciatori, Fabiana

Conde-Estévez, David

Condelli, Valentina

Condello, Salvatore

Condorelli, Daniele Filippo

Condorelli, Gerolama

Conigliaro, Alice

Conlon, Neil

Connolly, Michael

Conoci, Sabrina

Conrad, David M.

Consalvi, Valerio

Console, Lara

Consonni, Francesca Maria

Constantinescu, Ileana

Constantinidou, Anastasia

Constanzo, Julie 
Contaldo, Maria

Conti, Alfredo

Conti, Pio

Cools-Lartigue, Jonathan

Copeland, Nikki

Copland, Mhairi

Coppa, Jorgelina

Coppes, Rob

Coppey, Mathieu

Corbet, Cyril

Corbo, Vincenzo

Cordani, Nicoletta

Cordenonsi, Michelangelo

Corey, Seth

Cormio, Gennaro

Cornelis, Francois

Cornelius, Denise

Corniola, Marco V.

Corominas, Montserrat

Correnti, Margherita

Corsaro, Alessandro

Corsi, Fabio

Corsi, Lorenzo

Corso, Simona

Corti, Chiara

Corvalán, Alejandro H.

Corvatta, Laura

Corvigno, Sara

Costa, Clotilde

Costa, José Luis

Costa, Maria Do Céu

Costa, Marta

Costarelli, Leopoldo

Costes, Nicolas

Cote, Gregory M.

Côté, Hélène

Cotoi, Laura

Cottini, Francesca

Couchman, John

Coucke, Wim

Couttet, Philippe

Couvineau, Alain

Covarelli, Piero

Coveñas, Rafael

Coward, Jermaine

Cowden Dahl, Karen

Cowley, Shaun M.

Cox, Dianne

Coyle, Beth

Crabb, John

Craig, Amanda J.
Craig, Morgan

Craig, Stephanie G.

Cramer, Gwendolyn M.

Cravo, Marília

Crawford, Howard C.

Crawford, Lisa

Crescioli, Silvia

Creta, Massimiliano

Crezee, Hans

Crinò, Stefano Francesco

Crisa, Elena

Crisafulli, Giovanni

Cristalli, Giovanni

Cristina, Müller

Cristofanilli, Massimo

Cronauer, Marcus

Cronin, Patricia A.

Crooks, Daniel R.

Cros, Jérôme

Crosas, Bernat

Croset, Martine

Crowe, David

Crucitta, Stefania

Crucitti, Pierfilippo

Crulhas, Bruno Pereira

Crum, Christopher

Csapo, Edit

Csizmadi, Ilona

Csoka, Monika

Cuadrado, Myriam

Cuatrecasas, Miriam

Cubero, Francisco Javier

Cuccia, Francesco

Cuenda, Ana

Cui, Feng

Cullen, Jennifer

Cumbo, Cosimo

Cumming, Paul

Cuneo, Antonio

Cunningham, Jessica J.

Cuocolo, Renato

Currie, Margaret

Cuschieri, Kate

Cusumano, Davide

Cvek, Jakub

Cvek, Urska

Cymbaluk-Ploska, Aneta

Czarnecka, Karolina

Czarniecka, Agnieszka

Czarnomysy, Robert

Czyż, Jarosław 
D'Agnano, Igea

D'agostino, Donna

D'Agostino, Mattia

D'Agostino, Vito Giuseppe

D'Aguanno, Simona

D'alessio, Alessio

D'Alò, Francesco

D'Angelo, Francesco

D'arcy, Pádraig

D'Errico, Antonietta

D'Onofrio, Giuseppe

D'Onofrio, Mirko

D'Onofrio, Nunzia

D'Orazi, Valerio

Da Silva, Luís Pinto

Da Vià, Matteo

Dabaja, Bouthaina

Dada, Reyad

Dahal, Lekh

Dai, Wei

Dal Bo, Michele

Dal Col, Jessica

Dall, Genevieve

Dalla Palma, Anna Benedetta

Dalla Vecchia, Laura Adelaide

Damaghi, Mehdi

Damascelli, Bruno

Damato, Bertil

Dambrauskas, Zilvinas

Damiani, Daniela

Damm, Frederik

Dammann, Philipp

Dammann, Reinhard

Daneshvar, Michael A.

Danforth, David N.

Dang, Tuyen T.

Daniels, Mark J.

Danila, Edvardas

Danilenko, Michael

Danis, Judit

Dann, Eldad

Danti, Serena

Danysh, Heather E.

Daponte, Alexandros

Darbin, Olivier

Dario, Mandato Vincenzo

Dariš, Barbara

Darling-Reed, Selina F.

Darwiche, Kaid

Das, Gokul

Das, Nupur
Das, Prasanta

Dasari, Pallave

Dasari, Subramanyam

Dasgupta, Paramita

Dash, Sabyasachi

Dash, Srikanta

Daskalakis, Kosmas

Dastoli, Stefano

Daubon, Thomas

David Cordonnier, Marie Hélène

David, Mutch

Davidson, Brian R.

Davie, Judith

Dawnay, Anne

Dawson, Michelle R.

Day, Andrew S.

Day, Chi-ping

Day, Regina M.

Dayyani, Farshid

De Araújo Júnior, Raimundo Fernandes

De Assis, Leonardo V. M.

De Baene, Wouter

De Bari, Lidia

De Barrios, Oriol

De Bree, Eelco

De Bree, Remco

De Carvalho, Litia Alves

De Clercq, Eva

De Cristofaro, Tiziana

De Felice, Francesca

De Gregorio, Vincenza

De Haas, Valerie

De Jaeghere, Emiel A.

De Jong, Daphne D.

De Jong, Florus C.

De Jong, Igle Jan

De Jong, Monique

De Juan Romero, Camino

De Klein, Annelies

De La Parra, Columba

De La Parte, Borja Herrero

De La Peña, Francisco Ayala

De La Roche, Marc

De La Rosa, Jorge

De La Rubia, Javier

De Ligt, Kelly

De Los Toyos, Juan R.

De Luca, Anastasia

De Marchi, Elena

De Marco, Federico

De Maria, Raffaella 
De Martino, Maria Cristina

De Matos Simoes, Ricardo

De Mattia, Elena

De Melo Porcari, Andréia

De Melo-Diogo, Duarte

De Mestier, Louis

De Meyts, Pierre

De Miglio, Maria Rosaria

De Miguel-Pérez, Diego

De Nigris, Filomena

De Nonneville, Alexandre

De Nully Brown, Peter

De Paoli, Antonino

De Petro, Giuseppina

De Pinho, Paula Guedes

De Reijke, Theo M.

De Reuver, Philip

De Ridder, Mischa

De Robertis, Mariangela

De Rossi, Anita

De Santis, Maria Chiara

De Santo, Carmela

De Sarkar, Navonil

De Sepulveda, Paulo

De Sousa-Coelho, Ana Luísa

De Terlizzi, Francesca

De Totero, Daniela

De Velasco, Guillermo

De Velasco, Marco A.

De Vicente, Juan Carlos

De Vincenzo, Rosa

De Vita, Alessandro

De Wilde, Bram

De, Tanima

Deaglio, Silvia

Dealwis, Chris G.

Dean, Michael

Deandreis, Désirée

Deantonio, Letizia

Deb, Subrata

Decarolis, Boris

Deckert, Marcel

De-Colle, Chiara

Decottignies, Anabelle

Deevska, Gergana

Defilippi, Paola

Dekanic, Andrea

Del Bianco, Paola

Del Favero, Giorgia

Del Giudice, Francesco

Del Mastro, L.
Del Mistro, Annarosa

Del Rivero, Jaydira

Del Rosso, Mario

Del Sal, Giannino

Del Vecchio, Silvana

Del Zingaro, Michele

Del Zotto, Genny

Delhomme, Tiffany M.

Delie, Florence

Dell'Amore, Andrea

Dell'Aversana Orabona, Giovanni

Della Bella, Chiara

Della Pepa, Giuseppe Maria

Della Valle, Serena

Delle Cave, Donatella

Dellis, Olivier

Dello Sbarba, Persio

Demehri, Shawn

Demetter, Pieter

Demicheli, Romano

De-Miguel, Manuel

Demirci, Hakan

Demirkhanyan, Lusine $\mathrm{H}$.

Deng, Qu

Deng, Youping

Dengel, Andreas

Denis, Jerome

Denisov, Evgeny

Denizot, Yves

Dennany, Lynn

Dennis, Leslie K.

Denys, Alban

Deore, Prashant S.

Depau, Lorenzo

Depciuch, Joanna

Deptuła, Milena

Derks, J. L.

Derksen, Patrick W. B.

Derlin, Thorsten

Derwich, Katarzyna

Desantis, Vanessa

Desaphy, Jean-François

Desouki, Mohamed M.

Deutsch, Alexander

Devaux, Bertrand

Devillier, Raynier

Devine, Katie

Dey, Debasish Kumar

Dey, Kamol

Dey, Nandini

Dey, Priyanka 
Dey, Shuvashis

Dhimolea, Eugen

Dhungel, Bijay

Di Agostino, Silvia

Di Cataldo, Andrea

Di Donato, Marzia

Di Franco, Simone

Di Giammartino, Dafne Campigli

Di Grezia, Graziella

Di Mario, Francesco

Di Nunno, Vincenzo

Di Palma, Tina

Di Paola, Valerio

Di Pietro, Pietro

Di Renzo, Livia

Di Renzo, Maria Flavia

Di Rorà, Andrea Ghelli

Di Saverio, Salomone

Di Sipio, Tracey

Di Spiezio Sardo, Attilio

Di Tommaso, Luca

Di Trapani, Ettore

Diacono, Domenico

Dianzani, Umberto

Diaz, Abbey

Diaz, Fernando

Diaz-Flores, Lucio

Díaz-García, Diana

Díaz-Rodríguez, Elena

Didier, Christine

Didilescu, Andreea

Diefenbach, Russell J.

Diepstra, Arjan

Dierge, Emeline

Dieterich, Lothar

Dietrich, Charles

Dijk, Frederike

Dillman, Robert O.

Dimanche-Boitrel, Marie-Thérèse

Dimitrakopoulos, Foteinos-Ioannis

Dimitriadis, Emilios K.

Dimri, Manali

Dinalankara, Wikum

Dindelegan, George Calin

Ding, Han-Fei

Ding, Keyue

Ding, Lingwen

Dinh, Claire T.

Dini, Valentina

Dinic, Jelena

Dinis-Oliveira, Ricardo Jorge
Dionyssiou, Dimitrios

Diorio, Caroline

Dirven, Linda

Disciglio, Vittoria

Dittmer, Jurgen

Dixon, Stephanie B.

Djerada, Zoubir

Dmitriev, Ruslan

Dmitrieva, Renata I.

Doberstein, Kai

Dobos, Gabor

Dobrescu, Amadeus

Dobruch, Jakub

Dobruch-Sobczak, Katarzyna

Dobrzyn, Pawel

Doghman, Mabrouka

Doglio, Alain

Dogra, Samrita

Doherty, Mark

Dolivo, David

Domagala-Kulawik, Joanna

Domanski, Henryk

Domenech, Maribella

Domenico, Mascagni

Domingo, Enric

Domínguez-Valentín, Mev

Dommering, Charlotte J.

Domoto, Takahiro

Donahoe, Laura

Donati, Chiara

Dong, Liang

Dong, Lianhua

Dongryull, Oh

Donner, Davide

Doretto, Paolo

Doria, Cataldo

Dorleijn, Desirée M. J.

Dorling, Leila

Dormond, Olivier

Dorsman, Josephine

Dos Anjos Pires, Maria

Dotan, Zohar

Draghi, Arianna

Dragoni, Silvia

Drake, Penelope M.

Drakos, Elias

Dreassi, Elena

Dreyer, Tobias F.

Drid, Patrik

Driessen, Christoph

Dröge, Leif Hendrik 
Drott, Jenny

Droz, Jeanpierre

Drozd-Sokołowska, Joanna

Dryahina, Kseniya

Du, Juan

$\mathrm{Du}$, Wenting

Du, Xuexiang

Duan, Jialei

Duarte, Antonio

Dubash, Taronish Dorab

Dubois, Claire M.

Dubois, Ludwig

Dubrovska, Anna

Duc, Nguyen Minh

Duca, Laurent

Duca, Lorena

Duchi, Serena

Ducret, Thomas

Duensing, Stefan

Dufresne, Armelle

Duggan, Shane

Dugué, Pierre-Antoine

Duhamel, Marie

Dulskas, Audrius

Dumaz, Nicolas

Dumic, Jerka

Dumitrascu, Traian

Dummer, Reinhard

Dumont, Frédéric

Dumont, Sarah Noémie

Dumoulin, Franz Ludwig

Dunlap, Neal

Dunlop, Elaine

Duque-Afonso, Jesus

Durán-Poveda, Manuel

Duranti, Claudia

Duranti, Simona

Durdik, Matus

Durgo, Ksenija

Durlik, Magdalena

Durocher, Francine

Durrieu, Francoise

Duskey, Jason Thomas

Duso, Bruno

Duś-Szachniewicz, Kamila

Dutil, Julie

Dutta, Arijit

Dutta, Pranabananda

Dutta, Samikshan

Düzgüneş, Nejat

Dvorankova, Barbora
Dwarkasing, Roy S.

Dye, Danielle E.

Dymock, Brian William

Dymova, Maya

Dziegiel, Piotr

Dziegielewska, Barbara

Dzięgielewska-Gęsiak, Sylwia

Dzikiewicz-Krawczyk, Agnieszka

East, Michael P.

Eastman, Alison

Eatrides, Jennifer M.

Eaves-Pyles, Tonyia

Eberhart, Charles

Eble, Johannes

Ebstein, Frédéric

Eccher, Albino

Echchannaoui, Hakim

Echevarría, Enrique

Eckert, Alexander

Eckert, Alexander W.

Eckrich, Jonas

Eckschlager, Tomas

Edderkaoui, Mouad

Edeline, Julien

Eden, Amir

Edfors, Fredrik

Edwards, Claire

Eeltink, Corien M.

Eersels, Jos L.

Efebera, Yvonne A.

Eferl, Robert

Efremov, Dimitar

Egashira, Nobuaki

Egger, Jan

Ehata, Shogo

Ehrlich, Melanie

Eichbaum, Michael

Eichenauer, Dennis A.

Eichhorn, Florian

Eichhorn, Pieter

Eichler, Martin

Eisendle, Klaus

Eisenstat, David D.

Eisermann, Kurtis

Eiz-Vesper, Britta

Ekert, Paul G.

Ekkernkamp, Axel

Eksi, Sebnem Ece

Eksioglu, Erika A.

El Sharouni, Mary-Ann

Elander, Nils 
Eldfors, Samuli

Elfer, Katherine

El-Gewely, Mohamed Raafat

Eliçin, Olgun

Eliseeva, Irina

Elkabets, Moshe

Elkrief, Arielle

Ellinger, Jörg

El-Mesery, Mohamed

Eloranta, Sandra

El-Rifai, Wael

Elsawa, Sherine F.

Elsayad, Khaled

Elsherbini, Ahmed M.

Elsherbiny, Marwa

Emfietzoglou, Dimitris

Emons, Günter

Emran, Abdullah Al

Emri, Gabriella

Endo, Makoto

Engels, Yvonne

Ennour-Idrissi, Kaoutar

Enokida, Tomohiro

Enomoto, Masaru

Enose-Akahata, Yoshimi

Enrico, Gringeri

Entz-Werle, Natacha

Eoli, Marica

Er, Tze-Kiong

Eravuchira, Pinkie J.

Erben, Philipp

Erdmann, Joris I.

Eri, S. Srivatsan

Eric, Espinosa

Ericson, Marica B.

Ericson, Marna E.

Eriksson Steigen, Sonja

Erneux, Christophe

Ernst, Corinna

Erokhin, Maksim

Erovic, Boban M.

Escargueil, Alexandre

Escolà-Gil, Joan Carles

Escorcia, Freddy E.

Escors, David

Escudier, Jean-Marc

Eso, Yuji

Espel, Enric

Esposito, Maria Teresa

Esposito, Maria Valeria

Esposito, Roberto
Estanyol, Josep Maria

Esteves Da Silva, Joaquim C. G.

Estévez, Laura García

Estrada, Marta F.

Eu, Peter

Eufemi, Margherita

Éva, Mikó

Evans, D. Gareth

Evans, Gareth R.

Evanson, Nathan K.

Evert, Matthias

Evgin, Laura

Evrard, Solène M.

Ewald, Collin

Eykyn, Thomas

Ezzat, Shereen

Ezzidin, Samer

Fabbri, Francesco

Fabbri, Muller

Fabbrizi, Maria Rita

Faber, Anthony C.

Fabi, Alessandra

Fabiani, Roberto

Fabris, Luca

Fabrizio, Montecucco

Facchiano, Antonio

Facciorusso, Antonio

Facoetti, Angelica

Fadda, Guido

Fadhel, Muhannad N.

Faggiano, Antongiulio

Fagioli, Franca

Fagoonee, Sharmila

Fahraeus, Robin

Faini, Angelo Corso

Faísca, Pedro

Falahat, Rana

Falcioni, Rita

Falcone, Italia

Falk, Stephen

Falzone, Luca

Fan, Meiyun

Fanciulli, Giuseppe

Fandrey, Joachim

Fanetti, Giuseppe

Fang, Qingming

Fang, Wen-Liang

Fanoni, Daniele

Fanti, Alessandro

Fanti, Stefano

Fantini, Sebastian 
Farace, Françoise

Farah, Camile S.

Fardilha, Margarida

Faria-Ramos, Isabel

Farnetani, Francesca

Farolfi, Alberto

Farooqi, Ammad Ahmad

Farquhar-Smith, W. Paul

Farruggia, Piero

Farrugia, Mark K.

Fasano, Morena

Fato, Romana

Fattizzo, Bruno

Faucz, Fabio R.

Faviana, Pinuccia

Faymonville, Marie-Elisabeth

Fazzari, Jennifer

Feda, H. Hamdan

Fedele, Monica

Fedeli, Alessandro

Fehrenbach, Uli

Fei, Miao

Feier, Diana Sorina

Felicetti, Federica

Felidj, Nordin

Feliu, Jaime

Félix, Ana

Felli, Maria Pia

Felsenstein, Matthäus

Feng, Hui

Feng, Wanjuan

Fenton, Tim

Fenyö, David

Feo, Claudio

Feo, Francesco

Feraco, Paola

Ferdek, Paweł

Ferdinandus, Justin

Ferioli, Martina

Fermi, Matteo

Fernandes, Elisabete

Fernandes, Gabriela

Fernandes, Ricardo

Fernández Cabada, Tamara

Fernández Casanova, Lucía

Fernández De Larrea, Carlos

Fernandez, Harvey R.

Fernandez-Botran, Rafael

Fernández-Medarde, Alberto

Fernández-Sáiz, Vanesa

Fernández-Tomé, María Del Carmen
Ferrara, Benedetta

Ferraresi, Alessandra

Ferrari, Daris

Ferrari, Marco

Ferreira, Carolina

Ferreira, Fernando

Ferreira, Rodrigo

Ferrero, Giulio

Ferri, Flaminia

Ferriere, François

Ferroni, Patrizia

Ferrucci, Michela

Ferrucci, Pier Francesco

Feuillard, Jean

Figueiredo, Carlos R.

Figueroa, Angélica

Filbet, Marilène

Filigheddu, Nicoletta

Filipovic, Miodrag

Filippi, Luca

Filippi, Silvia

Filippou, Panagiota $S$.

Filippova, Natalia

Fina, Emanuela

Finazzi, Tobias

Finke, Daniel

Finkielstein, Carla

Fionda, Bruno

Fionda, Cinzia

Fiore, Danilo

Fiorelli, Alfonso

Fiorentini, Carla

Fiorentini, Giammaria

Fiori, Enrico

Fiorica, Francesco

Firczuk, Malgorzata

Fischer, Martin

Fischer, Nicholas W.

Fischer, Nicole

Fischer-Fodor, Eva

Fitch, Margaret

Fitzgerald, David

Fiz, Francesco

Flavin, Richard

Fleitas, Tania

Fliedner, Stephanie

Flores-Montero, Juan

Flórez, Ángeles

Flouriot, Gilles

Folini, Marco

Follo, Matilde Yung 
Fon Tacer, Klementina

Fonseca-Alves, Carlos Eduardo

Fontana, Rosa

Fontanini, Gabriella

Font-Burgada, Joan

Fontenay, Michaela

Foote, Janet A.

Foray, Nicolas

Forciniti, Stefania

Ford, John C.

Forero-Castro, Maribel

Forest, Fabien

Formanek, Martin

Fornasaro, Stefano

Forsare, Carina

Forsberg, Matthew

Försch, Sebastian

Förschler, Annette

Forschner, Andrea

Forster, Michael

Fort, Philippe

Forte, Iris Maria

Fortenberry, Yolanda

Foschini, M. P.

Fossati, Piero

Fox, Joanna L.

Fracasso, Giulio

Fradin, Delphine

Fragomeni, Simona Maria

Fraile-Bethencourt, Eugenia

Fraizer, Gail

Frampton, Adam E.

Franceschini, Gianluca

Francesco, Aniello

Francescone, Ralph

Franchet, Camille

Franchi, Matteo

Francia, Sofia

Francini, Edoardo

Francis, Heather

Francis, Jasmine $\mathrm{H}$.

Francisci, Silvia

Franck, Julien

Franco, Luis

Franco, Pierfrancesco

Francolini, Giulio

Francone, Elisa

Francuz, Tomasz

Franko, Jan

Franses, Joseph W.

Franz, Leonardo
Franzini, Anca

Fraser, Michael

Frassanito, Maria Antonia

Frati, Alessandro

Fratila, Raluca

Fratino, Lucia

Frauenknecht, Katrin

Frazzi, Raffaele

Fredolini, Claudia

Frega, Giorgio

Freitas, Daniela

Freitas, Lucas F.

Fremd, Carlo

Frese, Kristopher

Frew, Ian J.

Frey, Benjamin

Freyer, David R.

Frezzato, Federica

Fribley, Andrew

Fridrichova, Ivana

Frieboes, Hermann B.

Friedl, Anna A.

Friedmann Angeli, José Pedro

Friedrich, Christoph M.

Friedrich, Mirco

Friedrichs, Nicolaus

Friesland, Signe

Friess, Helmut

Frietze, Seth

Frigo, Daniel E.

Frolova, Liliya

Fuchs, Bruno

Fuchs, Hans Friedrich

Fuchs, Herman

Fuchs, Ota

Fueger, Barbara J.

Fujihara, Hisako

Fujii, Shin-ichiro

Fujii, Shoichi

Fujii, Tomomi

Fujii, Youichi

Fujimori, Ko

Fujimura, Atsushi

Fujishima, Fumiyoshi

Fujita, Kazutoshi

Fujita, Naoki

Fujiwara, Masayuki

Fujiwara, Naoto

Fujiwara, Yukio

Fukuoka, Hidenori

Fukushima, Hiroshi 
Fulci, Valerio

Fulton, Lawrence

Fumagalli Romario, Uberto

Fumoto, Shintaro

Funahashi, Akira

Funai, Kazuhito

Furlanetto, Jenny

Furler, Robert L.

Furqan Qadri, Syed

Furtner-Srajer, Julia

Furukawa, Tatsuhiko

Furukawa, Toru

Fusco, Nicola

Fushida, Sachio

Fütterer, Jurgen

Futyma, Konrad

Gabbia, Daniela

Gabrić, Dragana

Gabriel, Emmanuel

Gabrielli, Brian

Gabrielsson, Susanne

Gabryel, Piotr

Gabryś, Dorota

Gaetano, Carlo

Gagat, Maciej

Gagner, Jean-Pierre

Gagos, Sarantis

Gaidano, Gianluca

Gaidano, Valentina

Gaidzik, Verena

Gaikwad, Hanmant

Gainullin, Murat

Gaipl, Udo S.

Gaiser, Timo

Gajek, Arkadiusz

Gajewska, A.

Gajos-Michniewicz, Anna

Galandrini, Ricciarda

Galardy, Paul J.

Galbiati, Silvia

Galetta, Domenico

Galibert, Marie-Dominique

Galindo, Rene

Gallardo, Fernando

Gallego, Óscar S.

Galli, Giulia

Gallivanone, Francesca

Gallo, Gaetano

Gallo, Juan

Gallo, Oreste

Gallo, Salvatore
Gallotta, Valerio

Galvano, Antonio

Gaman, Mihnea-Alexandru

Gambacorta, Maria Antonietta

Gambacorti-Passerini, Carlo

Gambardella, Gennaro

Gamberi, Tania

Gameiro, Steve

Gameiro, Steven F.

Gamradt, Pia

Ganau, Mario

Gangemi, Rosaria

Gangopadhyay, Soumyashree

Ganguly, Anutosh

Gani, Jonathon S.

Ganju, Aditya

Ganser, Arnold

Gao, Allen

Gao, Jingjing

Gao, Shuai

Gaponenko, Vadim

Garattini, Enrico

Garavaglia, Silvia

Garayoa, Mercedes

Garbossa, Diego

García Gutiérrez, José Valentín

Garcia Gutierrez, Lucia

Garcia Hernandez, Juan Luis

García, Juán Fernando

Garcia-Borron, Jose Carlos

García-Escudero, Ramón

García-Flórez, Luis

Garcia-Giralt, Natalia

García-Martínez, Araceli

García-Otín, Ángel Luis

Garcia-Rico, Eduardo

García-Rojo, Marcial

García-Silva, Susana

Gardner, Thomas A.

Garella, Rachele

Garg, Manoj

Garí, Eloi

Gariglio, Marisa

Garje, Rohan

Garofalo, Mariangela

Garrastachu, Puy

Garrè, Maria Luisa

Garrick, David

Garrigue, Philippe

Gartel, Andrei

Gartner, Fátima 
Gasparotto, Daniela

Gasparri, Roberto

Gasparski, Alexander

Gašperov, Nina Milutin

Gassa, Asmae

Gaßler, Nikolaus

Gaston, Kevin

Gatti, Laura

Gatto, Lidia

Gatz, Susanne

Gauchotte, Guillaume

Gaudreau, Pierre Olivier

Gautier, Mathieu

Gavard, Julie

Gavazzi, Francesca

Gavert, Nancy

Gavin, Kara

Gaviraghi, Marco

Gaykalova, Daria A.

Gazouli, Maria

$\mathrm{Ge}, \mathrm{Xin}$

Ge, Yubin

Geary, Sean

Gebauer, Bernhard

Gebauer, Florian

Gebbia, Vittorio

Gefeller, Olaf

Geisberger, Roland

Geisler, Jürgen

Geismann, Claudia

Geldmacher, David

Gelsomino, Francesco

Gener, Petra

Genitori, Lorenzo

Gennari, Alessandra

Genovesi-Ebert, Federica

Gentilini, Oreste D.

Genuardi, Elisa

Geoffrois, Lionnel

Geok Hoon, Lim

Georgakilas, Alexandros

Georgantopoulos, Peter

Georges, Steven

Georgoulias, Vassilis

Geppert, Carol

Gérard, Jéan Pierre

Gergely, Lajos

Gerhard, Markus

Gerloff, Dennis

Germain, Pierre

Germanidis, Georgios
Germini, Diego

Gerner, Christopher

Gerum, Sabine

Geso, Moshi

Gessani, Sandra

Gessi, Stefania

Gessler, Florian

Geuna, Massimo

Gevertz, Jana

Ghanim, Bahil

Ghansah, Tomar

Ghantoji, Shashank S.

Ghazal, Hassan

Ghebeh, Hazem

Gherardin, Nicholas

Gherase, Mihai

Ghiasvand, Reza

Ghiringhelli, François

Ghodoussipour, Saum B.

Ghosh, Arijit

Ghosh, Paramita M.

Ghosh, Sourav

Ghosh, Sunita

Giaccone, Luisa

Giacomelli, Luca

Giacomelli, Michael

Giacomini, Patrizio

Giacopuzzi, Simone

Giampieri, Riccardo

Gianelli, Umberto

Giannella, Luca

Giannetta, Elisa

Giannì, Aldo Bruno

Giannouli, Stavroula

Giatsidis, Giorgio

Gibbons, Andrea

Gibbs, Bernhard

Gibbs, Peter

Gibson, Spencer

Giebel, Sebastian

Giesen, Nicola

Gil, Justyna

Gilbert, Duncan C.

Gilhuijs, Kenneth

Gill, Harinder

Gilles, Christine

Gillet, Germain

Gillet, Jean Pierre

Gillinder, Kevin

Ginesu, Giorgio

Giommoni, Elisa 
Giordano, Antonio

Giordano, Guido

Giorgakis, Emmanouil

Giorgini, Elisabetta

Giovannetti, Elisa

Giovannini, Giada

Giovarelli, Mirella

Giraldo, Pilar

Girault, Alban

Girelli, Roberto

Giri, Anil K.

Girotto, Stefania

Gisselbrecht, Christian

Gitto, Sarah B.

Giudice, Valentina

Giuffrè, Mauro

Giurisato, Emanuele

Giustacchini, Alice

Gjorgjieva, Monika

Gkolfakis, Paraskevas

Glantzounis, Georgios K.

Glass, Jon

Glatting, Gerhard

Gleeson, Noreen

Gliwiński, Mateusz

Glossop, Neil

Glover, Anthony

Gnanasundram, Sivakumar Vadivel

Godet, Yann

Godlewska, Marlena

Godlewski, Janusz

Godos, Justyna

Goel, Sanjay

Goemans, Bianca

Gogulamudi, Venkateswara Reddy

Gogvadze, Vladimir

Gokhale, Prafulla

Gokulan, Ravindran Caspa

Goldman, Radoslav

Golebiewska, Anna

Golias, Tereza

Golla, Upendar

Göllner, Stefanie

Golubnitschaja, Olga

Golubovskaya, Vita

Golumbeanu, Monica

Gomarasca, Marta

Gomes, Catarina

Gomes-da-Silva, Lígia C.

Gómez Cerezo, Jorge Francisco

Gómez De Cedrón, Marta
Gomez Gomez, Enrique

Gomez-Sanchez, Celso

Gomez-Serranillos, Marta Sanchez

Gonçalves, Ana Cristina

Gonçalves, Bruno

Gongora, Celine

Goni, Felix

Gonnella, Roberta

Gonsalves, Wilson I.

González, Álvaro

Gonzalez, Antonio

Gonzalez, Miguel Toro

Gonzalez, Raul S.

González, Segundo

González, Sonia

González-Gascón Y Marín, Isabel

González-Sarmiento, Rogelio

González-Vallinas, Margarita

Goodwin, Craig M.

Goossens, Nicolas

Gopal, Keshav

Gopalakrishnapillai, Anilkumar

Gorchakov, Andrey A.

Gorczynski, Reginald

Gordeeva, Olga

Gore, Mitchell R.

Gorgulu, Kivanc

Goričar, Katja

Gorji, Ali

Gorrell, Mark

Gorynski, Krzysztof

Goss, Alastair N.

Goswami, Sandeep

Goto, Akiteru

Goto, Taichiro

Goto, Yusuke

Gotthardt, Martin

Goulet, Eric

Goussia, Anna C.

Gout, Johann

Gouttefangea, Cecile

Gozzini, Antonella

Grabarek, Beniamin Oskar

Grabbert, Markus Tobias

Grabowska, Anna M.

Gracia, Jose Luis Perez

Graczyk-Jarzynka, Agnieszka

Grade, Marian

Gradissimo, Ana

Graesser, Friedrich

Graf, Solomon A. 
Graff, Pierre

Graham, Charlotte

Graham, Jeffrey

Grana, Chiara Maria

Granados-Principal, Sergio M.

Granato, Marisa

Granberg, Dan

Grand, Roger

Grande, Fedora

Grander, Christoph

Granito, Alessandro

Grasa, Laura

Graslund, Torbjorn

Grass, G. Daniel

Grassi, Gabriele

Grauslund, Morten

Graves, Lee M.

Gravis, Gwenaelle

Gray, Steven G.

Grazi, Gian Luca

Graziani, Grazia

Green, Michael

Gregorieff, Alex

Greidanus, Michiel A

Greither, Thomas

Griebenow, Kai

Griffin, Robert J.

Grignani, Giovanni

Griguer, Corinne E.

Griñán-Lisón, Carmen

Grippo, Paul J.

Grisanti, Salvatore

Grit, Jamie L.

Grodzik, Marta

Groma, Valerija

Groner, Bernd

Gronwald, Jacek

Grösch, Sabine

Grose, Derek

Gross, Julia Christina

Gross, Stephane R.

Großhans, Jörg

Grosu, Anca-Ligia

Grötzinger, Carsten

Grumati, Paolo

Grumolato, Luca

Grüner, Florian

Grüning, Björn A

Gruppo, Mario

Grzegrzółka, Jędrzej

Grzybowska, Ewa A.
Grzywa, Tomasz

$\mathrm{Gu}$, Bin

Gu, Xiaolian

Gu, Xinbin

Guadagni, Fiorella

Guadagni, Stefano

Guan, Fada

Guan, Yihong

Guarino, Andrea Maria

Guarino, Maria

Guda, Maheedhara R.

Gudey, Shyam Kumar

Gueresir, Erdem

Gueron, Geraldine

Guerra, Carmen

Guerra, Flora

Guerra, Jessica

Guerra-Librero, Ana

Guerriero, Ilaria

Guèye, Paul

Gugasyan, Raffi

Guglielmo, Priscilla

Gugnoni, Mila

Guh, Jih-Hwa

Guha, Prasun

Guha, Udayan

Guida, Michele

Guidi, Patrizia

Guidoboni, Massimo

Guidoni, Laura

Guillaud, Martial

Guillaume, Thierry A.

Guillermet Guibert, Julie

Guirouilh-Barbat, Josee

Gujar, Hemant

Gulati, Rohit

Guldvik, Ingrid Jenny

Gulla, Aiste

Gulve, Nitish

Gulyaeva, Lyudmila F.

Gunaje, Jayarama

Gundlach, Jan-Paul

Guo, Lilong

Guo, Siqi

Guo, Zengli

Guo, Zong Sheng

Guolo, Fabio

Gupta, Ajay

Gupta, Mohit

Gupta, Ramesh C.

Gupta, Rishein 
Gupta, Romi

Gupta, Sanjay

Gupta, Seema

Gupta, Vijayalaxmi G.

Gupta, Vineet K.

Gupta, Yash

Gurnari, Carmelo

Gurney, Howard

Gursky, Jan

Gust, Kilian Martin

Guth, Amanda

Gutierrez, Linda S.

Gutiérrez, María Laura

Gutierrez-Aguilar, Manuel

Guttery, David S.

Gutzmer, Ralf

Guyon, Laurent

Guyot, Boris

Guyot, Erwan

Gyllensten, Ulf

Gyulai, Rolland

$\mathrm{Ha}$, Jeonghoon

Ha, Min-woo

Ha, Yun-Sok

Haag, Friedrich

Haag, Mathias

Habibollahi, Peiman

Hacker, Neville

Hacker, Ulrich

Hackl, Christina

Hackl, Hubert

Haderlein, Marlen

Hadidi, Samer Al

Hadler-Olsen, Elin Synnøve

Haffner, Michael

Häfner, Norman

Hagemann, Anja I. H.

Hägerling, René

Haglund, Felix

Hahn, Oliver

Hahne, Jens

Hahne, Michael

Haier, Jörg

Hájek, Roman

Haj-Hosseini, Neda

Halaban, Ruth

Hałas-Wiśniewska, Marta

Haldorsen, Ingfrid S.

Halicek, Martin T.

Halkett, Georgia

Hall, Per F. L.
Hallaert, Giorgio

Hallden, Gunnel

Ham, Won-Sik

Hamada, Shin

Hamamoto, Ryuji

Hamanishi, Junzo

Hamburger, Anne W.

Hammad, Mohamed Adel

Hammer, Elke

Hammill, Jared

Hammoud, Ghassan M.

Hamzeh-Cognasse, Hind

Han, Chiali

Han, Dong

Han, Hongwei

Han, Ilkyu

Han, Ki-Ho

Han, Yanhui

Han, Youngmin

Han, Yuyan

Hanafy, Nemany Abdelhamid Nemany

Hancock, Sarah

Handa, Hiroshi

Hanin, Leonid

Hanke, Tomas

Hanker, Ariella

Hanks, Brent A.

Hanley, Chirstopher

Hannoush, Zeina Carolina

Hanoun, Maher

Hansen, Carsten Gram

Hansen, Charlotte Toftmann

Hansen, Hinrich P.

Hansen, Jakob Werner

Hansen, Torben Frøstrup

Happel, Christian

Hapuarachchige, Sudath

Haque, Muhammad R.

Haque, Reina

Harada, Mamoru

Harada, Takeshi

Harada, Toshiyuki

Harashima, Nanae

Harbison, R. Alex

Harbottle, Richard

Harder, Anja

Hardt, Julia

Hardt, Olaf

Hardtke-Wolenski, Matthias

Hareendran, Sangeetha

Harisinghani, Mukesh 
Harling, Leanne

Harrell, J. Chuck

Harrer, Dennis

Harrington, Brittney

Harris, Brent T.

Harrison, David J.

Härteis, Silke

Hartl, Dana M.

Hartley, Andrew

Hartman, Johan

Hartman, Mariusz

Hartmann, Daniela

Hartmann-Johnsen, Olaf Johan

Hartsough, Edward

Harvey, James R.

Hasanzadeh Kafshgari, Morteza

Hasegawa, Shinya

Hasegawa, Sumitaka

Hashida, Noriyasu

Hashimoto, Daisuke

Hashimoto, Kazuhiko

Hasipek, Metis

Hasoňová, Lucie

Hassan, Mohamed

Hassan, Sherif T. S.

Hasse, Sybille

Hasselgren, Per Olof

Hata, Masaharu

Hatakeyama, Hiroto

Hatano, Koji

Hatano, Yuichiro

Hato, Tai

Hattori, Naoko

Hau, Peter

Haug, Alexander

Haugk, Beate

Haugnes, Hege Sagstuen

Haumaitre, Cécile

Hauptmann, Steffen

Hauser, Hansjoerg

Hauser, Peter

Haveman, Lianne M.

Hawkridge, Adam M.

Hay, Jennifer

Hayasaka, Haruko

Hayashi, Katsuhiro

Hayashida, Kenji

Haybaeck, Johannes

Haynes, Jennifer

Hayward, Mary-Kate

Hazama, Shyouichi
Hazawa, Masaharu

Hazlehurst, Lori A.

$\mathrm{He}$, Jin

He, Yazhou

He, Yukai

Heakal, Yasser

Healey, Gareth

Hebeda, Konnie M.

Heckl, Dirk

Hee-chul, Park

Heeke, Simon

Heer, Rakesh

Hegde, Bindu

Heger, Ulrike

Heiblig, Mael

Heigener, David F.

Heinzmann, David

Heitmann, Jonas S.

Hejnar, Jiri

Helen Jane, Boyle

Heller, Gerwin

Heller, Richard

Helms, Volkhard

Henderson-Jackson, Evita

Hendriks, Manya

Heng, Henry $\mathrm{H}$.

Héninger, Erika

Henkenberens, Christoph

Hennig, Ewa

Hennkens, Heather M.

Henry, Claire

Henry, Ryan A.

Henry, Yves

Henry-Amar, Michel C.

Henson, Jeremy

Heras, Sara M.

Herbig, Maik

Herbreteau, Guillaume

Herbst, Allen

Herbst, Andreas

Hercbergs, Aleck

Herfarth, Klaus K.

Hermann, R. M.

Hermouet, Sylvie

Hernandez-Alcoceba, Ruben

Hernãndez-Bronchud, Miguel H.

Hernández-Rivas, José-Ángel

Hernández-Sánchez, María

Herndler-Brandstetter, Dietmar

Hernes, Eivor $\mathrm{H}$.

Herr, Ingrid 
Herraez, Elisa

Herrera, Blanca

Herreros-Pomares, Alejandro

Hersey, Peter

Herskind, Carsten

Hervé, Técher

Hervouet, Eric

Herwig-Carl, Martina C.

Hester, Joanna

Hettie, Kenneth

Heumann, Rolf

Heuskin, Anne-Catherine

Hicks, Rodney

Hicks, Wesley L.

Hickson, Ian

Hier, Michael

Higano, Celestia S.

Higurashi, Takuma

Hijiya, Nobuko

Hilakivi-Clarke, Leena

Hill, Mark

Hill, Michelle

Hill, Rebecca

Hillmer, Axel M.

Hiltbrunner, Stefanie

Himoto, Takashi

Hindié, Elif

Hingorani, Dina V.

Hinkley, Leighton

Hirano, Hidekazu

Hiraoka, Nobuyoshi

Hirata, Hiroaki

Hirata, Kenji

Hirohashi, Yoshihiko

Hironaka, Shuichi

Hirose, Yoshinobu

Hitt, Ricardo

Hiwasa, Takaki

Hjerpe, Anders

Ho, Cheng-Maw

Ho, Chengying

Ho, Ivy A. W.

Ho, James Chung-man

Ho, Sheng-Yow

Hobbs, Gabriela Soriano

Hochwald, Steven N.

Hodny, Zdenek

Hodson, Daniel

Hoefler, Gerald

Hoffmann, Caroline

Hoffmann, Franziska
Hoffmann, Markus

Hofmann, Peter

Hogan, Andrew E.

Hohaus, Stefan

Hoheisel, Jörg D.

Hohenberger, Peter

Hohenegger, Martin

Hohmann, Tim

Höhne, Julius

Hojan, Katarzyna

Hojjat-Farsangi, Mohammad

Hokamp, Nils GroBe

Holmager, Pernille

Holmberg, Carina I.

Hölscher, Tobias

Höltke, Carsten

Holubekova, Veronika

Holubova, Monika

Holzmann, Klaus

Hombach, Andreas A.

Hombach-Klonisch, Sabine

Honda, Tomoyuki

Honegger, Juergen

Honeywell, Richard J.

Hong, Andrew L.

Hong, Changwan

Hong, Hyun Sook

Hong, Min Hee

Honoré, Bent

Hooda, Jagmohan

Hoogwater, Frederik

Hooper, Douglas C.

Hooper, John D.

Höpfner, Michael

Höppner, Jens

Horak, Peter

Horeweg, Nanda

Horger, Marius

Horinouchi, Hidehito

Horn, Lucas A.

Hornak, Joseph P.

Hornsveld, Marten

Horsky, Jiři

Horstmann, Marcus

Horvath, Anelia

Horvath, Robert

Hoshino, Daisuke

Hoskins, Clare

Hossain, Kaniz Fatima Binte

Hosui, Atsushi

Hottinger, Andreas F. 
Hou, Hsin-An

Hou, Jue

Houben, Roland

Houdek, Matthew

House, Carrie

House, Imran

Houshmand, Mohammad

Houtman, Jon

Houvenaeghel, G.

Hoving, Eelco

Hoy, Andrew

Hrstka, Roman

Hrvat, Antonio

Hsiao, Hui-Hua

Hsiao, Kuei-Yang

Hsiao, Sheng-Mou

Hsiao, Yungchin

Hsieh, Chia-Chien

Hsieh, Chien-Ming

Hsieh, Ming-Ching

Hsieh, Ming-Li

Hsieh, Pei-Ling

Hsieh, Tsung-Han

Hsu, Feiting

Hsu, Fei-Ting

Hsu, Kai-Wen

Hsu, Keng-Fu

Hsu, Ping-Chih

Hsu, Shih-Hsien

Hsu, Shu-Ching

Hsu, Sigmund

Hsu, Tsung-I

Hsu, Wen-Ming

Hsu, Ya-Ling

Hsu, Yi-Chiung

$\mathrm{Hu}$, Baoli

$\mathrm{Hu}$, Jack

$\mathrm{Hu}$, Jennifer

$\mathrm{Hu}$, Taobo

$\mathrm{Hu}$, Xiaowen

Huang, Haiqiu

Huang, Hao

Huang, Huang Chiao

Huang, Huey-Chun

Huang, Huocong

Huang, Junfeng

Huang, Kai Wen

Huang, Paul Li-Hao

Huang, Shiang-Fu

Huang, Shin-Ming

Huang, Shu-Pin
Huang, Szu-Wei

Huang, Wen-Chin

Huang, Xiaohu

Huang, Xudong

Huang, Yi-Hsiang

Huang, Ying

HuangFu, Wei-Chun

Huber, Magdalena

Huber, Peter

Hübner, Ralph

Huda, Nazmul

Hudler, Petra

Huebbers, Christian U.

Huebner, Hanna

Huen, Michael Shing Yan

Hughes, Kevin

Hughes, Michael

Hugo, Willy

Huidobro, Covadonga

Hulin, Anne

Hulleman, Esther

Hulshof, Maarten C. C. M.

Humphries, Jonathan D.

Hüneburg, Robert

Hung, Chin-Chuan

Hung, Ming-Szu

Hung, Shih-Kai

Hung, Yao-Ching

Hung, Yin Pun

Hunter, Anthony Michael

Hunter, Beth

Hunter, Zachary

Huntington, Nicholas David

Huntosova, Veronika

Hupe, Marie C.

Hur, Hoon

Hurbin, Amandine

Husnjak, Koraljka

Huss, Ralf

Hussaini, Haizal

Hutchinson, John N.

Huvenne, Wouter

Hwang, Sung Il

Hwangbo, Cheol

Hwu, Yueh-Juen

Hyun, Sang-Hwan

Iaccino, Enrico

Iachina, Maria K.

Iacobucci, Ilaria

Iacovelli, Roberto

Iannitto, Emilio 
Iatí, Giuseppe

Iavarone, Federica

Ibáñez-Costa, Alejandro

Ibbs, Matthew

Ibrahim, Abdalla

Ibrahim, Sherif Abdelaziz

Idoate, Miguel

Ierardi, Enzo

Igaz, Peter

Iglesias, Emilia

Iglesias-Ara, Ainhoa

Ignatius, Myron

Ignee, Andre

Ihle, Andreas

Ikematsu, Hiroaki

Ikesue, Hiroaki

Ikonomopoulou, Maria

Illei, Peter B.

Illert, Lena

Illing, Patricia T.

Ilonen, Ilkka K.

Imai, Chihaya

Imai, Yasuo

Imamura, Masahiro

Imanirad, Iman

Imig, Jochen

In 't Veld, Sjors G. J. G.

In 't Zandt, Rene

Inama, Marco

Inamdar, Sumant

Inderberg, Else Marit

Indini, Alice

Indraccolo, Stefano

Infante, Mar

Inga, Alberto

Ingram, Nicola

Ingrosso, Gianluca

Inno, Alessandro

Innominato, Pasquale

Inoue, Takahiro

Inyushin, Mikhail Y.

Ioffe, Yevgeniya

Iommelli, Francesca

Irace, Carlo

Irini, Evnouchidou

Iriuchishima, Hirono

Irjala, Heikki

Isayama, Hiroyuki

Isemura, Mamoru

Ishiai, Masamichi

Ishibashi, Toyotaka
Ishida, Fumihiro

Ishida, Tadao

Ishido, Keinosuke

Ishihara, Katsuhiko

Ishihara, Seiichiro

Ishikawa, Eiichi

Ishikawa, Eri

Ishikawa, Hitoshi

Ishikawa, Mitsuya

Ishikawa, Toru

Ishitsuka, Yousuke

Ishiwata-Endo, Hiroko

Ishiyama, Hiromichi

Ishizawa, Takeaki

Ishizuka, Mitsuru

Islam, Shariful

Isohashi, Fumiaki

Isola, Miriam

Issels, Rolf Dieter

Itabaiana, Ivaldo

Itabashi, Michio

Itälä-Remes, Maija

Italiano, Antoine

Itami, Jun

Itamochi, Hiroaki

Itkonen, Harri

Ito, Hiromichi

Ito, Takamichi

Ituarte, Philip

Iwamoto, Hideki

Iwamoto, Shotaro

Iwanicki, Marcin

Iwaszkiewicz-Grzes, Dorota

Iwata, Takehiro

Izdebska, Magdalena

Izzotti, Alberto

Jabłońska, Beata

Jackson, James

Jackson, Michael A.

Jacob, Sheeba

Jacobsen, Anders

Jacqmin, Hugues

Jacquelot, Nicolas

Jafari, Nancy Najmeh

Jäger, Roland

Jaggi, Meena

Jagiełło-Gruszfeld, Agnieszka

Jahangiri, Leila

Jahn, Arne

Jain, Suneil

Jaiswal, Ashvin R. 
Jaiswal, Deepika

Jakopovic, Marko

Jakšić, Daniela

Jakubek, Milan

Jakubowska, Monika A.

Jalkanen, Sirpa

Jamar, François

James, Claire D.

Jamitzky, Silke

Jan, Ming-Shiou

Janczar, Szymon

Janda, Elzbieta

Jandrot-Perrus, Martine

Janecki, Marcin

Jané-Salas, Enric

Jang, Hyun-jong

Jang, Sung-Wuk

Janíková, Andrea

Janikowska, Grażyna

Jankovic, Momcilo

Janmaat, Vincent $\mathrm{T}$.

Jänne, Olli A.

Janody, Florence

Janowski, Miroslaw

Jansen, C. A. (Christine)

Jansen, Gerrit

Jansen, Yanina Jeanne Leona.

Janssen, Emiel

Janssen, Nicole

Jansson, Patric J.

Januszkiewicz-Lewandowska, Danuta

Jarząb, Barbara

Jassim, Sarmad H.

Jaukovic, Aleksandra

Javadian, Pouya

Javaheri, Tahereh

Jaworek- Korjakowska, Joanna

Jaworek, Jolanta

Jayaprakash, Priyamvada

Jaye, David L.

Jazayeri, Seyed Behzad

Jazirehi, Ali R.

Jeffers, Kate D.

Jelinek, Tomas

Jenkins, Russell W.

Jennifer, Sun

Jeon, Min Ji

Jeong, Kyoung-sook

Jeong, Seri

Jeong, Seung-Yong

Jeschke, Udo
Jesenko, Tanja

Jesse, Christopher M.

Jeucken, Aike

Ji, Peng

Ji, Zhicheng

Jian, Xing

Jiang, Dawei

Jiang, Li

Jiang, Lin-Hua

Jiang, Shih Sheng

Jiang, Xiaodong

Jiang, Yong

Jiménez-González, Gema

Jiménez-Luna, Cristina

Jimi, Eijiro

Jin, Dong-Hoon

Jin, Jiefu

Jin, Weilin

Jin, Zhaohui

Jochmanová, Ivana

Jochum, Christoph

Joh, Richard Inho

Johanns, Tanner M.

Johansson, Ulrika

Johnen, Georg

Johnson, Christopher M.

Johnson, James E.

Johnson, Jeff

Johnson, Jennifer M.

Johnson, Nathalie A.

Jöhrens, Korinna

Jolly, Mohit Kumar

Jomaa, Mona K.

Jonassen, Christine

Jones, Bleddyn

Jones, Kenneth

Jönsson, Jenny-Maria

Jordan, Bénédicte F.

Jørgensen, Heather

Joseph, Chitra

Joshi, Gaurav

Joshi, Molishree Umesh

Joshi, Sonali

Josse, Claire

Jost, Edgar

Jou, Yuh-shan

Joura, Elmar A.

Jourdain, Alexis

Jozwik, Agnieszka

Jozwik, Marcin

$\mathrm{Ju}$, Xiangqun 
Jud, Sebastian M.

Juette, Hendrik

Juhasz, Kata

Juhlin, Christofer

Julián, Esther

Julià-Sapé, Margarida

Jung, Chan Kwon

Jung, Ji Hoon

Jung, Joohee

Jung, Keehoon

Jung, Klaus

Jung, Yuh-Seog

Junker, Kerstin

Juozaitytė, Elona

Jurj, Ancuța Maria

Jurkovicova, Dana

Kabe, Yasuaki

Kabir, Mohammad Faujul

Kabziński, Jacek

Kacew, Alec J.

Kaczmarek, Radoslaw

Kae, Nakamura

Kaesmann, Lukas

Kaewkhaw, Rossukon

Kagamu, Hiroshi

Kagey, Jacob

Kahlert, Ulf

Kaigorodova, Evgeniya

Kaira, Kyoichi

Kairemo, Kalevi

Kajihara, Mikio

Kajimoto, Shinji

Kajtár, Béla

Kakehashi, Anna

Kakizawa, Nao

Kakkassery, Vinodh

Kalainayakan, Sarada Preeta

Kalamarides, Michel

Kalasauskas, Darius

Kalathil, Suresh

Kalathiya, Umesh

Kalayda, Ganna

Kalb, Reinhard

Kalinova, Marketa

Kalirai, Helen

Kaliszewski, Krzysztof

Kalita-de Croft, Priyakshi

Kaljunen, Heidi

Kállay, Enikö

Kallendrusch, Sonja

Kaller, Markus
Kallergi, Galatea

Kallioniemi, Elisa

Kalthoff, Holger

Kaltsas, Gregory

Kaltschmidt, Barbara

Kałuża, Anna

Kamada, Yoshihiro

Kamensek, Urska

Kameyama, Kaori

Kamijo, Takehiko

Kamimura, Hiroteru

Kamimura, Kenya

Kaminska, Bozena

Kammala, Ananth Kumar

Kampen, Kim

Kamphues, Carsten

Kamran, Mohammad

Kanagasabai, Thanigaivelan

Kanapin, Alexander

Kanaujiya, Jitendra

Kanayama, Koji

Kanda, Tatsuo

Kandasamy, Palanivel

Kandpal, Manoj

Kaneda, Atsushi

Kaneda, Masahiro

Kanegasaki, Shiro

Kaneko, Syuzo

Kanellakis, Nikolaos I.

Kanemura, Nobuhiro

Kanemura, Yonehiro

Kang, Chang Moo

Kang, ChangSun

Kang, Dongchul

Kang, Dong-Woo

Kang, Eunju

Kang, Jae Seung

Kang, Koo Jeong

Kang, Kyuho

Kang, Min Kyu

Kang, Tae Hyun

Kang, Yu

Kanginakudru, Sriramana

Kanis, Margaux Jenna

Kant, Shiva

Kao, Chien-Chang

Kao, Shao-Hsuan

Kaochar, Salma

Kapischke, Matthias

Kaplan, David E.

Kaplan, Michael J. 
Kappler, Matthias

Kapral, Małgorzata

Karacosta, Loukia G.

Karakaidos, Panagiotis

Karakas, Cansu

Karakatsanis, Andreas

Karamouzis, Michalis

Karantanos, Theodoros

Karavitakis, Markos

Karin, Nathan

Karnati, Srikanth

Karousou, Eugenia

Karow, Axel

Karpathiou, Georgia

Karpel-Massler, Georg

Karpiński, Tomasz M.

Karpov, Dmitry

Karras, Panagiotis

Karreman, Matthia A.

Karsak, Meliha

Karthikeyan, Mythreye

Kartsonaki, Christiana

Kasahara, Noriyuki

Kashima, Shin

Kashino, Genrou

Kashiwagi, Shinichiro

Kashofer, Karl

Kasiotis, Kostas

Kasman, Laura

Kasperkiewicz, Paulina

Kasprzak, Aldona

Kasymjanova, Goulnar

Kasza, Aneta

Kasztelan-Szczerbińska, Beata

Kataoka, Naoyuki

Kataoka, Tatsuki

Katapodi, Maria C.

Katarzyna, Malarz

Katayama, Maria Lucia Hirata

Katira, Parag

Kato, Katsuhiko

Kato, Ken

Kato, Takamitsu A.

Kato, Tomoyasu

Katsanis, Emmanuel

Katsila, Theodora

Katsuta, Eriko

Katzenellenbogen, Rachel

Kauppila, Joonas H.

Kaur, Kawaljit

Kaur, Sukhbir
Kaur, Tanpreet

Kaushik, Garima

Kaushik, Nagendra Kumar

Kawabata, Hideaki

Kawabata-Iwakawa, Reika

Kawagishi, Naoki

Kawaguchi, Nanako

Kawai, Manabu

Kawai, Tatsuya

Kawaida, Miho

Kawale, Ajinkya

Kawano, Kouichiro

Kawaoka, Tomokazu

Kawashima, Mariko

Kawauchi, Keiko

Kazanietz, Marcelo G.

Kazlauskas, Arunas

Kazuo, Yashima

Ke, Yiyu

Ke, Youqiang

Keek, Simon

Keereweer, Stijn

Kefleyesus, Amaniel

Keisari, Yona

Keklikoglou, Ioanna

Keller, Jonathan R.

Kellner, Christian

Kelly, Dervla

Kemeny, Nancy E.

Kempiński, Radosław

Kenawy, Nihal

Kendall, Tim

Kennedy, Michael A.

Képénékian, Vahan

Kępka, Lucyna

Kerbage, Yohan

Kerkhofs, Thomas

Kerkmeijer, Linda

Kern, Izidor

Kern, Peter

Kerns, Jemma G.

Kersting, Stephan

Kesh, Kousik

Keshari, Sunita

Kesharwani, Siddharth S.

Kessel, Katharina

Kessler, Torsten

Keszler, Gergely

Ketola, Kirsi

Ketteler, Petra

Kettenbach, Joachim 
Ketter, Ralf

Khairnar, Vishal S.

Khakoo, Shelize

Khammanivong, Ali

Khan, Firdos Alam

Khan, Imtiaz

Khan, Mohd Sajid

Khan, Niloufer

Khan, Shahanshah

Khan, Shaheen

Khare, Priyanka

Khazal, Sajad

Khilov, Aleksandr V.

Khivansara, Vishal

Khochenkov, Dmitry

Kholová, Ivana

Khoury, Thaer

Khurana, Namrata

Kida, Yasuyuki S.

Kiechle, Marion

Kieler, Markus

Kiesel, Ludwig

Kiesewetter, Barbara

Kiewisz, Jolanta

Kikuyama, Masataka

Kim, Byung Soo

Kim, Chan Gyoo

Kim, Dongin

Kim, Euishin Edmund

Kim, Eun-Hee

Kim, Hee Seung

Kim, Hee Sung

Kim, Hongbeom

Kim, Hun Sik

Kim, Hyeong-Geug

Kim, Hyungjin

Kim, Ik-Hwan

Kim, Jae-hwan

Kim, Jaesung

Kim, Ja-Eun

Kim, Jin Hyoung

Kim, Jin-Hong

Kim, Jin-hwi

Kim, Jong Man

Kim, Kibeom

Kim, Kyoung Sub

Kim, Kyunggon

Kim, Kyunghwan

Kim, Min-Sik

Kim, Raymond $\mathrm{H}$.

Kim, Ryong Nam
Kim, Ryungsa

Kim, Sang Wun

Kim, Sang-We

Kim, Seok-Jun

Kim, Soochi

Kim, Su Zy

Kim, Sun Hwa

Kim, Sung Eun

Kim, Sung Joon

Kim, Sung-Hoon

Kim, Tae Min

Kim, Tae-Don

Kim, Usha

Kim, Woojin

Kim, Yeul Hong

Kim, Yong-Mi

Kimble-Hill, Ann

Kimura, Shoji

Kimura, Yasutoshi

Kimura, Yoshiharu

Kindt-Dunjko, Alida

King, Chih-Yen

King, Renee

Kingsley, Karl

Kinj, Rémy

Kino, Katsuhito

Kinoshita, Tomomari

Kioi, Mitomu

Kiouptsi, Klytaimnistra

Kirac, Iva

Kiran, Sonia

Kirken, Robert A.

Kishore Sakharkar, Meena

Kiss, Norbert

Kistenev, Yurii

Kitadate, Akihiro

Kitagawa, Seiichi

Kitaichi, Kiyoyuki

Kitano, Masayuki

Kitt, Jay

Kittel, Agnes

Kiyohara, Eiji

Klampatsa, Astero

Klapdor, Rüdiger

Kleeff, Jörg

Kleibl, Zdenek

Klein, Alexander

Klein, Sebastian

Kleindienst, Andrea

Kleinman, Hynda

Klencki, Mariusz 
Klener, Pavel

Klepinin, Aleksandr

Klepsch, Victoria

Klimaszewska-Wiśniewska, Anna

Klimczak, Aleksandra

Klimkowska, Monika

Klinakis, Apostolos

Klinge, Carolyn M.

Klink, Magdalena

Klinkhammer-Schalke, Monika

Kluger, Harriet M.

Klyui, Nickolai I.

Knapper, Steven

Knauf, Jeffrey

Knebel, Carolin

Knez, Jure

Knopfova, Lucia

Knuschke, Torben

Ko, Beomseok

Ko, Jiunn- Liang

Ko, Myunggon

Ko, Na-Re

Ko, Ryo

Kobaek-Larsen, Morten

Kobayashi, Hiroshi

Kobayashi, Makoto

Kobayashi, Noritoshi

Kobeissy, Firas

Koch, Oliver

Kochat, Veena

Kocian, Roman

Kodama, Takahiro

Koehl, Gudrun

Koehl, Gudrun E.

Koelblinger, Peter

Kofler, Lukas

Koga, Fumitaka

Koga, Yoshikatsu

Koh, David

Koh, Yong Qin

Koh, Young Wha

Kohyama, Atsushi

Koivunen, Jarkko

Koivusalo, Antti Ilmari

Kok, Chung Hoow

Kokkonda, Praveen

Kolberg, Hans-Christian

Kolekar, Pandurang

Kolesar, Jill Marie

Koliakos, George

Koljonen, Virve
Kolkova, Zuzana

Kolligs, Frank T.

Kolomeǐchuk, Sergey

Kolot, Mikhail N.

Kolovskaya, Olga

Koma, Yuichiro

Komine, Mayumi

Komohara, Yoshihiro

Komorowski, Andrzej

Komune, Noritaka

Kondo, Motonari

Kondo, Tadakazu

Kondrashova, Olga

Kong, Li Ren

Kong, Moonkyoo

Kong, Siu-Kai

Konishi, Hiroaki

Konner, Jason A.

Konoike, Naho

Konsman, Jan Pieter

Konstantakou, Eumorphia G.

Konstantinos, Tepetes

Kontogeorgos, George

Kontogianni, Georgia

Kontos, Christos K.

Koo, Kendrick

Koo, Kevin M.

Koob, Sebastian

Kook, Myeong-Cheryl

Koontz, Bridget F.

Kopaczyńska, Marta

Kopec, Monika

Kopecký, Jindrich

Kopp, Reinhard

Kopp, Sascha

Koppisch, Dorothea

Kopsida, Maria

Korac Prlic, Jelena

Korać, Aleksandra

Korc, Murray

Kordulewska, Natalia

Korf, Horst Werner

Koritzinsky, Marianne

Kornek, Miroslaw

Korolkova, Olga Y.

Korsching, Eberhard

Korshoej, Anders Rosendal

Kosan, Christian

Koseła-Paterczyk, Hanna M.

Koshel, Elena

Kostara, Christina E. 
Köster, Claus

Kostev, Karel

Kostic, Tatjana S.

Kostopoulos, Spiros A.

Kosuga, Toshiyuki

Kosuge, Yasuhiro

Kot, Karolina

Kota, Satya

Kotarski, Jan

Kotaška, Karel

Kotecha, Rupesh Rajesh

Kotelevets, Larissa

Koukourakis, Michael I.

Kouokam, J. Calvin

Kouro, Taku

Kouroumalis, Elias A.

Koustas, Evangelos

Kouttab, Nick

Kovács, Attila

Kovács, György

Kovalenko, Elena I.

Kowal, Krzysztof

Kowalik, Artur

Kowalska, Karolina

Kowalski, Christoph

Kowarik, Markus C.

Koyama, Tatsuki

Kozlov, Serguei

Kozłowska, Anna

Kozono, David

Kozovska, Zuzana

Krahn, Thomas

Krainer, Michael W.

Kranc, Wieslawa

Krane, L. Spencer

Kranendonk, Mariëtte E. G.

Kranz, Mathias

Krarup-Hansen, Anders

Krasnodębski, Maciej

Kratzke, Robert Arthur

Krautz, Christian

Krawczyk, Malgorzata

Krazinski, Bartlomiej

Krebs, Markus

Kregždytè, Rima

Kreipe, Hans H.

Kreis, Nina-Naomi

Kreis, Stephanie

Krengli, Marco

Kress, Andrea

Kretov, Dmitry
Krier, François

Krijgsman, Daniëlle

Krishnamachary, Balaji

Krishnan, Shanmugarajan

Kristensen, Gunnar B.

Kritsiligkou, Paraskevi

Kroesen, Michiel

Krokidis, Miltiadis

Król, Sylwia Katarzyna

Kron, Philipp

Kron, Tomas

Kroneis, Thomas

Krönke, Jan

Krug, Sebastian

Krum, Susan A.

Kŕušlin, B,ožo

Kruyt, Frank

Kubo, Nobuteru

Kucinska, Malgorzata

Kudela, Erik

Kuehne, Andre

Kuemmel, Sherko

Kuhl, Christiane K.

Kuhlmann, Jan

Kuhn, Elisabetta

Kühn, Thorsten

Kuittinen, Outi

Kulbacka, Julita

Kulczyńska-Przybik, Agnieszka

Kulemzin, Sergey

Kulesa-Mrowiecka, Małgorzata

Kuliczkowski, Kazimierz

Kulka, Janina

Kulkarni, Dhananjaya

Kumar, A. Pratap

Kumar, Ashish

Kumar, Janani

Kumar, Manish

Kumar, Pradeep

Kumar, Prashant

Kumar, Raj

Kumar, Rajesh

Kumar, Rajiv

Kumar, Ravindra

Kumar, Sandeep

Kumar, Sunil

Kumar, Vijay

Kumarasamy, Vishnu Muthuraj

Kumari, Sharda

Kumari, Snehlata

Kumi-Diaka, James 
Kumta, Shekhar M.

Kunda, Nitesh

Kung, Hank F.

Kung, Hsing-Jien

Kung, Hsiu-Ni

Kunkler, Ian

Kuno, Hirofumi

Kunstman, John

Künzel, Julian

Kuo, Chao-Hung

Kuo, Chih-Hsi Scott

Kuo, Ping-Chung

Kuo, Shu-Jui

Kuo, Sung-Hsin

Kure, Shoko

Kurmasheva, Raushan

Kuroda, Etsushi

Kuroda, Hiroaki

Kuroda, Junya

Kurokawa, Hiromi

Kurokawa, Tomohiro

Kuroyanagi, Hiroya

Kurth, Jens

Kuryk, Lukasz

Kušec, Rajko

Kushekhar, Kushi

Kusior, Anna

Kusumbe, Anjali

Kutasovic, Jamie R.

Kutler, David

Kutny, Matthew

Kutter, Claudia

Kuwahara, Kazuhiko

Kwack, KyuBum

Kwapiszewska, Karina

Kwee, Sandi

Kwok, Hoi-Hin

Kwok, Wai

Kwon, Jaewoo

Kwon, Jung Hyun

Kwon, Seong Young

Kwon, So Hee

Kwong, Joseph

Kypta, Robert

Kyrgios, Ioannis

Kyriakou, Ioanna

Kyriazoglou, Anastasios

L. Balint, Balint

La Forgia, Daniele

La Mantia, Ignazio

La Monica, Silvia
La Rocca, Renato V.

La Vaccara, Vincenzo

La Verde, Giuseppe

La, Ting

Laakmann, Elena

Laboisse, Christian L.

Lacina, Lukas

Lacombe, Jerome

Lacour, Brigitte

Ladekarl, Morten

Ladetto, Marco

Laengle, Johannes

Lafont, Elodie

Laganà, Antonio Simone

Lagopati, Nefeli

Lai, Andrew

Lai, Catherine

Lai, Ching Lung

Lai, Chyong-Huey

Lai, Feipei

Lai, James J.

Lai, Lu-Han

Lai, Michele

Lai, Ming-Derg

Lai, Raymond

Lai, Yanhao

Laird, Barry

Lajolo, Carlo

Lakota, Jan

Lalk, Michael

Lallena, María José

Lalli, Enzo

Lallous, Nada

Lamarque, Dominique

Lamarre, Eric D.

Lamas Maceiras, Mónica

Lambertini, Martina

Lamfers, Martine

Lammers, Reiner

Lampridis, Savvas

Lan, Yuan-Tzu

Landon, Chelsea Dawn

Landreville, Solange

Landström, Maréne

Landuzzi, Lorena

Lanfrancone, Luisa

Lang, Alexander

Lang, Fengchao

Lang, Peter

Lang, Roland

Langford, Dale 
Languino, Lucia

Lannigan, Deborah

Lanning, Nathan

Lanuti, Paola

Lanza, Francesco

Lanzi, Cinzia

Lanzillotta, Marco

Lapidus, Rena S.

Lapini, Alberto

Lapins, Jan

Lappano, Rosamaria

Laranjo, Mafalda

Lardone, Patricia J.

Lareau, Caleb

Larese, Francesca

Larimer, Benjamin M.

Larionov, Alexey A.

Larionova, Irina

LaRocque, Jan

Larsen, Anders Christian

Larue, Lionel

Lasfar, Ahmed

Lastraioli, Elena

Latham, Michael

Latif, Ayse

Latini, Francesco

Latkauskas, Tadas

Latosinsky, Steven

Latour, Sylvain

Latteri, Saverio

Lau, Andy T. Y.

Lau, Chungho

Lau, Doreen

Laura, Evangelista

Laurenzana, Anna

Laureys, Genevieve

Lauth, Matthias

Lautz, Timothy B.

Lavia, Patrizia

Lawrence, Wayne

Lawrie, Charles

Lazarević, Vladimir

Lazaris, Anthoula

Lazennec, Gwendal

Lazzarato, Loretta

Lazzeroni, Matteo

Le Bourhis, Xuefen

Le Romancer, Muriel

Le Roy, Christine

Le, Nguyen Quoc Khanh

Lea, Michael A.
Leardini, Davide

Lebaron, Richard

Lebbé, Céleste

Leber, Bettina

Leboulleux, Sophie

LeBrun, David

Lecis, Daniele

Lecka-Ambroziak, Agnieszka

Lecuru, Fabrice

Lederer, Eleanor Deland

Le-Deygen, I. M.

Lee, Alexander

Lee, Byung Chul

Lee, Chang-Hoon

Lee, Chang-min

Lee, Cheng-I

Lee, Chia-Hwa

Lee, Chien-Hsing

Lee, Dae Ho

Lee, Dakeun

Lee, Der-Yen

Lee, Ho

Lee, Hsueh-Te

Lee, Huei-Jane

Lee, Hyunju

Lee, Jae-Ho

Lee, Jaetae

Lee, Jeng-chang

Lee, Jeongmin

Lee, Jeong-yeon

Lee, Jie

Lee, Jih-Chin

Lee, Jiyoung

Lee, Joo Yong

Lee, Kang Dae

Lee, Kevin

Lee, Kin Wah Terence

Lee, Kye Young

Lee, Mei-hwa

Lee, Seung-Eun

Lee, Shao-Chen

Lee, Soo-Chin

Lee, Tsung-Hsien

Lee, Tzong-Shyuan

Lee, Wei-Hwa

Lee, Yen Chien

Lee, Yi-Yen

Lee, Yongjun

Lee, Zhenghong

Leedham, Simon

Lefebvre, Pauline M. 
Leger, David

Legler, Daniel F.

Lehen'kyi, V'yacheslav

Lehmann, Fredrik

Lehmann, Lorenz $\mathrm{H}$.

Lehner, Manfred

Lei, Wei

Leila, Khoja

Leiserowitz, Gary S.

Lejman, Monika

Lekka, Malgorzata

Lemay, Guy

Lemonnier, Loic

Leng, Roger

Lengyel, Zsuzsanna

Lenzi, Paola

Leon Mateos, Luis

Leon Serrano, Javier

León, Josefa

Leoncini, Lorenzo

Leone, Patrizia

Leong, Sai Mun

Lepage, Cecile

Lepik, Kirill V.

Leppä, Sirpa

Lerch, Michael L. F.

Lesinski, Gregory

Leslie, Kimberly

Lesniak, Wieslawa

Lessel, Davor

LeTourneau, Christophe

Leung, Dilys

Leung, Justin

Leung, Katherine

Leung, Nelson

Leupold, Jörg

Leutenegger, Marcel

Lev, Sima

Levander, Fredrik

Levi Sandri, Giovanni Battista

Levine, Herbert

Levi-Polyachenko, Nicole

Lewis-Smith, Helena

Leyva, Kathryn

Lezot, Frederic

$\mathrm{Li}$, Bo

Li, Chenglei

Li, Chi Kong

Li, Chien-Feng

Li, Chunying

Li, Fangfei
Li, Fengzhi

Li, Fuyang

Li, Guo-Min

Li, Han

Li, Hong

Li, Hui

Li, Jianfeng

Li, Jian-Jian

Li, Jiong

Li, Minglun

Li, Qin

Li, Ruei-Nian

Li, Shengwen Calvin

Li, Shizhao

Li, Taoran

Li, Weiming

Li, Weizhong

Li, Xiaohong

Li, Yamin

Li, Yang

Li, Yi-jiang

Li, Yingying

Li, Yong

Li, Yvonne

Li, Zheqi

Liagre, Bertrand

Liang, Chao

Lianos, Georgios D.

Liao, Chengheng

Liao, Guo Shiou

Liao, Peng

Liao, Yung-Feng

Liauw, Winston

Libby, Gillian

Liberini, Virginia

Libra, Massimo

Licht, Thomas

Lichtenegger, Felix

Licinia, Gouveia Zelia

Liebal, Ulf

Liersch, Torsten

Liesveld, Jane

Lifante, Jean-Christophe

Lignier, Baptiste

Likhatskaya, Galina N.

Lim, Bora

Lim, Kianhuat

Lim, Sang Moo

Lim, Tingsen Benson

Lim, Yu Jin

Limoli, Charles 
Lin, Chang-Shen

Lin, Chiao-Wen

Lin, Chien-Min

Lin, Chingyu

Lin, Chun-Shu

Lin, Eric

Lin, Huey-Jen

Lin, Jer-An

Lin, Jin-Ding

Lin, Kuo-Shyan

Lin, Liang Ting

Lin, Peter P.

Lin, Po-Han

Lin, Ruo-Kai

Lin, Shi-Ming

Lin, Shu Fu

Lin, Shu-Chun

Lin, Tzu-Ping

Lin, Wey Ran

Lin, Yan

Lin, Yen-Hung

Lin, Yu-Ching

Lin, Yueh-Chien

Linares Gómez, María

Linares, Laetitia K.

Lindenmann, Jörg

Linder, Benedikt

Lindner, Kirsten

Lindner, Lars

Lindquist, Jonathan A.

Link, Karl-Heinrich

Linnebacher, Michael

Linver, Michael

Lio, Chan-Wang Jerry

Liontos, Michalis

Liotta, Lance A.

Lipińska, Barbara

Lipiński, Piotr F. J.

Lipkowitz, Stanley

Liposits, Gábor

Lisitsyn, Nikolai A.

Lisowska, Katarzyna M.

Liss, Andrew

Lita, Adrian

Litwin, Ireneusz Bernard

Liu, Bolin

Liu, Catherine

Liu, Chi-Ming

Liu, Chung-Jung

Liu, Deli

Liu, Feng
Liu, Jun-Jen

Liu, Kebin

Liu, Kegang

Liu, $\mathrm{Li}$

Liu, Ruiwu

Liu, Shi-He

Liu, Shih-Jen

Liu, Tao

Liu, Xia

Liu, Yen-Lin

Liu, Yu-Peng

Liu, Yu-Yin

Liu, Zhi-jie (Jason)

Liu, Zhongwei

Livi, Lorenzo

Livovsky, Dan M.

Llanos Casanova, Maria

Lleonart, Matilde E.

Llop, Esther

Llop-Guevara, Alba

Llorente-Cortes, Vicenta

Llueca, Antoni

Lo Nigro, Luca

Lo Presti, Elena

Lo Sardo, Federica

Lo, Chiao

Lo, Christopher

Lo, Kwok Wai

Lo, Simon

Lobel, Lior

Lobo, Joao

Locatelli, Marzia Adelia

Löck, Steffen

Lockman, Paul R.

Loehrer, Patrick J.

Loftås, Per I.

Loginov, Dmitriy S.

Loh, Xian Jun

Lohmann, Dietmar

Lohmann, Philipp

Lohrish, Caroline

Loizidou, Maria A.

Lok, Benjamin

Lok, Hiu Chuen

Lokshin, Anna E.

Lomas, Oliver

Lombardi, Angela

Lombardi, Celestino P.

Lombardi, Giuseppe

Lombardi, Tommaso

Lonardo, Amedeo 
Lonardo, Enza

Londei, Paola

London, Nyall

Lone, Waseem

Long, Chiau Ming

Longatto-Filho, Adhemar

Longhi, Michele

Longo, Francesco

Łoniewski, Igor

Loo, Jia Min

Looijenga, Leendert H. J.

Loomans-Kropp, Holli

López, Giselle Y.

López, José I.

Lopez, Jose Javier

Lopez, Juana Serrano

Lopez-Beltran, Antonio

Lopez-Bueno, Ruben

Lopez-Camarillo, Cesar

Lopez-Charcas, Osbaldo

López-Hernández, Luz Berenice

Lopez-Jornet, Pia

Lopez-Lopez, Elixabet

Lopitz Otsoa, Fernando

Lordan, Ronan

Lorenzo, Michele

Lőrincz, Kende

Lőrinczy, Dénes

Loschi, Michael

Losco, Luigi

Loskog, Angelica

Lotti, Lavinia Vittoria

Lou, Chenguang

Louhelainen, Jari

Loveson, Katie F.

Lovšin, Nika

Lozada Nur, Francina

Lozano, Elisa

Lozano-Calderon, Santiago

Lozano-Paniagua, David

Lu, Chia-Feng

$\mathrm{Lu}, \mathrm{Chi}-\mathrm{Yu}$

$\mathrm{Lu}$, Jianfeng

Lu, Lingeng

Lu, Long-Sheng

$\mathrm{Lu}$, Tao

$\mathrm{Lu}$, Xin

Lu, Yen-Shen

Lu, Yong-Chen

Luchinat, Claudio

Luchini, Claudio
Lucia, François

Lucioni, Marco

Lucotti, Serena

Luddy, Kimberly A.

Ludovini, Vienna

Ludvigsen, Maja

Ludvikova, Marie

Ludwig, Heinz

Ludwig, Michael

Lueong, Smiths S.

Lukas, Rimas V.

Lulla, Amriti

Lulli, Valentina

Luminari, Stefano

Lun, Xiaokang

Lundgren, Catharina Ihre

Lundqvist, Andreas

Lunov, Oleg

Luo, Danmeng

Luo, Qun

Lupiáñez, José A.

Lupu, Mihai

Lurje, Isabella

Luscinskas, Francis William (Bill)

Lustig, Arthur

Łuszczki, Jarogniew

Luxton, G. W. Gant

Luyt, Len

Lyng, Fiona

Lynge, Elsebeth

Lyros, Orestis

Lyu, Hui

Lyu, Ruitu

M'kacher, Radhia

Ma, Yafeng

Maccari, Alberto

Maccio, Antonio

Mace, Peter

Macek Jílková, Zuzana

Macerola, Elisabetta

Machairas, Nikolaos

Machens, Andreas

Macias, Rocio I. Rodriguez

Maciej, Ciesla

Maciejewski, Ryszard

Mack, Elisabeth Karin Maria

MacLeod, Nicholas

Maçôas, Ermelinda

Macurek, Libor

Madamsetty, Vijay Sagar

Maddeboina, Krishnaiah 
Mader, Robert

Mader, Sylvie

Madhankumar, Achuthamangalam B.

Madhukar, Burra

Madka, Venkateshwar

Madkour, Aicha

Maeda, Kiyoshi

Maes, Evelyne

Maestro, Roberta

Maffini, Enrico

Magagnoli, Federica

Maggi, Enrico

Maggi, Leonard B.

Maggialetti, Nicola

Maggiolini, Marcello

Magherini, Francesca

Magkouta, Sophia

Maglietta, Giuseppe

Magnaldo, Thierry

Magnani, Corrado

Magnes, Teresa

Magnoni, Francesca

Magrini, Stefano Maria

Mahadevan, Daruka

Mahesh, Kasthuri

Mahmood, Niaz

Mahran, Amr

Maia, Cláudio

Maida, Ivana

Maida, Pietro

Maina, Flavio

Maiorano, Domenico

Mais, Valerio

Maisano, Domenico

Maita, Hiroshi

Majchrzak-Celińska, Aleksandra

Majem, Blanca

Majumder, Samarpan

Majumder, Sarmila

Mak, Nai-Ki

Makena, Monish Ram

Makimoto, Atsushi

Makishima, Hirokazu

Makuch-Kocka, Anna

Makvandi, Pooyan

Malaguarnera, Michele

Malapelle, Umberto

Malara, Natalia

Malavasi, Fabio

Malek, Anastasia

Malek, Kamilla
Maleki, Zahra

Malgor, Ramiro

Małkiewicz, Bartosz

Mallamaci, Rosanna

Mallio, Carlo Augusto

Malpeli, Giorgio

Malvezzi, Matteo

Mamessier, Emilie

Mamidi, Murali

Mamouni, Kenza

Man, Francis

Manapov, Farkhad

Mancarella, Caterina

Mancini, Francesca

Mancini, Maicol

Mancini, Mariangela

Manconi, Andrea

Mancuso, Salvatrice

Manda, Gina

Manem, Venkata

Manes, Santos

Manet, Evelyne

Manfredi, Sylvain

Mangieri, Domenica

Mangla, Ankit

Mango, Lucio

Mangone, Lucia

Mani, Sridhar

Manirujjaman, M.

Mann, Karen M.

Manneh, Ray

Mannelli, Francesco

Manning, Amity L.

Mannini, Antonella

Manolopoulos, Spyros

Manousaki, Despoina

Manova, Vasilissa

Mansi, Rosalba

Mansier, Olivier

Mantini, Giovanna

Mantovani, Fiamma

Manzo-Merino, Joaquin

Manzotti, Gloria

Mapelli, Paola

Mapuskar, Kranti A.

Marampon, Francesco

Marasco, Giovanni

Marc, Janja

Marçais, Antoine

Marcato, Paola

Marchal, Frédéric 
Marchand, Tony

Marche, Patrice N.

Marchesi, Federica

Marchetti, Luca

Marchetti, Marco

Marchica, Valentina

Marchini, Cristina

Marco, Ardigo

Marcondes Lerario, Antonio

Marconi, Alessandra

Marconi, Giovanni

Marcos Gragera, Rafael

Marcu, Loredana

Marculescu, Rodrig

Marcus, Hani J.

Margenthaler, Julie A.

Marí Alexandre, Josep

Maria Carmela, Bonaccorsi Di Patti

María Huerta, José

Marian, Brigitte

Mariani-Costantini, Renato

Marignani, Paola

Marignol, Laure

Marimuthu, Parthiban

Marin, Oskar

Marinas-Pardo, Luis Antonio

Marini, Cecilia

Marini, Herbert Ryan

Marinko, Tanja

Marino, Franca

Marino, Maria

Mario, Bignardi

Mariotti, Francesca Romana

Mariotti, Stefano

Marjanski, Tomasz

Mark, Tomer M.

Markiewicz, Aleksandra

Markiewicz, Anna

Märkl, Bruno

Markopoulos, Georgios S.

Markova, Svetlana

Marko-Vargä, György

Markowska, Anna

Marks, Daniel L.

Marks, Lianna

Marmorino, Federica

Marodon, Gilles

Marongiu, Francesco

Marotz, Jörg

Marques, M. Matilde

Marquez, Joana
Marra, Fabio

Marra, Paolo

Marsh, Deborah J.

Marshall, Lindsay

Martell, Kevin

Martelli, Alberto Maria

Martelli, Maria Paola

Marti, Rosa M.

Marti-Garcia, Celia

Martin, Alejandro

Martin, Daniel

Martin, Elizabeth M.

Martin, Michèle

Martin, Robert C. G.

Martin, Simon S.

Martin, Tracey

Martina, Emanuela

Martínez Beamonte, Roberto

Martínez López, Joaquín

Martinez Macias, Maria Isabel

Martinez Usatorre, Amaia

Martínez, Constantino

Martínez, Lidia

Martinez, Magaly

Martinez-Fernandez, Lara

Martínez-Galiano, Juan Miguel

Martínez-López, Joaquín

Martínez-Pérez, Carlos

Martínez-Soler, Fina

Martinez-Tapia, Claudia

Martinez-Useros, Javier

Martinez-Zubiaurre, Inigo

Martin-Hirsch, Pierre

Martinho, Olga

Martini, Andrea

Martini, Maurizio

Martini, Veronica

Martins, José A.

Martins, Natália

Martín-Sánchez, Esperanza

Martins-Neves, Sara R.

Martín-Vasallo, Pablo

Martynowicz, Helena

Maruoka, Yasuhiro

Marusyk, Andriy

Maruyama, Tessho

Marvaso, Giulia

Marverti, Gaetano

Marx, Alexander

Mary, Didier

Masamune, Atsushi 
Mascagni, Domenico

Mascarella, Marco

Mascheroni, Pietro

Maschmeyer, Georg

Mascitti, Marco

Mascolo, Massimo

Masedu, Francesco

Masel, Eva Katharina

Maslowski, Kendle

Mason, Ralph

Mason, Warren P.

Masqué-Soler, Neus

Massari, Francesco

Massarut, Samuele

Massimino, Maura

Massone, Cesare

Massotte, Dominique

Massoumi, Ramin

Masszi, Andras

Mastrangeli, Massimo

Mastrangelo, Stefano

Mastronuzzi, Angela

Masuda, Kiyoshi

Masuda, Mari

Masuda, Norikazu

Masuda, Takaaki

Masuda, Takeshi

Masui, Kenta

Masuzaki, Ryota

Matei, Irina

Mateos, Pilar Fernández

Materka, Andrzej

Matesic, Lidia

Matet, Alexandre

Matheu, Ander

Mathew, Stephen

Mathieu, David

Mathieu, Marie-Christine

Mathieu, Véronique

Matiatou, Maria

Mato, Eugenia

Matozaki, Takashi

Matrisian, Lynn M.

Matsubara, Saburo

Matsuda, Yoko

Matsui, Yoshiyuki

Matsuki, Takahiro

Matsukuma, Satoshi

Matsumoto, Kazumasa

Matsumoto, Naoki

Matsumoto, Seiichi
Matsumoto, Yoshihisa

Matsuo, Yukiko

Matsusaki, Takashi

Matsuzaki, Shinya

Matthies, Cordula

Matthiesen, Rune

Matthiessen, Peter

Mattonen, Sarah

Matulic, Maja

Matveeva, Olga

Matyjaszek-Matuszek, Beata

Maugeri, Andrea Giuseppe

Maugeri, Rosario

Maughan, Benjamin L.

Maura, Calvani

Maurea, Simone

Maurer, Jochen

Maurer, Stefanie

Mauri, Davide

Maurizio, Aurora

Maury, Jean Michel

Mausolf, Edward J.

Mavinkurve-Groothuis, Annelies M. C.

Mavrogonatou, Eleni

Mavroidi, Barbara

Mavros, Michail

Mavroudis, Dimitrios

Mavuluri, Jayadev

Mawhinney, Thomas

Maximov, Ivan

May, Felicity

Mayani, Hector

Maycotte, Paola

Mayer, Arnulf

Maynard, Janielle P.

Mayo, Samantha J

Mayoh, Chelsea

Mazan-Mamczarz, Krystyna

Mazurek, Agnieszka M.

Mazurek, Urszula

Mazzanti, Chiara Maria

Mazzei, Maria Antonietta

Mazzola, Michele

Mazzoni, Maria

Mazzucchelli, Roberta

McArdle, Stephanie

McCaughan, Geoff

McCaughan, Geoffrey William

McCaul, James Anthony

McClurg, Urszula

McComb, Scott 
McCullagh, James

Mcdonald, Jeffrey

McEwen, Gayle

McGowan, Eileen

McGrowder, Donovan

McIntosh, Stuart A.

McKeon, Frank

McKinstry, Karl Kai

McLean, Karen

McMullin, Mary Frances

McNally, Lacey R.

McNally, Orla

McReynolds, Lisa J.

Meade, Aidan D.

Mèchinaud, Françoise M.

Medenwald, Daniel

Medipally, Dinesh

Meehan, Katie

Mego, Michal

Mehrabi, Arianeb

Mehta, Ambereen

Mehta, Gaurav A.

Mehta, Sunali

Mehterov, Nikolay

Mei, Kuo-Ching

Mei, Ya-Fang

Meiburger, Kristen M.

Meignan, Michel

Meiler, Johannes

Meiser, Johannes

Melcón, Gallego

Melendy, Thomas

Melenhorst, Jan Joseph

Melgaço, Juliana G.

Meli, Francesco

Mellai, Marta

Mellitzer, Georg

Melloni, Elisabetta

Melo, Sonia A.

Melstrom, Kurt A.

Meltzer, Sebastian

Mena, Marisa

Menard, Armelle

Mendichovszky, Iosif

Menè, Paolo

Menéndez-Menéndez, Javier

Meng, Xiangbing

Menghi, Francesca

Menichetti, Luca

Menke, Andre

Mennerich, Daniela
Menon, Dinoop Ravindran

Mensink, Hanneke W.

Mercer, Claire

Mercke, Claes

Merkel, Susanne

Merli, Pietro

Merola, Elettra

Merolla, Francesco

Mertens, Christina

Merz, Valeria

Mesguich, Charles

Messaritakis, Ippokratis

Messersmith, Amy

Messiha, Hadi

Messner, Franka

Mesuraca, Maria

Metere, Alessio

Metovic, Jasna

Metzen, Eric

Metzler, Markus

Meyer Zu Heringdorf, Dagmar

Meyer, Hans-Jonas

Meyer, Moritz

Meyer, Peter

Meyers, Craig

Mezencev, Roman

Mezi, Silvia

Mezõsi, Emese

Miah, Aisha

Miao, Lingling

Miceli, Vitale

Michael, Agnieszka

Michael, Michael

Michaelis, Martin

Michalis, Eurydiki

Michalsen, Andreas

Michaud, Karine

Michelacci, Yara M.

Michelhaugh, Sharon K.

Michelle, Palmieri

Michels, Judith

Michikawa, Takehiro

Micke, Patrick

Miederer, Matthias

Migliaccio, Antimo

Mihara, Keichiro

Mihaylov, Plamen

Mikaelyan, Arsen S.

Miklikova, Svetlana

Miklós, Gyöngy

Mikolajczak, Renata 
Mikolajczyk, Rafael

Mikropoulos, Christos

Mikula, Michal

Milanowski, Maciej

Milczarek, Małgorzata

Milella, Michele

Mileo, Anna Maria Nive

Miles, Lindsey

Miletic, Hrvoje

Miliauskas, Skaidrius

Milic, Marina

Miligi, Lucia

Militello, Carmelo

Miljković, Miloš D.

Miller, Anthony B.

Miller, Ian Steuart

Miller, John H.

Miller, Kimberly

Miller, Vandana

Miller, William B.

Miller, Wilson $\mathrm{H}$.

Millesi, Matthias

Mills, Ken

Min, Irene

Mina, Roberto

Minafra, Luigi

Minami, Yasunori

Minami, Yosuke

Minamikawa, Takeo

Minamimoto, Ryogo

Minea, Radu

Minervini, Giovanni

Minna, Emanuela

Minnelli, Cristina

Minucci, Angelo

Minutolo, Filippo

Mio, Catia

Mirabilii, Simone

Miricescu, Daniela

Mirjolet-Didelot, Céline

Mirlekar, Bhalchandra

Mirzaei, Siroos

Mirzayans, Razmik

Mischkulnig, Mario

Miserocchi, Giacomo

Mishra, Avanish

Mishra, Manoj

Mishra, Rosalin

Mishra, Sandeep Kumar

Miskiewicz, Piotr

Miszczyk, Justyna
Mita, Koji

Mitani, Seiichiro

Mitchell, Paul

Mitjavila, Mercedes Casanova

Mitobe, Yuichi

Mitola, Stefania

Mitra, Mithun

Mitra, Ranjana

Mitra, Sumegha

Mitsogiannis, Iraklis

Mitsuishi, Yoichiro

Miura, Kouhei

Miura, Sayaka

Miyagi, Yohei

Miyamoto, Hirotaka

Miyamoto, Ikuya

Miyanaga, Akihiko

Miyazaki, Masaru

Miyazawa, Masaaki

Miziak, Barbara

Mizukami, Tatsuji

Mizuno, Shugo

Mo, Fei

Mo, Lein-Ray

Mobasheri, Ali

Mochizuki, Satsuki

Moderau, Nina

Moertl, Simone

Mogler, Carolin

Mograbi, Baharia

Mohammed, Altaf

Mohan, Meera

Mohanty, Vakul

Mohapatra, Bhopal

Mohibi, Shakur

Mohl, Jonathon Edward

Möhlendick, Birte

Mohr, Thomas

Moia, Riccardo

Mojarad, Ehsan Nazemalhosseini

Mojsilović, Slavko B.

Mojumdar, Kamalika

Mokbel, Kefah

Moldovanu, Simona

Moles, Anna

Moles, Jean-pierre

Molfetta, Rosa

Molica, Matteo

Molin, Daniel

Molina, Eric Suero

Molina, Thierry Jo 
Molina-Cerrillo, Javier

Moll, Annette

Möller, Gabriele

Momčilović, Stefan D.

Monacelli, Fiammetta

Monaco, Giovanni

Moncayo, Roy

Mondal, Goutam

Mondal, Tanmoy

Mondelli, Mario U.

Monsalve, Maria

Montalban-Arques, Ana

Montalbano, Mauro

Montano, Nicola

Monteleone, Giovanni

Montemurro, Nicola

Montorsi, Marco

Montrucchio, Giuseppe

Monzani, Fabio

Moon, Dominic

Moon, Ejung

Moon, Sung-Kwon

Moore-Carrasco, Rodrigo

Moormann, Ann

Moosa, Mahdi Muhammad

Mora, Francesco

Mora, Jaume

Morais, Mauricio

Morais, Rui

Morak-Młodawska, Beata

Morales, Humberto

Morales, Jose Manuel

Morales, Julio

Morales, Serafin

Mora-López, Laura

Morandi, Luca

Morani, Federica

Moreaux, Jerome

Morel, Alain

Morel, Pierre

Morelli, Luca

Morelli, Maria Beatrice

Morello, Silvana

Moreno, Carlos S.

Moreno, Estefanía

Moreno, J. J.

Moret, Francesca

Moretti, Sonia

Morgan, Ethan L.

Morgan, Michael

Morganti, Alessio Giuseppe
Mori, Mattia

Mori, Yasuhisa

Morigi, Rita

Moris, Lisa

Morita, Yoshihiro

Morito, Kurata

Moritz, Rose K. C.

Morizane, Ryuji

Morjani, Hamid

Morkel, Markus

Moro, Francesca

Morotti, Alessandro

Morra, Francesco

Morra, Simone

Morrione, Andrea

Morris, Don

Morrison, Jo

Morrissey, Maria

Morselli, Simone

Moscetti, Luca

Moser, Bernhard

Mosqueira, Vanessa Carla Furtado

Moss, Esther Louise

Motamed, Cyrus

Motea, Edward A.

Motegi, Atsushi

Motiam, Ahmed El

Motoi, Fuyuhiko

Motola, Domenico

Motoyama, Satoru

Moulik, Sabyasachi

Moulin, Alexandre

Mouneimne, Ghassan

Mountzios, Giannis

Moura, David S.

Mourtada-Maarabouni, Mirna

Mowday, Alexandra

Mpakali, Anastasia

Mravec, Boris

Muallem, Mustafa Zelal

Mudryj, Maria

Mueller, Mathias

Muenst, Simone E.

Muether, Michael

Muggeo, Paola

Muhammad, Jibran Sualeh

Mühlebner, Angelika

Mukherjee, Sudip

Mukhopadhyay, Suman

Mulhearn, Ben

Mulholland, Eoghan 
Müller, Klaus Gottlob

Muller, Laurent

Muller, Mandy

Müller, Stefan

Müller-Decker, Karin

Mun, Seong K.

Munakata, Satoru

Munir, Khushboo

Muniraj, Nethaji

Munker, Reinhold

Munkley, Jennifer

Muñoz Sáez, Miguel

Munz, Christian

Muragaki, Yoshihiro

Murata, Soichiro

Murata, Takayuki

Muresan, Mihai Stefan

Murotani, Kenta

Murphy, Andrew

Murphy, Brittany

Murphy, Brona

Murray, David

Murray, Jayne

Murray, Paul

Murthy, Divya

Murugan, Sengottuvelan

Muruganandan, Sanjeevan

Murugesan, Kanagavel

Murugesan, Selvasankar

Muscarella, Lucia Anna

Muscolini, Michela

Musi, Gennaro

Mussolin, Lara

Muz, Barbara

Muzio, Marta

Muzza, Marina

Mycielska, Maria

Mymryk, Joe

$\mathrm{Na}$, Kiyong

Nabiee, Romina

Nadanaka, Satomi

Nadeau, Jay L.

Nagano, Hiroaki

Nagasaka, Kazunori

Nagata, Yosuke

Nagelkerke, Anika

Nagler, Arnon

Nagrani, Rajini

Nahain, Abdullah

Naidu Chitrala, Kumaraswamy

Naillat, Florence
Nair, Praful R.

Naito, Tateaki

Naito, Yutaka

Najahi Missaoui, Wided

Nakagawa, Hidewaki

Nakagawa, Takuro

Nakahara, Tomomi

Nakai, Yousuke

Nakajima, Osaniu

Nakajima, Takahiro

Nakajima, Takahito

Nakamura, Naoya

Nakamura, Takuro

Nakamura, Yasuhiro

Nakamura, Yoshiyuki

Nakamura, Yusuke

Nakanishi, Naohiko

Nakano, Kenji

Nakano, Masahito

Nakano, Toshiaki

Nakashima, Souichi

Nakayama, Noriyuki

Nakayama, Robert

Nakazawa, Tsutomu

Nallanthighal, Sameera

Nalvarte, Ivan

Nami, Babak

Namløs, Heidi M.

Nanda, Neha

Nanduri, Siva Lahiri Kanth

Nannini, Margherita

Narayana, Shalini

Nardi, Mariateresa

Narita, Norihiko

Narkhede, Mayur

Naryzhny, Stanislav N

Nascimento, Bruno F. O.

Naselli, Angelo

Nash, Robert

Nashine, Sonali

Nasillo, Vincenzo

Nason, Gregory

Nass, Norbert

Nassir, Fatiha

Nassiri, Mehdi

Natalello, Antonino

Natarajan, Sivaraman

Nath, Shubhankar

Natkunam, Yasodha

Natsumeda, Manabu

Naumann, Nicole 
Nausica, Montalto

Nava, Victor

Navarria, Pierina

Navarro, José-Tomás

Navran, Arash

Nawas, Afshan Fathima

Nawaz, Mohd Imtiaz

Nawroth, Roman

Nayak, Jasmir G.

Nayar, Manu

Nazaruk, Ewa

Nazio, Francesca

Neagoe, Ioana Berindan

Neagoe, Octavian

Nechaev, Sergei

Neels, Oliver

Nees, Matthias

Negoro, Hiromitsu

Negri, Gian Luca

Negri, Rodolfo

Neill, Stewart G.

Nel, Ivonne

Nelson, Grady

Nemunaitis, John J.

Neophytou, Christiana M.

Nephew, Kenneth P

Nerreter, Thomas

Nervi, Clara

Nessim, Carolyn

Neufert, Clemens

Neuhaus, Jochen

Neureiter, Daniel

Neuwelt, Alexander J.

Neven, Patrick

Nevi, Lorenzo

Nevzorova, Yulia

Newhouse, Ian

Ney, Douglas E.

Ngezahayo, Anaclet

Ngo, Thuy

Nguyen, Frédérique

Nguyen, Jane K.

Nguyen, Minhhuyen T.

$\mathrm{Ni}$, Wei

Niazi, Khalid

Nibali, Marco Conti

Nicolas, Emmanuelle

Nicoletti, Ferdinando

Nicos, Marcin

Nicoś, Marcin

Niculae, Dana
Nie, Daotai

Nieland, John Dirk Vestergaard

Nielsen, Finn Cilius

Nielsen, Kari

Nieminen, Taina $\mathrm{T}$.

Niemiro, Grace M.

Nienhüser, Henrik

Niessner, Heike

Niewald, Marcus

Nii, Teruki

Niikura, Ryota

Nijhof, Inger

Nijland, Marcel

Nijnatten, Thiemo

Nikas, Ilias P.

Nikfar, Shekoufeh

Nikitovic, Dragana

Nikolova, Biliana

Nimura, Keisuke

Ning, Shunbin

Nirala, Niraj K.

Niscola, Pasquale

Nishida, Haruto

Nishida, Hiroko

Nishida, Shozo

Nishida, Toshiro

Nishida, Tsutomu

Nishikawa, Hiroki

Nishimoto, Koshiro

Nishimura, Noriyuki

Nishina, Hiroshi

Nishio, Mizuho

Nishio, Shin

Nishizawa, Toshihiro

Nissinen, Tuuli A.

Nisticò, Steven Paul

Nitika, Nitika

Nitulescu, George Mihai

Nitulescu, Mihai

Nixon, Daniel W.

Niza, Enrique

Njoku, Kelechi

Njor, Sisse Helle

Noberini, Roberta

Nobili, Stefania

Nobis, Max

Nogova, Lucia

Noguera, Nélida Inés

Noh, Jae Myoung

Noh, Ji-Yoon

Noh, Woo Chul 
Nonnekens, Julie

Noonan, Douglas

Noorani, Imran

Noordermeer, Sylvie

Noothalapati, Hemanth

Norcic, Gregor

Nordström, Anders

Nordstrom, Robert

Noro, Rintaro

Nøst, Therese Haugdahl

Noubissi-Kamdem, Felicite

Novakova, Zora

Novick, Daniela

Nowak, Daniel

Nowak, Michal S.

Nowakowska-Zajdel, Ewa

Ntanasis-Stathopoulos, Ioannis

Nuciforo, Paolo

Nuhn, Philipp

Numakura, Kazuyuki

Nürnberg, Sylvia

Ny, Lars

Nylander, Karin

Nylen, Carolina

Nyström, Alexander

O'Dwyer, Michael

O'Leary, John

O'Reilly, Seamus

O'Rourke, Colm

O'Toole, Sharon A.

Oancea, S. Cristina

Obata, Yuuki

Oberacker, Eva

Obermayr, Eva

Obi, Shuntaro

Obinata, Daisuke

Ochieng, Josiah

Ocker, Matthias

Oda, Tsukasa

Oddone, Enrico

Oechsle, Karin

Oehler, Rudolf

Oehme, Ina

Oerlemans, Simone

Offer, Steven

Offermann, Anne

Öfner-Velano, Dietmar

Ogawa, Tetsuya

Ogino, Shuji

Ogino, Takashi

Ogiwara, Hideaki
Ognibene, Marzia

Ogris, Manfred

Oh, Eun-Taex

Oh, Seh-Hoon

Oh, Sekyung

Ohara, Toshiaki

Ohashi, Manabu

Ohba, Kojiro

Ohishi, Tomokazu

Ohkawa, Kazuyoshi

Ohkuri, Takayuki

Ohno, Hitoshi

Ohnuki, Hidetaka

Ohtsuka, Takashi

Ohyama, Tetsuji

Oikawa, Kosuke

Oji, Yusuke

Okabe, Seiichi

Okada, Tomoyo

Okami, Kenji

Okamoto, Mariko

Oki, Kenji

Okita, Yoshiko

Oklu, Rahmi

Okonechnikov, Konstantin

Okonogi, Noriyuki

Okosun, Jessica

Oksenych, Valentyn

Okubo, Hironao

Okumura, Toshiyuki

Okumura, Yasuhiro

Okuno, Scott

Okusaka, Takuji

Olaru, Octavian Tudorel

Olaya Abril, Alfonso

Olbryt, Magdalena

Ole Schmidt, Nils

Olin, Michael

Oliveira, Maria Cristina

Oliveira, Sonia M.

Oliver, Lisa

Ollauri-Ibáñez, Claudia

Olmo, Alberto

Olsen, Maja

Olsson, Lars E.

Omerovic, Jasminka

Omilusik, Kyla D.

Onadim, Zerrin

Ono, Yoshihiro

Onoda, Naoyoshi

Opalińska, Marta 
Opländer, Christian

Oprea - Lager, Daniela

Opyrchal, Mateusz

Oratz, Ruth

Orchard, Sandra

Örd, Tiit

Örd, Tönis A.

Ordonez-Moran, Paloma

Orefice, Nicola

Orimo, Akira

Orion, Itzhak

Orlandella, Francesca Maria

Orlandi, Rosaria

Orlova, Anna

Orme, Jacob

Orsi, Nic

Ortiz, Raul

Ortiz-Cuaran, Sandra

Osaki, Mitsuhiko

Oshima, Tadayuki

Osti, Mattia Falchetto

Ostrowski, Marcin

Ostróżka-Cieślik, Aneta

Oswald, Franz

Ota, Atsuhiko

Ota, Takayo

Othman, Ahmed

Otowa, Yasunori

Otsuka, Isao

Ott, German

Ottaiano, Alessandro

Ottaviano, Margaret

Otterbein, Leo

Ou, Da-Liang

Ou, Fangshu

Ouaissi, Mehdi

Oudard, Stephane

Ouerfelli, Ouathek

Ouled-Haddou, Hakim

Oulhen, Marianne

Oura, Kyoko

Ouseph, Madhu

Ouzounis, Christos

Ove, Roger

Owczarczyk, Katarzyna

Owen, Carolyn

Owen, Gareth

Owen, Katie

Owens, Philip

Oyoshi, Takanori

Ozaki, Shuji
Ozeki, Yasuhiro

Ozretić, Petar

Ozsvari, Bela

Ozuner, Gokhan

Paakinaho, Ville

Paauwe, Madelon

Pabst, Andreas

Pabst, Thomas

Pacak, Karel

Pach, Radoslaw

Paciotti, Marco

Packiam, Vignesh T.

Padi, Sathish

Padler-Karavani, Vered

Padmanabhan, Achuth

Padmanabhan, Jaya

Pagano, Allan F.

Pagano, Nico

Pagella, Fabio

Pagenstecher, Axel

Pagliara, Monica Maria

Pagliuca, Simona

Pagotto, Sara

Paholcsek, Melinda

Pahwa, Roma

Paíno, Teresa

Painschab, Matthew

Paireder, Matthias

Pal, Ajay

Pal, Krishnendu

Pala, Andrej

Palacios Calvo, Jose

Palacios García, Daniela

Palaia, Gaspare

Palamà, Ilaria Elena Lena

Palazzari, Elisa

Palazzo, Elisabetta

Palazzo, Luca

Paleari, Laura

Paleologu, Constantin

Palladini, Arianna

Pallagi, Petra

Pallavi, Manral

Pallavicini, Gianmarco

Palles, Claire

Palleschi, Alessandro

Palmer, Gregory M.

Palmieri, Giuseppe

Palmieri, Lola Jade

Palmieri, Maria

Palmqvist, Richard 
Palomo, Laura

Palumbo, Carlotta

Palumbo, Giuseppe A.

Paluszczak, Jaroslaw

Pampena, Riccardo

Pan, Chun-Hsu

Pan, Edward

Pan, Jiayi

Pan, Wen-Yu

Panaro, Fabrizio

Panda, Amaresh

Pandey, Amit

Pandey, Gaurav

Pandey, Rajeev

Pandey, Ritu

Pangault, Céline

Panigrahi, Gatikrushna

Panneerdoss, Subbarayalu

Pantalone, Mattia Russel

Pantel, Klaus

Pantelyushin, Stanislav

Panzuto, Francesco

Paolillo, Carmela

Paolino, Giovanni

Paoluzzi, Luca

Paone, Gaetano

Papaccio, Federica

Papachristodoulou, Alexandros

Papachristos, Alexander J.

Papadaki, Chara

Papadaki, Maria

Papadakos, Konstantinos

Papadimitriou, Christos

Papadodima, Olga

Papadopoulos, Othon

Papamatheakis, Joseph (Sifis)

Papassotiriou, Iannis

Papoutsoglou, Panagiotis

Pappa, Aglaia

Paprocka, Justyna

Paprottka, Philipp M.

Paradela, Sabela

Paranjape, Anurag

Paraskeva, Efrosyni

Parat, Marie-Odile

Pardal-Refoyo, José Luis

Pardo-Pérez, Maria

Parekh, Chintan

Parente, Paola

Paret, Claudia

Parida, Sheetal
Paris, Francois

Park, Boyoung

Park, Henry S.

Park, Hyun Woo

Park, Hyung Seok

Park, Hyunjin

Park, In Ja

Park, Jee Soo

Park, Jeong A.

Park, Jong Kook

Park, Ki-cheong

Park, Kyung Chan

Park, Kyung Sun

Park, Mi Kyung

Park, Sue K.

Park, Thomas In-Hyeup

Park, Wooram

Parker, Seth

Parkinson, E. Kenneth

Parmigiani, Elena

Parolini, Isabella

Paronetto, Maria Paola

Parra-Membríves, Pablo

Parrinello, Giampiero

Parrozzani, Raffaele

Parsons, Jason

Partanen, Marita

Parys, Maciej

Pascale, Rosa

Pascali, Giancarlo

Pascual, Gloria

Pasek, Małgorzata

Pasetto, Anna

Pasmant, Eric

Pasqualetti, Giuseppe

Pasquali, Claudio

Pasquier, Eddy

Passeri, Benedetta

Pastorek, Jaromir

Pastoret, Cédric

Pastorino, Lorenza

Pastré, David

Pata, Giacomo

Patankar, Manish

Patel, Girijesh Kumar

Patel, Nibedita

Patelarou, Athina

Pateras, Ioannis S.

Patócs, Attila

Patrone, Mauro

Patrono, Damiano 
Patsoukis, Nikolaos E.

Patterson, Pandora

Pattnaik, Bikash

Paudel, Bishal

Paudel, Keshav Raj

Paul, Dennis

Paul, Manash K.

Paulides, Maarten

Paulo, António

Paulo, Paula

Pavlik, Edward

Pavlik, Edward J.

Pavlin, Mojca

Pavlova, Elena

Pavlova, Galina

Pavone, Mary Ellen G.

Paweł, Kaliński

Pawelczyk, Tadeusz

Payabvash, Seyedmehdi Mehdi D.

Payne, Annette

Pazgan-Simon, Monika

Pearl, Michael L.

Pecorari, Giancarlo

Pecsi, Daniel

Pedrosa, Pedro

Pei, Yonggang

Peiris, Casey

Peitl, Petra

Peitzsch, Claudia

Peixoto, Andreia

Pejchal, Jaroslav

Pęksa, Rafał

Pellegrini, Cristina

Pellegrini, Manuela

Pellegrino, Marsha

Pellegrino, Paolo

Pellegrino, Rossella

Pellerino, Alessia

Pelletier, Joffrey

Pellicano, Rinaldo

Peltomäki, Päivi

Pemov, Alexander

Peña-Chilet, Maria

Peña-Chilet, María

Peña-Llopis, Samuel

Penalva, Luiz

Pende, Daniela

Peng, Dunfa

Peng, I-Chen

Penninckx, Sébastien

Pennock, Nathan
Pentimalli, Francesca

Peperzak, Victor

Pera, Alejandra

Peralto, Jose Luis Rodriguez

Peran, Ivana

Perego, Michela

Pereira Lemos, Julia

Pereira, David

Pereira, Patrícia A.

Pereira, Sofia S.

Pereira-Veiga, Thais

Pérès, Elodie A.

Peretti, Sara

Pérez Romasanta, Luis Alberto

Perez, Kimberly

Pérez-García, José Manuel

Perez-Hernandez, Concepción

Perez-Ruiz, Elizabeth

Pérez-Santos, Martín

Perez-Stable, Carlos

Pergolizzi, Denise

Perini, Giordano

Peris, Ketty

Perła-Kaján, Joanna

Perna, Giuseppe

Pérol, David

Peron, Julien

Peroni, Edoardo

Perra, Teresa

Perrakis, Aristotelis

Perrone, Anna Myriam

Perrone, Francesco

Persano, Stefano

Persichetti, Paolo

Persico, Marcello

Persson, Emma

Persson, Jenny

Peters, Christian

Petersen, Ole William

Pethő, Zoltán

Petitprez, Florent

Petrella, Francesco

Petrik, Jim

Petrone, Maria Chiara

Petroni, Marialaura

Petros, John A.

Petrov, Anton

Petrova, Boryana

Petruczynik, Anna

Petry, Andreas

Pettinato, Giuseppe 
Pettit, Kristen

Peulen, Olivier

Peyrl, Andreas

Pezone, Antonio

Pezzilli, Raffaele

Pezzolo, Annalisa

Pfahlberg, Annette

Pfahlberg, Annette B.

Pfister, Astrid S.

Phatarpekar, Prasad

Phelan, James J.

Phélip, Jean Marc

Philipp, Manfred

Phillips, Kelly A.

Piana, Michele

Picardo, Sarah L.

Piccardo, Arnoldo

Piccin, Andrea

Piccinin, Elena

Picco, Vincent

Picimbon, Jean-François

Piechota-Polanczyk, Aleksandra

Pierelli, Luca

Pierfrancesco Francesco, Bassi

Pieroni, Luisa

Pietra, Gabriella

Pietrangelo, Laura

Pietrusiński, Michal

Pietruszewska, Wioletta

Pigazzi, Martina

Pignot, Géraldine

Pijnenborg, Hanny

Pilarsky, Christian

Pilatus, Ulrich

Pileri, Stefano

Pilleron, Sophie

Pillozzi, Serena

Pilmane, Māra

Pina, Annick

Pina, Cristina

Pincus, Seth

Pindyurin, Alexey V.

Pineda, Silvia

Piñeiro, Roberto

Pink, Daniel

Pinkawa, Michael

Pintea, Bogdan

Pinto, Carmine

Pinto, Eleonora

Pinto, Filipe

Pinto, Mafalda
Pinzani, Pamela

Pioli, Claudio

Piotrowska, Ewa

Piovesan, Alessandro

Piperi, Christina

Piperno-Neumann, Sophie

Pipkorn, Patrik

Piri, Reza

Pirich, Christian

Piris Pinilla, Miguel Ángel

Pirtea, Laurentiu

Pisani, Carla

Pisani, Laura Francesca

Piva, Roberto

Piva, Terrence

Pizzi, Marco

Placer, Carlos

Planque, Chris

Plantinga, Maud

Platta, Harald

Plattner, Rina

Plazzer, John Paul

Plesa, Adriana

Pluck, Graham

Pluquet, Olivier

Plymate, Stephen R.

Pocard, Marc

Podar, Klaus

Poder, Thomas G.

Podhorska-Okołów, Marzena

Podlipnik, Sebastian

Poggio, Francesca

Pohlan, Julian

Poirier, Donald

Poirier, Guy G.

Poirot, Marc

Polakowski, Nicholas J.

Polańczyk, Andrzej

Polano, Maurizio

Polap, Dawid

Polese, Lino

Polesel, Jerry

Polesello, Cédric

Polgarova, Kamila

Poli, Valeria

Polici, Michela

Polimeno, Lorenzo

Poliseno, Laura

Polishchuk, Roman

Pollice, Alessandra

Pollok, Karen E. 
Polz-Dacewicz, Malgorzata

Pompano, Rebecca

Pond, Amber L.

Pond, Gregory

Pongracz, Judit E.

Ponik, Suzanne

Ponikwicka-Tyszko, Donata

Pons, Sebastian

Ponz-Sarvise, Mariano

Pop, Laurentiu

Popanda, Odilia

Poplawski, Piotr

Popli, Pooja

Popovic, Snezana

Popper, Helmut H.

Poptani, Harish

Porcu, Eleonora

Porcu, Luca

Porpodis, Konstantinos

Portella, Giuseppe

Portman, Neil

Porto, Beatriz

Poschke, Isabel

Poškus, Eligijus

Possamai, Lucia A.

Posthaus, Horst

Potempa, Lawrence A.

Potez, Marine

Potočnik, Nejka

Pouget, Jean-Pierre

Pouliakis, Abraham

Pouliquen, Daniel Loïc

Pourbaghi, Milad

Pourkarim, Mahmoud Reza

Poxleitner, Philipp J.

Poyet, Jean-Luc

Pozzi, Nicola

Prabhu, Antony Herold

Prabhu, Lakshmi

Prada, Ilaria

Pranckevičienè, Aistė

Prante, Olaf

Prasad, Vikas

Prasanna, Prateek

Pravettoni, Gabriella

Prawitt, Dirk

Preca, Bogdan Tiberius

Preda, Lorenzo

Prehn, Jochen

Prehn, Jochen H. M.

Preti, Mario
Preudhomme, Claude

Prevarskaya, Natalia

Previs, Rebecca A.

Prezioso, Lucia

Prigerson, Holly G.

Primignani, Massimo

Prince, Mark E.

Prise, Kevin M.

Pritchard, Catrin

Privat-Maldonado, Angela

Privette Vinnedge, Lisa

Proescholdt, Martin

Provencio, Mariano

Pryczynicz, Anna

Przemek, Krawczyk

Psoroulas, Serena

Psyrri, Amanda

Pudakalakatti, Shivanand M.

Pugliese, Mariagabriella

Puhka, Maija

Puig-Butillé, Joan Anton

Puijk, Robbert

Puisset, Florent

Pula, Bartosz

Puladi, Behrus

Puliani, Giulia

Pulido, Rafael

Pulito, Claudio

Pulliero, Alessandra

Pusch, Stefan

Puustinen, Pietri

Puy, Cristina

Pylayeva-Gupta, Yuliya

Pyronnet, Stéphane

Qaddoumi, Ibrahim

Qdaisat, Aiham

Qi, Xiaoyang

Qi, Yanfei (Jacob)

Qi, Yue

Qian, Yanrong

Qiao, Fangfang

Qin, Weijun

Qin, Zhiqiang

Qing, Tao

Qiu, Bin

Qiu, Guangyu

Qiu, Jiantai Timothy

Qiu, Zhen

Qu, Ning

Qu, Peng

Quadri, Marika 
Quadri, Syed A.

Quagliariello, Vincenzo

Quaia, Emilio

Quante, Michael

Quarta, Alessandra

Quatrini, Linda

Quattro, Joseph M.

Quax, Paul

Quax, Wim J.

Que, Jenny

Quek, Camelia

Quiding-Järbrink, Marianne

Quinn, Gwendolyn

Quinn, Gwendolyn P.

R. Naik, Akshata

Raber-Durlacher, Judith E.

Rabien, Anja

Rada, Miran

Radeghieri, Annalisa

Rades, Dirk

Radisavljevic, Ziv

Radochová, Vladimíra

Radosavljević, Tatjana

Radosevic-Robin, Nina

Radu, Beatrice

Radulovic, Marko

Radziejewska, Iwona

Radzki, Dominik

Rafael, Diana

Rafaelsen, Søren Rafael

Raffone, Antonio

Raghavendra, Akshara

Ragno, Pia

Ragoussis, Jiannis

Raguse, Jan-Dirk

Rahimi, Siavash

Rahman, Arman

Rahman, Nafis

Rahmani, Redi

Rai, Ganesh

Rai, Khushminder

Rai, Vikrant

Raia, Maddalena

Raikhy, Gaurav

Raimondo, Diego

Raja, Iruthayapandi Selestin

Rajagopalan, Anugraha

Rajasekaran, Narendiran

Rajendran, Praveen

Raji, Idris

Rajkumar Singh, Kalra
Rajpurohit, Surendra

Rakislova, Natalia

Ramakrishnan, Vijay

Ramasamy, Mohankandhasamy

Ramazanov, Bulat

Rambaldi, Alessandro

Rameshwar, Pranela

Ramírez, Cristina M.

Ramírez-Labrada, Ariel

Ramirez-Peña, Esmeralda

Ramon Torrell, Josep Maria

Ramos, Emilio

Ramos-Garcia, Pablo

Rampazzo, Elena

Rampias, Theodoros

Ramsköld, Daniel

Randazzo, Antonio

Ranganna, Kasturi

Rangel-Pozzo, Aline

Rani Banik, Gouri

Ranieri, Danilo

Ranieri, Girolamo

Ranjit, Sabina

Ransac, Stéphane

Rantala, Juha K.

Rao, Geeta

Rao, Mahadev

Rao, Rajini

Rao, Tata Nageswara

Raoul, William

Rapi, Stefano

Rapic, Sara

Rapino, Cinzia

Rapisarda, Venerando

Rasche, Leo

Raschke, Felix

Raška, Milan

Rasmussen, Torben Riis

Rasouly, Hila Milo

Raspagliesi, Francesco

Rasul, Sazan

Ratajska, Magda

Rather, Gulam

Rathert, Philipp

Ratti, Stefano

Rattray, Zahra

Raundhal, Mahesh

Raup-Konsavage, Wesley

Rausei, Stefano

Rauso, Raffaele

Rav Acha, Moshe 
Rava, Marta

Ravaioli, Matteo

Ravanelli, Marco

Rawat, Manmeet

Rawicz-Pruszyński, Karol

Ray, Arghya

Ray, Upasana

Rayar, Michel

Razzokov, Jamoliddin

Razzuoli, Elisabetta

Rbah-Vidal, Latifa

Read, Matthew

Reader, Jocelyn

Reagan, John L.

Reali, Eva

Reddick, Wilburn E.

Reddy Bonam, Srinivasa

Reddy, Aswin

Reddy, Kesava

Reddy, Sudhir Putty

Redina, Olga

Redini, Francoise

Redondo Muñoz, Javier

Reed, Amy McCart

Reese, Jennifer Barsky

Reeves, Helen L.

Regel, Ivonne

Reggiani, Francesca

Reginato, Mauricio

Regnery, Sebastian

Rehfeld, Jens Frederik

Reich, Adam

Reich, Waldemar

Reig, Òscar

Reimer, Daniel U.

Reinders, Joerg

Reindl, Katie

Reiner, Anne S.

Reinhardt, Heike

Reis, Rui Manuel

Reis, Sara S.

Ren, Xiang

Rengarajan, Venkatakrishnan

Renzo, Vanna

Resheq, Yazid J.

Resnick, Igor

Restaino, Stefano

Retsky, Michael

Rettig, Eleni

Rettinger, Eva

Reu, Frederic J.
Revelli, Luca

Revuri, Vishnu

Reyes-Aldasoro, Constantino Carlos

Reymond, Marc

Reyners, Anna K. L.

Rho, Jin Kyung

Rialland, Mickaël

Ribatti, Domenico

Ribero, Simone

Ricardo, Sara

Ricchetti, Francesco

Ricci, Alberto

Ricci, Angela Dalia

Ricci, Francesca

Ricci, Giovanna

Ricci, Giulia

Ricciardi, Maria Rosaria

Rich, Thomas C.

Richardson, Alan

Richardson, Sandra R.

Riches, John C.

Richmon, Jeremy

Richter, Günther H. S.

Richter, Susan

Ricke, William

Rider, Paul

Ridola, Lorenzo

Ridolfi, Laura

Riefolo, Mattia

Riegman, Peter H. J.

Rieken, Stefan

Riether, Carsten

Rigacci, Luigi

Rigalli, Juan Pablo

Rigaud, Michel

Rigby, Matthew H.

Rigopoulou, Eirini

Riis, Margit L. H.

Rim, Chai Hong

Rimondini, Lia

Ringdén, Olle

Riond, Joëlle

Riou, Olivier

Riquelme, Arnoldo

Rishi, Arun

Riva, Giulia

Riva, Giuseppe

Rivero, Francisco

Rizcallah, Edmond

Rižner, Tea Lanišnik

Rizzo, Alessandro 
Rizzo, Angela

Rizzolio, Flavio

Roberg, Karin

Roberts, David D.

Roberts, Janet

Roberts, Tara

Robichaux, Jacqulyne

Robinson, James

Robitaille, Mélanie

Roccaro, Aldo

Rocco, Gaetano

Rochani, Ankit

Roche, Serge

Rodeberg, David

Roderburg, Christoph

Rodland, Karin D.

Rodolfo, Monica

Rodrigo, Juan P.

Rodrigues Santiago, Leandro

Rodrigues, Dario

Rodrigues, Pedro Miguel

Rodríguez, Cristina

Rodriguez, F. David

Rodríguez, Javier

Rodríguez-García, Alba

Rodriguez-Lafrasse, Claire

Rodriguez-Nogales, Carlos

Rodríguez-Santamarta, Tanía

Rodriguez-Vargas, Jose Manuel

Roedel, Franz

Roels, Juliette

Roesch, Alexander

Roessner, Eric

Rofi, Eleonora

Rogakou, Emmy

Rogalska, Aneta

Rogatto, Silvia

Rogowski, Paul

Roh, Tae Hoon

Rohde, Stefan

Röhrlich, Pierre Simon

Rojiani, Mumtaz V.

Rojo, Jose M.

Rok, Jakub

Rokavec, Matjaz

Rola, Radosław

Roll, Wolfgang

Romagnoli, Stefano

Roman, Maciej

Romani, Andrea

Romanini, Antonella
Romano, Alberto

Romanov, Victor

Roma-Rodrigues, Catarina

Rombouts, Krista

Romee, Rizwan

Romero Collado, Angel

Romero-Masters, James

Romeyke, Tobias

Romiti, Giulio Francesco

Rommelaere, Jean

Rompianesi, Gianluca

Róna, Gergely

Ronchetti, Domenica

Ronellenfitsch, Ulrich

Rong, Chan Kuan

Rong, Ruichen

Ronot, Maxime

Roodhart, Jeanine

Roper, Nitin

Roque, Natalia

Ros, Javier

Ros, Susana

Rosa-Caldwell, Megan E.

Rosado, Juan

Rosahl, Steffen

Rosanò, Laura

Roschke, Anna

Rose, Michael

Roseline, Godbout

Rosen, Theodore

Rosenbaum, Thorsten

Rosenberg, Jens T .

Roshan, Amit

Rosol, Thomas

Ross, Jeffrey S.

Rossi, Francesca

Rossi, Maija

Rossi, Paolo

Rossi, Roberta Elisa

Rossiello, Francesca

Rossini, Daniele

Rosswog, Carolina

Rossy, Jérémie

Rotblat, Barak

Rothberg, Paul

Rotte, Anand

Roulois, David

Roumeguère, Thierry

Rousseau, Audrey

Rousselle, Patricia

Rovida, Elisabetta 
Rovirosa, Ángeles

Rovo, Alicia

Roy, Soumyajit D.

Royer-Pokora, Brigitte

Royse, Kathryn E.

Rozman, Aleš

Rual, Jean-Francois

Rubben, Albert

Rubio Retama, Jorge

Rubis, Blazej

Rubnitz, Jeffrey E.

Rubtsova, Maria P.

Ruchała, Marek

Rudd, Sean G

Rudelius, Martina

Rudolf, Ruediger

Rueda, Bo

Ruehl, Heiko

Ruggeri, Rosaria M.

Ruggiero, Maria Rosaria

Ruhaak, Lucia Renee

Ruiz, Facundo

Ruiz, Irene Rius

Rumpold, Holger

Rund, Deborah

Ruotsalainen, Janne

Rusak, Malgorzata

Rusanova, Iryna

Russell, Nicola

Russell, Stepehen

Russo, Alessandro

Russo, Angela

Russo, Antonio

Russo, Daniela

Russo, Giorgio

Russo, Giovanna

Russo, Isabella

Ruszkowska-Ciastek, Barbara

Rutkowsk, Piotr

Ruvo, Menotti

Ruzgys, Paulius

Ruzov, Alexey

Ruzzene, Maria

Ruzzenente, Andrea

Ryan, Aideen

Rydén, Lisa

Ryu (Yoo), Kyung Hyun

Ryu, Bomi

Ryu, Jung Su

Ryu, Keun Ho

Rzepakowska, Anna
Rzymowska, Jolanta

Saar, Matthias

Sabaawy, Hatem E.

Sabarimurugan, Shanthi

Sabatier, Laure

Sabatier, Renaud

Sabatino, Giovanni

Sabloff, Mitchell

Sabol, Maja

Sacchini, Virgilio

Saccilotto, Ramon

Saccone, Salvatore

Saceda, Miguel

Sack, Ulrich

Saddik, Basema

Sadhukhan, Pritam

Sadler, Anthony

Saeki, Issei

Safa, Ahmad R.

Safi, Seyer

Safránek, David

Saglio, Francesco

Sagnou, Marina

Saha, Biswarup

Saha, Nirmalya

Saha, Shekhar

Sahara, Hiroeki

Sahgal, Pranshu

Sahu, Sanjaya Kumar

Saillard, Charlie

Saini, Sharanjot

Saito, Ryoko

Saito, Ryuta

Saito, Tsuyoshi

Saito, Yuichi

Saitoh, Takayuki

Sajesh, Babu V.

Sajjadi, Elham

Sakai, Kazuko

Sakakura, Noriaki

Sakamoto, Shinichi

Sakamoto, Yasunari

Sakamoto, Yuzuru

Sakamuro, Daitoku

Sakata, Kiyohiko

Sakuma, Kunihiro

Sakwe, Amos

Salagierski, Maciej

Salar, Antonio

Salas, Lucas A.

Sałat, Kinga 
Salazar, Ivan

Saleh, Fatima A.

Saleh, Mazen

Salehzadeh-Yazdi, Ali

Salgar, Shashikumar

Salhi, Yahia

Salido, Marta

Salimi, Marzieh

Salla, Mohamed

Salle, Valéry

Salomé Pires, Ana

Salomon, Carlos

Salto-Tellez, Manuel

Salvatore, Lisa

Salvatori, Pietro

Samaja, Michele

Samalin, Emmanuelle

Samaniego, Rafael

Samarzija, Ivana

Samasca, Gabriel

Sambri, Andrea

Samec, Marek

Sammarco, Giuseppe

Samnick, Samuel

Samuels, Brian

Samykutty, Abhilash

Sanawar, Rahul

Sánchez-Beato, Margarita

Sánchez-Bueno, Francisco

Sanchez-Elsner, Tilman

Sánchez-Pérez, Isabel

Sancisi, Valentina

Sanda, Miloslav

Sandfeld-Paulsen, Birgitte

Sandhu, Shahneen K.

Sanduleanu, Silvia

Sanguedolce, Francesca

Sanità, Gennaro

Sano, Makoto

Sansom-Daly, Ursula

Sansone, Anna

Sansone, Francesco

Santamaria-Martínez, Albert

Santarelli, Andrea

Santini, Donatella

Santonico, Elena

Santos, Joana M. O

Santos, Mafalda

Santos, Mirentxu

Santos-Lozano, Alejandro

Santos-Rocha, Rita Alexandra
Santpere, Gabriel

Santulli, Gaetano

Sanz, Miguel A.

Sapalidis, Konstantinos

Saponaro, Concetta

Sarabia-Cobo, Carmen María

Sarantis, Panagiotis

Sardella, Eloisa

Sargi, Zoukaa

Sarhadi, Virinder Kaur

Sarhan, Dhifaf

Sarkadi, Balazs

Sarkar, Anujit

Sarkar, Sibaji

Sarkozy, Clémentine

Sarma, Pranjal

Sarmento-Ribeiro, Ana Bela

Sarna, Michal

Sarno, Federica

Sarobe, Pablo

Sarosiek, Shayna R.

Sarrette, Jean Philippe

Sarria, Gustavo R.

Sartor, Maureen A.

Sasada, Tetsuro

Sasai, Noriaki

Sasaki, Makoto

Sasaki, Motoko

Sasaki, Yasushi

Sascha, Rahn

Sasso, Ferdinando Carlo

Sastre-Serra, Jorge

Sathiyaraj, Srinivasan

Sato, Fuyuki

Sato, Hiroki

Sato, Mariko

Sato, Shuichi

Sato, Yukiyasu

Sato, Yusuke

Satoh, Taroh

Satoi, Sohei

Saulnier, Olivier

Saulnier, Ron

Sauro, Khara

Sauter, Edward R.

Savic, Lynn Jeanette

Savic-Radojevic, Ana

Savvidou, Ioanna

Sawabata, Noriyoshi

Sawada, Koji

Sawyer, Elinor J. 
Sayed, Ibrahim M.

Scaife, Courtney L.

Scala, Stefania

Scalabrin, Mattia

Scalco, Elisa

Scarfò, Lydia

Scarpa, Edoardo

Scarpi, Emanuela

Scatena, Roberto

Schache, Andrew

Schäfer, Georgia

Schäfer, Markus

Schaiquevich, Paula

Scharovsky, Olga Graciela

Schattner, Mark A.

Scheffler, Matthias

Scheie, David

Scheijen, Blanca

Scheipl, Susanne

Schell, Michael

Schengrund, Cara-lynne

Schenke, Simone

Scherer, Dominique

Scherthan, Harry

Scherwath, Angela

Scherz-Shouval, Ruth

Schiavello, Elisabetta

Schiavetti, Amalia

Schiavina, Riccardo

Schiavone, Nicola

Schiavoni, Giovanna

Schick, Joel A.

Schierz, Oliver

Schildgen, Olivier

Schildgen, Verena

Schildkraut, Joellen M.

Schilling, Clare

Schindlbeck, Christian

Schinke, Carolina D.

Schinke, Thorsten

Schiødt, Frank Vinholt

Schioppa, Tiziana

Schirmer, Bastian

Schirren, Rebekka

Schlack, Katrin

Schlaepfer, Isabel

Schlumbrecht, Matthew

Schmeel, Leonard Christopher

Schmelz, Eva M.

Schmid, Evi

Schmid, Thomas E.
Schmidl, Doreen

Schmidt, Henrik

Schmidt, Manfred

Schmidt, Marcus

Schmidt, Peter Thelin

Schmidtke, Gunter

Schmitt, Fernando

Schmitt, Nicole

Schmitz, Marc

Schmitz, Ulf

Schmitz-Dräger, Bernd J.

Schneider, Gabriela

Schneider-Stock, Regine

Schnütgen, Frank

Schoeftner, Stefan

Schoffer, Olaf

Schonfeld, Sara

Schönthal, Axel H.

Schorb, Elisabeth

Schrader, Jörg

Schrefler, Bernhard

Schrump, David S.

Schueler, Julia

Schug, Zachary

Schuler, Linda

Schuler, Patrick

Schuller, Hildegard M.

Schulte, Fiona

Schulte, Reinhard

Schulz, Wolfgang A.

Schulze-Bergkamen, Henning

Schuringa, Jan Jacob

Schuster, Iona

Schwarz, Herbert

Schwarzbacherová, Viera

Schwind, Sebastian

Scimeca, Manuel

Scioli, Maria Giovanna

Scomparin, Anna

Sconocchia, Giuseppe

Scott, Alasdair

Scott, Jeffrey F.

Scott, Rodney J.

Seano, Giorgio

Sebastianelli, Arcangelo

Sebens, Susanne

Sebestyén, Anna

Secomb, Timothy W.

Secrier, Maria

Sedykh, Sergey

See, Kay Choong 
Seger, Rony

Segovia, Cristina

Ségui, Bruno

Seguin, Laetitia

Segura, Miguel F.

Seibel, Nita L.

Seidl, Maximilian

Seidlits, Stephanie

Seif, Alix E.

Seith, Ferdinand

Sejda, Aleksandra

Sekino, Yohei

Seliger, Corinna

Seligson, Nathan

Selingerova, Iveta

Selivanova, Svetlana V.

Selleri, Carmine

Sellitto, Assunta

Semczuk, Andrzej

Semedo, Teresa

Semenzato, Gianpietro

Sengal, Asmerom

Sengodan, Satheesh

Šenigl, Filip

Senkal, Can

Serafini, Simone

Sergi, Pier Nicola

Serio, Gabriella

Sério, Susana

Serra, Raffaele

Serrano-Pertierra, Esther

Serratì, Simona

Serša, Igor

Servais, Stephane

Setaluri, Vijayasaradhi

Sethunath, Vidyalakshmi

Settembre, Carmine

Sevenich, Lisa

Severi, Stefano

Severin, Filippo

Sevigny, Mary

Seyfried, Steffen

Seymour, John F.

Sfacteria, Alessandra

Shabason, Jacob E.

Shackelford, Julia

Shah, Girish M.

Shah, Jainil

Shah, Ruchi

Shahbaaz, Mohd

Shahriyari, Leili
Shaik, Shahensha

Shaikh, Mushfiq

Shajahan-Haq, Ayesha N.

Shakeel, Faiyaz

Shamay, Yosi

Shammas, Masood

Shan, Yi

Shankar, Eswar

Shankargouda, Patil

Shanmugam, Vignesh

Shao, Jieya

Shao, Lijian

Shao, Yanjiao

Shao, Yu-Hsuan Joni

Sharif, Jafar

Sharker, Shazid Md.

Sharma, Ajay

Sharma, Anand

Sharma, Bhesh Raj

Sharma, Geeta

Sharma, Nikita

Sharma, Piyush

Sharma, Shweta

Sharonov, George Vladimirovich

Shaw, Priyanka

Shehata, Mohamed

Sheikh, Nadeem

Shen, Che-Hung

Shen, Guodong

Shen, Shiqian

Shen, Yu

Sheng, Yue

Sher, Yuh-Pyng

Sherbenou, Daniel W.

Sherif, Amir

Sherwani, Mohammad Asif

Sheshadri, Namratha

Shetty, Mihir

Sheu, Jim

Shi, Di

Shi, Hon-Yi

Shi, Jian

Shi, Kuangyu

Shi, Lei

Shi, Lewis

Shi, Min

Shiah, Shine-Gwo

Shiba, Kiyotaka

Shibata, Yasushi

Shidoji, Yoshihiro

Shieh, Dar-Bin 
Shigeta, Kohei

Shih, Jing-Wen

Shiina, Marisa

Shimoda, Mitsugi

Shimodaira, Shigetaka

Shimojo, Masahito

Shimokawa, Tsuneo

Shimoni, Avichai

Shimose, Shigeo

Shin, Daniel Sanghoon

Shin, Vivian Yvonne

Shinde, Aparna

Shinji, Kawabata

Shinohara, Hisashi

Shinohara, Shogo

Shinomiya, Nariyoshi

Shintani, Yasushi

Shiota, Seiji

Shioyama, Yoshiyuki

Shirabe, Ken

Shiradkar, Rakesh

Shirai, Keisuke

Shiraishi, Kenshirou

Shiraishi, Kouya

Shirato, Hiroki

Shirole, Nitin

Shiue, Yow-Ling

Shivakumar, Pranav

Shnyder, Steve

Shojaei, Shahla

Shoji, Sunao

Shore, Neal

Shoshani, Ofer

Shoumariyeh, Khalid

Shpacovitch, Victoria

Shramova, Elena Ivanovna

Shrestha, Dhan

Shrestha, Hridaya

Shu, Sherry

Shuboni-Mulligan, Dorela

Shukla, Kirtikar

Shukla, Siddharth

Shuo, Qie

Shurin, Michael

Sibon, David

Sibson, David Ross

Siddique, Abu Bakar

Sidhu, Stan B.

Sieber, Oliver

Siegel, Peter

Siemer, Stefan
Siena, Salvatore

Sieni, Elisabetta

Sierżęga, Marek

Sifrer, Robert

Signorelli, Christina

Sikic, Danijel

Sikora, Ewa

Sikov, William M.

Silic-Benussi, Micol

Sillerud, Laurel O.

Silva, Ana Luísa

Silva, Catarina

Silva-Rodríguez, Jesús

Silver, Daniel J.

Silverman, Michael J.

Silvestri, Giovannino

Silvestri, Marco

Silvestri, Valentina

Silvestris, Nicola

Sim, Sung Hoon

Simão, André L.

Simeone, Claudio

Simeone, Pasquale

Simionescu, Natalia

Simon, Christian

Simon, Thomas

Simona, Kranjc

Simons, Brian Wesley

Simpson, Fiona

Sims, Jennifer

Sinescu, Ruxandra Diana

Singer, Eric A.

Singer, Stephan

Singh, Ajay Vikram

Singh, Anurag K.

Singh, Chandra

Singh, Deepak

Singh, Neha

Singh, S. K.

Singh, Santosh Kumar

Singh, Sarbjit

Singh, Shilpa

Singh, Sneha

Singh, Vivek Kumar

Singhal, Sandeep K.

Sinha, Birandra K.

Sinha, Debottam

Sinha, Divya

Sinha, Raghu

Sini, Maria Cristina

Sinnberg, Tobias W. 
Sioud, Mouldy

Sipos, Ferenc

Sippl, Wolfgang

Siraj, Afjalus

Siravegna, Giulia

Sirinian, Chaido

Sirvent, Audrey

Sitar, Daniel S.

Sitbon, Marc

Sitia, Leopoldo

Siu, Michelle K. Y.

Sivakumar, Sushama

Siwko, Stefan

Sjöberg, Rickard L.

Skardelly, Marco

Skiecevičienè, Jurgita

Skoda, Jan

Skonieczna, Magdalena

Skov, Vibe

Skrap, Miran

Skręt, Andrzej

Skrzypczak, Maciej

Skrzypek, Klaudia

Skvortsova, Ira-Ida

Slade, Neda

Slobodin, Boris

Slominski, Andrzej

Slomovitz, Brian M.

Słowińska-Klencka, Dorota

Smaglo, Brandon G.

Smahel, Michal

Smetana, Karel

Śmieszek, Agnieszka

Sminia, Peter

Smith, Deanna S.

Smith, Eric

Smolarczyk, Ryszard

Smolle, Josef

Smrž, Daniel

Snijders, Tom J.

Snow, Stephanie

Snyder, Christopher

So, Jonathan

Soares, Paula

Soave, Armin

Sobas, Marta

Sobhani, Navid

Sobiak, Joanna

Sobolewski, Cyril

Sobrinho-Simões, Manuel

Socea, Bogdan
Socha, Małgorzata

Socorro, Silvia

Soddu, Silvia

Sodergren, Samantha C.

Soejima, Toshinori

Soffietti, Riccardo

Sogawa, Rintaro

Sohrabi, Amir

Soibam, Benjamin

Solana, Rafael

Solanki, Sumeet

Solbiati, Luigi

Solé Cañadas, Carla

Solimando, Antonio G.

Solinas, Giuliana

Solip, Park

Solli, Piergiorgio

Soltész, Beáta

Somasundaram, Ashwin

Son, Jaekyoung

Sondak, Vernon

Sondarva, Gautam

Sone, Hideko

Song, Chang W.

Song, Changhoon

Song, Guisheng

Song, Jaewhan

Song, Minjung

Song, Xinni

Soni, Aashish

Sonis, Stephen

Sonowal, Himangshu

Soon, Hong

Soond, Surinder M.

Soons, Zita

Sørensen, Anne Vest

Sorensen, Poul H.

Sorigué, Marc

Sorolla, Maria Alba

Soror, Tamer Y.

Sorrenti, Salvatore

Sorrentino, Rosalinda

Sosnowska-Pasiarska, Barbara

Sossey-Alaoui, Khalid

Sotirchos, Vlasios

Sotomayor, Camilo G.

Souers, Andrew

Souglakos, John

Soulen, Michael C.

Sounni, Nor Eddine

Sousa, Angela 
Southall, Noel

Souwer, Esteban T. D.

Sp, Nipin

Spaargaren, Marcel

Spadarella, Gaia

Spagnolo, Francesco

Spałek, Mateusz

Spałek, Mateusz Jacek

Span, Paul

Spanakis, Marios

Spanheimer, Philip

Spathis, Aris

Speeckaert, Marijn

Sperling, Adam

Sperti, Cosimo

Spiazzi, Luigi

Spindler, Karen Lise Garm

Spinelli, Laura

Spinnato, Paolo

Spirina, Liudmila V.

Spoerri, Loredana

Spoletini, Gabriele

Sprave, Tanja

Sprengers, Dave

Spring, Kevin

Sreedharan, Sreejesh

Srikanthan, Amirrtha

Srivastava, Ankit

Srivastava, Anuj

Srivastava, Swayam Prakash

Sroka, Ronald

Staber, Philipp Bernhard

Stack, Sharon

Stadlbauer, Andreas

Stafford, Francis William

Stål, Per

Stålberg, Peter

Stalpers, Lukas

Stanganelli, Ignazio

Stanislawska-Sachadyn, Anna

Stannula, Martin

Stanzione, Arnaldo

Starzyńska, Anna

Stathopoulos, Constantinos

Stati, Valeria

Stauber, Roland

Staudenherz, Anton

Stawski, Robert

Stecca, Tommaso

Steckiewicz, Karol

Steeghs, Neeltje
Steenblock, Charlotte

Steenbrugge, Jonas

Stefano, Alessandro

Stefanowicz, Joanna

Stefanska, Anna

Steinbach, Joachim

Steinbrunn, Torsten

Steinemann, Doris

Steinkamp, Mara P.

Stella, Alessandro

Stella, Giulia M.

Stella, Stefania

Stelmes, Jean-Jacques

Stemmler, Marc

Stenman, Adam

Stenzl, Arnulf

Stepanenko, Aleksei A.

Stepanov, Alexey V.

Stephen, Andrew G.

Stepp, Herbert

Stergios, Konstantinos

Steri, Veronica

Sterpetti, Antonio V.

Stewart-Ornstein, Jacob

Stirke, Arunas

Stobiecka, Magdalena

Stock, Christian

Stockmann, Christian

Støer, Nathalie C.

Stoian, Dana

Stoklosa, Tomasz

Stoppelli, Maria Patrizia

Storey, Kathleen

Störmann, Sylvère

Stovell, Matthew

Strand, Elin

Strano, Sabrina

Strati, Areti

Stratikos, Efstratios

Straus, David J.

Stravopodis, Dimitrios J.

Streba, Costin Teodor

Strigari, Lidia

Strippoli, Raffaele

Ströbel, Philipp

Strocchio, Luisa

Strowitzki, Moritz

Strub, Thomas

Štrumfa, Ilze

Strus, Magdalena

Strzelczyk, Joanna 
Stuart, Heather

Stuppia, Liborio

Sturtzel, Caterina

Stüssi, Georg

Su, Lydia Min-Ying

$\mathrm{Su}, \mathrm{Rui}$

Su, Shih-bin

$\mathrm{Su}$, Wen-Pin

$\mathrm{Su}$, Xinming

$\mathrm{Su}$, Ying-Hsiu

Suarez-Cunqueiro, María Mercedes

Subirán, Nerea

Suckow, Mark

Suda, Kenichi

Sudbery, Ian

Suehara, Yoshiyuki

Suematsu, Makoto

Sugaya, Makoto

Sugie, Tomoharu

Sugimoto, Mitsushige

Sugimura, Rio Ryohichi

Suh, Chong Hyun

Suh, Yunsuhk

Suhail, Yasir

Suijkerbuijk, Karijn

Sukhanova, Mariya V.

Sukhorukov, Vladimir

Sukowati, Caecilia H. C.

Sulová, Zdenka

Summers, Yvonne Jane

Sun, Duanping

Sun, Jianmin

Sun, Jing

Sun, Ramon

Sun, Yilun

Sun, Yin

Sun, Zhonghua

Sunami, Yoshiaki

Sundararajan, Kousik

Sundararajan, Vignesh

Sundín, Anders E.

Sundvall, Maria

Sung, Kyung Hyun

Sung, Wen-Wei

Supernat, Anna

Surdutovich, Eugene

Sureda, Anna

Șurlin, Valeriu Marin

Surov, Alexey

Suryawanshi, Amol

Suso, Else Marit Inderberg
Sutherland, Kate

Suwan, Keittisak

Suzuki, Hajime

Suzuki, Kazuhito

Suzuki, Shinichi

Svahn, Tony Martin

Svendsen, Lars Bo

Sverdlov, Aaron

Sverrisdóttir, Eva

Swain, Sandra M.

Swami, Umang

Świerczewska, Monika

Swijnenburg, R. J.

Sykiotis, Gerasimos P.

Szabo, Ildiko

Szanto, Ildiko

Szarvas, Tibor

Szaryńska, Magdalena

Szatmári, Dávid

Szeliga, Monika

Szijan, Irene

Szilágyi, Melinda

Szmajda-Krygier, Dagmara

Szolnoky, Győző

Szoor, Arpad

Szturz, Petr

Szuhai, Karoly

Szumera-Cieckiewicz, Anna

Szyc, Łukasz

Szymanski, Wiktor

Szymczak-Pajor, Izabela

Tabarkiewicz, Jacek

Tabatabai, Ghazaleh

Tabish, Tanveer Ahmad

Taboni, Stefano

Tacconelli, Stefania

Tada, Toshifumi

Tafuto, Salvatore

Taghizadeh, Hossein

Tagliabue, Elda

Tagliabue, Marta

Tagliati, Corrado

Taieb, Julien

Takabatake, Kiyofumi

Takada, Yasutsugu

Takagi, Kiyoshi

Takahama, Takayuki

Takahashi, Chiaki

Takahashi, Hiroyuki

Takahashi, Mamoru

Takahashi, Shyunji 
Takai, Atsushi

Takam Kamga, Paul

Takamaru, Hiroyuki

Takaori-Kondo, Akifumi

Takasawa, Ken

Takashi, Sekiya

Takayama, Kenichi

Takeda, Hiroyuki

Takeda, Masayuki

Takeda, Toshikazu

Takei, Hiroyuki

Takenaka, Mamoru

Talar-Wojnarowska, Renata

Talesa, Vincenzo Nicola

Tallet, Agnès V.

Talos, Flaminia

Talukdar, Fazlur Rahman

Talukdar, Sarmistha

Tam, Shing Yau

Tam, Shing Yau Marco

Tamagno, Ilaria

Tamaian, Radu

Tambuwala, Murtaza

Tampe, Björn

Tamura, Hideto

Tamura, Masaaki

Tamura, Ryota

Tamura, Yasuaki

Tamvakopoulos, Constantin

Tan, Karen

Tan, Li

Tan, Nguan Soon

Tan, Tuan Zea

Tan, Yuting

Tan, Zhengguo

Tanabe, Hiroki

Tanabe, Shihori

Tanaka, Nobuyuki

Tanaka, Yuka

Tanda, Maria Laura

Tang, Kwan Ho

Tang, Patrick Ming-Kuen

Tang, Yuting

Tangherloni, Andrea

Tani, Kenzaburo

Tanic, Nikola

Tanino, Hirokazu

Tanioka, Maki

Taniwaki, Masafumi

Taniyama, Daiki

Tantos, Ágnes
Taraboletti, Giulia

Tarallo, Roberta

Tarantino, Giovanni

Tarn, Woan-Yuh

Tarnowski, Maciej

Tartaglia, Francesco

Tartarone, Alfredo

Tartey, Sarang

Tartour, Eric

Taschner-Mandl, Sabine

Tashima, Toshihiko

Tashireva, Liubov A.

Tashiro, Haruko

Tatić, Svetislav

Tátrai, Péter

Taube, Joseph

Taubenberger, Anna

Taulier, Nicolas

Tavernier, Jan

Taylor, Justin W.

Taylor, Matthew

Taylor, Renea A.

Taylor, Verdon

Tazzari, Marcella

Tazzioli, Giovanni

Tchoghandjian-Auphan, Aurélie

Tebbi, Cameron $\mathrm{K}$.

Teicher, Beverly A.

Teijeira, Álvaro

Teisseyre, Andrzej

Teixidó, Cristina

Tejero, Jesús

Tellez Gabriel, Marta

Tellier, Michael

Telukutla, Srinivasa Reddy

Temel, Sehime

Temraz, Sally

Ten Hacken, Elisa

Tenenbaum, Scott

Teng, Chieh-Lin

Teng, Jian

Teng, Wei

Teoh-Fitzgerald, Melissa

Terai, Mizue

Terao, Mineko

Teras, Lauren R.

Terazono, Hideyuki

Teresa, Calimeri

Terp, Mikkel Green

Terry, Paul D.

Terry, Samantha 
Terry, Stéphane

Terry, Stephen J

Terstappen, Leon

Terzuoli, Lucia

Tesson, Mathias

Tessoulin, Benoit

Testori, Alessandro A. E.

Teterycz, Paweł

Teusch, Nicole

Thakur, Abhimanyu

Thakur, Shilpa

Thalhammer, Theresia

Thangavel, Chellappagounder

Tharehalli, Umesh

Theil, Gerit

Theillet, Charles

Thellung, Stefano

Themistocleous, Marios

Thevenod, Frank

Thevenot, Paul T.

Thijssen, Rachel

Thomas, Giju

Thomassen, Mads

Thomsen, Caroline Emilie Brenner

Thomsen, Martin K.

Thomsen, Thomas

Thong, Melissa Suk Yin

Thorenoor, Nithyananda

Thurber, Greg M.

Thurston, David E.

Thuru, Xavier

Thyagarajan, Bharat

Tiberio, Guido Alberto Massimo

Ticconi, Carlo

Tiller, Jane

Tilvawala, Ronak

Timchenko, Nikolai

Timo, Carpén

Timofeev, Oleg

Timoshenko, Viktor

Tinari, Nicola

Tinelli, Andrea

Ting, Wen-Chien

Tinguely, Marianne

Tiribelli, Claudio

Tiribelli, Mario

Tiriveedhi, Venkataswarup

Tischler, Arthur

Tittarelli, Andrés

Tixeira, Rochelle

Tixier, Florent
Tiziani, Stefano

Tjahyono, Daryono Hadi

Tobiume, Kei

Toda, Takashi

Todaro, Matilde

Toden, Shusuke

Todoerti, Katia

Tofanelli, Margherita

Tognolatti, Piero

Toh, Tan Boon

Tohru, Morisada

Toillon, Robert-Alain

Toivanen, Roxanne

Tokino, Takashi

Tokumaru, Sunao

Tokumaru, Yoshihisa

Tokunaga, Hideki

Tokushige, Katsutoshi

Toledano, Michel B.

Toledo, Franck

Tolosano, Emanuela

Tomaiuolo, Rossella

Tomasetto, Catherine

Tomasetto, Catherine-Laure

Tomasi, Maria Lauda

Tomašič, Tihomir

Tomasini, Richard

Tomasson, Michael

Tomaszkiewicz, Marta

Tometich, Danielle

Tomić, Josip

Tominaga, Naoomi

Tomoaki, Okimoto

Tomuleasa, Ciprian

Tonacci, Alessandro

Tonelli, Francesco

Tong, Jessica

Tong, Man

Tordai, Attila

Tordjman, Karen M.

Torigoe, Toshihiro

Torimura, Takuji

Tornesello, Anna Lucia

Tornillo, Giusy

Tornin, Juan

Torrisani, Jérôme

Torrisi, Rosalba

Torstein, Meling

Toruner, Gokce Altay

Toschi, Elena

Toshito, Toshiyuki 
Tosti, Giulio

Toth, Reka

Toti, Paolo

Totonchy, Jennifer

Totta, Pierangela

Tournigand, Christophe

Toutain, Jérôme

Touw, Ivo P.

Tovoli, Francesco

Townsend, Julie

Toyama-Sorimachi, Noriko

Trader, Darci

Trainer, Alison $\mathrm{H}$.

Trama, Annalisa

Trapani, Dario

Traub, Frank

Traverso, Alberto

Treacy, Oliver

Treglia, Giorgio

Trembley, Janeen

Tretter, Laszlo

Trevisani, Franco

Trevisi, Gianluca

Triche, Timothy

Trikalinos, Nikolaos

Trimboli, Pierpaolo

Triner, Daniel

Tripathi, Madhulika

Tripathi, Rakshamani

Tripon, Florin

Trivedi, Rachana

Troadec, Marie-Bérengère

Troconiz, Iñaki F.

Trojani, Alessandra

Troschel, Fabian

Troschel, Fabian Martin

Trotta, Rossana

Trovato, Maria

Trufero, Javier Martínez

Truillet, Charles

Truksa, Jaroslav

Tsagozis, Panagiotis

Tsai, Chi-Neu

Tsai, Hung-Wen

Tsai, I-Lin

Tsai, Jo-Ting

Tsai, Ping-Hsing

Tsai, Shih-chang

Tsai, Wan-Chi

Tsai, Wei-Lun

Tsai, Ya Hui
Tsai, Yuan-Chen

Tsakalof, Andreas

Tsatsakis, Aristidis

Tsaur, Igor

Tschernig, Thomas

Tseng, Chun-Chih

Tseng, Jenchih

Tseng, Jen-Chih

Tseng-Rogenski, Stephanie S.

Tsingotjidou, Anastasia

Tsirigotis, Panagiotis

Tsoi, Ho

Tsoli, Maria

Tsougos, Ioannis

Tsuboi, Mayo

Tsubota, Akihito

Tsuchiya, Hiroyuki

Tsuchiya, Tomoshi

Tsuchiya, Yuichi

Tsuji, Atsushi B.

Tsuji, Takahiro

Tsujikawa, Takahiro

Tsukada, Hideo

Tsukahara, Toshifumi

Tsuneoka, Makoto

Tsurusaki, Masakatsu

Tsuzuki, Toyonori

Tucci, Marco

Tucci, Paola

Tucker, Matthew

Tuech, Jean-Jacques

Tulchinsky, Eugene

Tummala, Pavan

Turashvili, Gulisa

Turco, Luigi Carlo

Turcotte, Simon

Turdo, Alice

Turesson, Ingela E.

Turetta, Matteo

Turhan, Ali G.

Turpin, Anthony

Tuynman, Jurriaan

Tvrdik, Petr

Tyagi, Ashish

Tyan, Yu-Chang

Tylkowski, Bartosz

Tysarowski, Andrzej

Tzanakakis, George

Tzang, Bor-Show

Tzankov, Alexandar

Tzelepi, Vasiliki 
Tzeng, I-Shiang

Tziatzios, Georgios

Uchiumi, Fumiaki

Udager, Aaron M.

Uddin, Shahab

Uehara, Keiichiro

Uetake, Hiroyuki

Ugel, Stefano

Ugga, Lorenzo

Ugolini, Clara

Uhl, Eberhard

Ukidve, Anvay

Um, Hong-Duck

Umansky, Victor $Y$.

Umansky, Viktor

Umemori, Juzoh

Umemura, Akira

Umemura, Tsukuru

Ung, Choong Yong

Ungefroren, Hendrik

Ungureanu, Bogdan Silviu

Upadhyaya, Jasbir

Uprety, Dipesh

Urbanek-Trzeciak, Martyna O.

Urbanelli, Lorena

Urbaniak, Alicja

Urbanska, Edyta Maria

Urbanucci, Alfonso

Urbschat, Steffi M.

Ureshino, Hiroshi

Ursic-Bedoya, José

Ursino, Stefano

Urso, Emanuele

Ushmorov, Alexey

Usui, Tatsuya

Uygun, Basak E.

Uyl-de Groot, Carin A.

Uysal-Onganer, Pinar

Uzunoglu, Faik G.

Vacca, Angelo

Vaccari, Samuele

Vachtenheim, Jiri

Vaclavikova, Radka

Vaddi, Prasanna

Vaickus, Louis J.

Valachis, Antonis

Valasoulis, George

Valencia, Karmele

Valente, Guido

Valentijn, L. J.

Valentiner, Ursula
Valentini, Valentino

Valentini, Virginia

Valeryia, Valeryia Mikalayeva

Valiante, Salvatore

Valiveti, Chaitanya

Vallacchi, Viviana

Valle, Marcos Eduardo

Vallera, Daniel

Vallet, Sonia

Valletti, Alessio

Valteau-Couanet, Dominique

Valtorta, Silvia

Valverde, Claudia

Vamvakaris, Ioannis

Van Bokhoven, Adrie

Van Dam, Peter A.

Van Dam, R. Michael

Van De Sande, Michiel A. J.

Van De Ven, Rieneke

Van De Wiele, Christophe

Van Den Bergh, Hubert

Van Den Brekel, Michiel Wilhelmus Maria

Van Den Heuvel, Michel

Van Den Tol, Monique Petrousjka

Van Der Hage, Jos A.

Van Der Meel, Roy

Van Der Post, Rachel S.

Van Der Velden, Jacobus

Van Der Wekken, Anthonie

Van Deurzen, Carolien H. M.

Van Dyk, Madele

Van Gompel, Jamie J.

Van Gool, Stefaan W.

Van Griethuysen, Joost

Van Heetvelde, Mattias

Van Hemelrijck, Mieke

Van Houtte, Paul

Van Kempen, Leon

Van Leeuwen, Frank N.

Van Leeuwen, Ton G.

Van Lith, Sanne

Van Moorselaar, Reindert Jeroen A.

Van Noorden, Cornelis Johannes Forrindinis

Van Praet, Charles

Van Prooijen, Monique

Van Schil, Paul

Van Spriel, Annemiek

Van Tine, Brian

Van Tine, Brian Andrew

Van Veldhuisen, Eran

Van Veldhuizen, Peter 
Van Wely, Karel

Van Werkhoven, Erik

Van Wilpe, Sandra

Van Zandwijk, Nico

Vandamme, Timon

VandenBussche, Christopher J.

Van-Dijk, Juliette

Vangipurapu, Rajanikanth

Vanni, Gianluca

Vannier, Michael

Vannini, Eleonora

Vaquero, Javier

Varadaraj, Archana

Varaljai, Renata

Varamini, Pegah

Varga, Georg

Vargas-Parra, Gardenia

Vargova, Karina

Varkaris, Andreas

Varlotto, John Michael

Vasaikar, Suhas V.

Vasan, Nilesh R.

Vasavada, Bhavin

Vasko, Vasyl V.

Vásquez-Gómez, Jaime

Vassos, Nikolaos

Vasuri, Francesco

Vaubel, Rachael

Vaupel, Peter

Vazana, Udi

Vecchio, Giada Maria

Veenland, Jifke

Vega, Francisco M.

Vega-Benedetti, Ana Florencia

Vegran, Frederique

Vekariya, Rakesh

Velegzhaninov, Ilya O.

Velez-Cruz, Renier

Velikova, Tsvetelina

Velliou, Eirini

Velotti, Nunzio

Venanzi, Mariano

Venditti, Adriano

Venesio, Tiziana

Venkatadri, Rajkumar

Venner, Christopher Paul

Venneti, Sriram

Vens, Conchita

Venturelli, Sascha

Venugopalan, Abhilash

Verderio, Claudia
Verdon, Daniel John

Vered, Marilena

Vergara, Daniele

Vergez, François

Verheij, Joanne

Verheij, Marcel

Verheijen, Rene

Verhoeyen, Els

Verma, Navin Kumar

Verma, Shiv

Verma, Shiv Prakash

Vermassen, Tijl

Vermeij, Wilbert P.

Vermeulen, An

Vermijlen, David

Vermorken, Jan B.

Vernieri, Claudio

Vernuccio, Federica

Veronese, Federica

Veronesi, Paolo

Verrelle, Pierre

Versari, Annibale

Vertegaal, Alfred

Verticchio, Alice

Verwaal, Victor Jilbert

Vesa, Stefan Cristian

Veschi, Serena

Veschi, Veronica

Veskoukis, Aristidis S.

Vestal, Deborah J.

Vestergaard, Lau K.

Vetrano, Ignazio Gaspare

Vezzani, Bianca

Vicario, Nunzio

Vicent, Silvestre

Vider, Jelena

Vidovic, Dejan

Vidy, Aurore

Viel, Alessandra

Viel, Sébastien

Víglaský, Viktor

Vignani, Francesca

Vigneswaran, Wickii Thambiah

Vignoli, Alessia

Vigon, Isabelle

Vijayasubhash, Vinod

Vilgrain, Valérie

Villabona, Lisa

Villagomez-Bernabe, Balder

Villa-Morales, María

Villaverde, Gonzalo 
Villegas, Victoria

Vincent-Salomon, Anne

Vincenzo, Rocchetti

Vinci, Maria

Vinciguerra, Manlio

Vinti, Luciana

Viola, Giampietro

Violet, Pierre Christian

Viossat, Yannick

Virolle, Thierry

Virumbrales-Muñoz, María

Visani, Giuseppe

Visconti, Roberto Moro

Visentin, Fabiano

Visone, Rosa

Visser, Lydia

Vitale, Alessandro

Vitale, Chiara

Vitale, Giovanni

Vitale, Mario

Vitale, Rosa Maria

Viti, Vincenza

Vito, Alyssa

Viúdez, Antonio

Vivas Mejia, Pablo

Vizler, Csaba

Vizovišek, Matej

Vlahopoulos, Spiros

Vlašić, Ignacija

Vlassi, Metaxia

Vleminckx, Kris

Vögeli, Thomas-Alexander

Voisset, Edwige

Vojtek, Martin

Volinia, Stefano

Volkmer, Beate

Von Behren, Julie

Von Bueren, André O.

Von Der Grün, Jens

Von Eggeling, Ferdinand

Von Eyben, Finn Edler

Von Neuhoff, Nils

Vošmik, Milan

Voulgarelis, Michael

Voutsinas, Gerassimos E.

Vozenin, Marie-Catherine

Vraka, Chrysoula

Vranic, Semir

Vrettos, Nicholas

Vriend, Jerry

Vujanovic, Nikola
Vymetalkova, Veronika

Wada, Koichiro

Wade, Ros

Wadell, Göran

Wadham, Carol

Wagner, Arthur

Wagner, Kay-Dietrich

Wagner, Kay-Uwe

Wagner, Lars

Wagner, Michael J.

Wagner, Richard

Wagner, Steffen

Wagner, Tristan

Wagner, Waldemar

Wainman, Alan

Wakabayashi, Yuichi

Wakatsuki, Masaru

Wakimoto, Hiroaki

Wałajtys-Rode, Elżbieta

Walczyk, Agnieszka

Wald, Abigail I.

Waldron, Richard T.

Walker, Brian

Walker, Paul Raymond

Wallin, Göran

Walsh, Naomi

Walter, Franziska

Walter, Ingrid

Wan, Thomas

Wang, Cheng-Hsu

Wang, Cheng-Yi

Wang, Chihyang

Wang, Chun

Wang, Dejie

Wang, Guannan

Wang, Guohao

Wang, Hanwen

Wang, Hee-Jung

Wang, Herui

Wang, Hesheng

Wang, Hsiang-Chen

Wang, Huaishan

Wang, Huanyou

Wang, Jeffrey

Wang, Jiao

Wang, Jiaxing

Wang, Jing-Quan

Wang, Junfeng

Wang, Kai

Wang, Liang

Wang, Lily Hui-Ching 
Wang, Lisheng

Wang, Lu

Wang, Po-Hui

Wang, Shidan

Wang, Ting

Wang, Wei

Wang, Yan-Hsiung

Wang, Yuan-Hsi

Wang, Yu-Chih

Wanibuchi, Hideki

Waqar, Mueez

Ward, Douglas

Ward, Stephen C.

Warnakulasuriya, Saman

Warnken, Zachary

Watabe, Tadashi

Watanabe, Eiichi

Watanabe, Yoichi

Watanabe, Yutaka

Watek, Marzena

Waterfall, Joshua J.

Waterton, John

Watson, Steve

Watts, Caroline

Webber, Kate

Weber, Klaus

Weber, Thomas

Weber, Wolfgang

Webster, Marie

Wei, Hsiu-Chuan

Wei, Jun

Wei, Jun S.

Wei, Lei

Wei, Yonglong

Weichhaus, Michael

Weinfeld, Michael

Weis, Serge

Weishaupt, Carsten

Weiss, Noel

Weiss, Robert

Wei-Wu Chen, Tom

Welch, John S.

Weldon, John

Welling, D. Bradley

Wellner, Ulrich Friedrich

Welsh, Michael

Wendt, Michael

Wenger, Katharina

Weniger, Maximilian

Werner, Andreas

Wertz, Philip W.
Wesołowska, Olga

Wessely, Anja

Westermarck, Jukka

Westphal, Dana

Whelan, Rebecca J.

Whitehall, Vicki

Wibmer, Andreas G.

Wickenhauser, Claudia

Widłak, Piotr

Wieczfińska, Joanna

Wiedenmann, Bertram

Wiegering, Verena

Wiemer, Erik A. C.

Wierzejska, Regina

Wiesenfeld, David

Wieser, Rotraud

Wiesner, Georgia L.

Wiggermann, Philipp

Wijaya, Ramesh

Wiktorska, Katarzyna

Wilczek, Brigitte

Wilczyński, Jacek R.

Wilhelm, Imola

Wilhelmi, Markus

Wilke, Christopher T.

Wilkens, Ludwig

Wilkins, Simon

Willey, Christopher

William, Tracy A.

William, Vermi

Williams, Graeme P.

Williams, Norman R.

Wilm, Matthias

Wilmink, Hanneke

Winer, Ira S.

Winham, Stacey J.

Winn, Deborah M.

Winter, Christof

Winter, Gordon

Winter, Stefan

Wirscheim, Ingrid

Wirsdörfer, Florian

Wirth, Matthias

Wirth, Ulrich

Witalisz-Siepracka, Agnieszka

Witarski, Wojciech

Witek, Malgorzata A.

Witkowska Pilaszewicz, Olga

Witte, Hanno M.

Wlodawer, Alexander

Woessmann, Wilhelm 
Woitek, Ramona

Wojewoda, Marta

Wolach, Ofir

Wolf, Benjamin

Wolf, Katarina

Wollmann, Guido

Wołowiec, Dariusz

Won, Misun

Wong, Chi Chun

Wong, Chi-Ming

Wong, Kwan Yeung

Woo, Chang-Hoon

Woo, Shiao

Woo, Sungmin

Wood, Kevin

Wood, Lori Anne

Woods, Amina S.

Woodworth, Graeme F.

Worni, Mathias

Worst, Thomas Stefan

Woźniak, Marta

Wozny, Anne-Sophie

Wren, Dörte

Wright, Roni Helene Grace

Wrobel, Tomasz P.

Wróblewska, Joanna

Wrotek, Sylwia

$\mathrm{Wu}$, Alexander T. H.

$\mathrm{Wu}$, Anna H.

$\mathrm{Wu}$, Cen

$\mathrm{Wu}$, Chengyue

Wu, Chiao-En

Wu, Chun-Te

Wu, Chunyi

$\mathrm{Wu}, \mathrm{Di}$

$\mathrm{Wu}$, Jianming

$\mathrm{Wu}$, Licun

$\mathrm{Wu}$, Ming-Hsun

$\mathrm{Wu}$, Ming-Jiuan

$\mathrm{Wu}$, Rigumula

$\mathrm{Wu}$, Ting

$\mathrm{Wu}$, William

$\mathrm{Wu}$, Xiaohua

Wu, Xuedan

$\mathrm{Wu}$, Yi Hui

$\mathrm{Wu}$, Youjun

$\mathrm{Wu}$, Yuan Hua

$\mathrm{Wu}, \mathrm{Yu}$-shan

Wullaert, Andy

Xavier, Sastre-Garau

Xia, Bing
Xia, Jun

Xiao, Hua

Xiao, Zhousheng

Xie, Changqing

Xie, Guofeng

Xie, Xian Jin

Xie, Zhiqun

Xie, Zhongqiu

Xie, Zuoxu

$\mathrm{Xu}$, Meifeng

$\mathrm{Xu}$, Song-Hui

$\mathrm{Xu}$, Xiaoyin

$\mathrm{Xu}$, Xuehua

$\mathrm{Xu}$, Yan

Xue, Feng

Yabe, Mariko

Yadav, Dhananjay

Yadav, Poonam

Yadav, Sushma

Yagi, Hiroshi

Yahia, Massinissa

Yam, Jason C.

Yamada, Hidetaka

Yamada, Suguru

Yamada, Taketo

Yamakawa, Daishi

Yamamoto, Masahiro

Yamamoto, Naoki

Yamamoto, Yutaka

Yamamoto-Ibusuki, Mutsuko

Yamamura, Soichiro

Yamamura, Takeshi

Yamanaka, Ryuya

Yamao, Kentarou

Yamaoka, Toshimitsu

Yamasaki, Satoshi

Yamasaki, Takahiro

Yamashita, Hiroko

Yamashita, Keishi

Yamatsu, Koji

Yamauchi, Akira

Yamazaki, Taiga

Yammani, Raghunatha R.

Yan, Chunhong

Yan, Shengmin

Yan, Yuanwei

Yanagi, Teruki

Yáñez-Mo, María

Yang, Changwon

Yang, Chih-Jen

Yang, Donghan 
Yang, Eun Joo

Yang, Haifeng

Yang, Haitang

Yang, Howard $\mathrm{H}$.

Yang, Hsin-Yi

Yang, Jiann-Jou

Yang, Jiuling

Yang, Jun

Yang, Kai

Yang, Lee-Wei

Yang, Ming-Hsin

Yang, Quansan

Yang, Sejung

Yang, Seung-Ho

Yang, Shengyu

Yang, Sherry X.

Yang, Shun-Fa

Yang, Wei-hsiung

Yang, Wen-Bin

Yang, Yaohua

Yang, Ying

Yang, Yongkang

Yang, Zeng-Quan

Yang, Zhaogang

Yang, Zhiyu Amy

Yang-Hartwich, Yang

Yanguas-Casás, Natalia

Yao, Ryoji

Yao, Xiaolan

Yaoi, Takeshi

Yarravarapu, Nageswari

Yates, Luke A.

Ye, Jing Christine

Ye, Yunpeng

Ye, Zhiwei

Yeager, Nicholas

Yegorov, Yegor

Yeh, Chun-Nan

Yeh, I-Ju

Yeh, Kun-yun

Yeh, Ta-sen

Yélamos, José

Yeong, Joe Poh Sheng

Yeung, Tsz-Lun

Yevsa, Tetyana

Yi, Hee-Gyeong

Yi, Joo Mi

Yi, Tao

Yin, Kanhua

Ying, Michael

Ylösmäki, Erkko
Yochum, Gregory S.

Yoda, Satoshi

Yoh, Tomoaki

Yohe, Marielle E.

Yokobori, Takehiko

Yokomichi, Naosuke

Yokota, Hiroki

Yokota, Tomoya

Yoneda, Yukio

Yong, WeiPeng

Yoo, Jeongsoo

Yoo, So Young

Yoon, Donghoon

Yoon, Hyonok

Yoon, Wonsuck

Yoon, Won-Sup

Yoshida, Daisuke

Yoshida, Masahito

Yoshida, Noriaki

Yoshie, Mikihiro

Yoshii, Yukie

Yoshinami, Tetsuhiro

Yoshino, Hironori

Yoshino, Kiyoshi

Yoshio, Sachiyo

Yoshioka, Ken-ichi

Yoshioka, Kentaro

Yost, Kathleen J.

You, Xiaona

Youn, Hyun Jo

Younes, Sheren

Young, Christian D.

Young, Richard J.

Yousefi, Bardia

Yousefi, Behrooz Hooshyar

Yousefzadeh, Matthew

$\mathrm{Yu}$, Cheng-Ping

$\mathrm{Yu}$, Ching-Fang

$\mathrm{Yu}$, Fangyan

Yu, Herbert

$\mathrm{Yu}$, Huang-Ping

$\mathrm{Yu}$, Jian Q.

Yu, Jianhua

Yu, Jun

$\mathrm{Yu}$, Kai

$\mathrm{Yu}, \mathrm{L}$.

Yu, Ling

$\mathrm{Yu}$, Xiuping

Yu, Yanlin

Yu, Yanping

Yu, Yung-Luen 
Yuasa, Mari

Yue, Junming

Yuge, Ryo

Yun, Seok Joong

Yunes, José Andrés

Yunlong, Chen

Zaboronok, Alexander

Zabucchi, Giuliano

Zacchia, Miriam

Zachos, George

Zachos, Ioannis

Zachou, Kalliopi

Zaed, Ismail

Zagożdżon, Radosław

Zahid, Maliha

Zahnreich, Sebastian

Zaidi, Syed H. E.

Zaika, Alexander

Zaiss, Dietmar

Zaitone, Sawsan A.

Zakaria, Rasheed

Zakharenko, Alexandra

Zakopoulou, Roubini

Zakrzewska, Malgorzata

Zalatnai, Attila

Zambelli, Alberto

Zambello, Renato

Zamboglou, Constantinos

Zambon, Alfonso

Zambrano, Nicola

Zamfir, Carmen Lăcrămioara

Zamò, Alberto

Zan, Hong

Zanelli, Magda

Zanetti, Rossella

Zanetti-Domingues, Laura C.

Zani, Bianca Maria

Zannoni, Gian Franco

Zanzotto, Fabio Massimo

Zapico, Sara Casado

Zaporozhchenko, Ivan A.

Zapotoczny, Gregory

Zaraca, Francesco

Zarnegar, Rasa

Zarobkiewicz, Michał

Zarovni, Natasa

Zarrabi, Kevin

Zarrine-Afsar, Arash

Zattarin, Emma

Zavattari, Patrizia

Zavattaro, Elisa
Zawacka-Pankau, Joanna

Zebisch, Armin

Zedenius, Jan

Zeglis, Brian

Zehbe, Ingeborg

Zehentmayr, Franz

Zeimet, Alain G.

Zeinali, Mina

Zelinka, Tomas

Zemanova, Zuzana

Zemljic-Jokhadar, Spela

Zemski-Berry, Karin A.

Zeng, Bijun

Zeng, Jialiu

Zeng, Yongji

Żeromski, Jan

Zhaler, Stefan

Zhan, Fenghuang

Zhang, Casper J. P.

Zhang, Chengcheng

Zhang, Daniel Xin

Zhang, Dong

Zhang, Ershuai

Zhang, Guangjun

Zhang, Honghai

Zhang, Huan

Zhang, Ji

Zhang, Jialing

Zhang, Jianmin

Zhang, Jilei

Zhang, Jin

Zhang, Jing

Zhang, Jixiang

Zhang, Li

Zhang, Ling

Zhang, $\mathrm{Nu}$

Zhang, Pengfei

Zhang, Qing

Zhang, Xiaotao

Zhang, Xiaowen

Zhang, Xiaoyu

Zhang, Yanfei

Zhang, Yibin

Zhang, Yu-Dong

Zhang, Yun

Zhang, Zengdi

Zhang, Zhiguo

Zhao, Bailin

Zhao, Dan

Zhao, Linbo

Zhao, Weixing 
Zhao, Xianda

Zhao, Yongfeng

Zhao, Yue

Zhen, David

Zheng, Wenxin

Zhong, Xiaobo

Zhou, Chengji

Zhou, Gang

Zhou, Jian

Zhou, Jingying

Zhou, Qingyu

Zhou, Tianhao (Vincent)

Zhou, Weihua

Zhou, Wen

Zhovannik, Ivan

Zhu, Chris

Zhu, Dandan

Zhu, Quing

Zielinska, Paulina

Zielke, Andreas

Zielonke, Nadine

Zifarelli, Giovanni

Zimmer, Jacques

Zimpfer, Annette

Zimta, Alina-Andreea
Zingman, Leonid V.

Zironi, Isabella

Zivny, Jan

Zofall, Martin

Zoia, Cesare

Zojer, Niklas

Zong, Wei-Xing

Zonta, Giulia

Zottel, Alja

Zou, Wen-Quan

Zrnc, Tomislav A.

Zsiros, Emese

Zsuzsanna, Hevessy

Zubiaga, Ana María

Zubiri, Leyre

Zucchetto, Antonella

Zugaza, José Luis

Zúñiga, Leandro

Zuo, Jianmin

Zupunski, Ljubica

Zwaan, C. Michel

Zwaenepoel, Karen

Zwahlen, Daniel

Zwergel, Clemens

Zylicz, Zbigniew 